\title{
Three Essays on Private Labels, Product Quality and the Retailer's Bargaining Power
}

\author{
by \\ Heng Xu \\ A thesis submitted to the Faculty of Graduate and Postdoctoral Affairs \\ in partial fulfillment of the requirements for the degree of
}

Doctor of Philosophy

in

Economics

Department of Economics

Carleton University

Ottawa, Ontario, Canada

(C)2016, Heng Xu 


\section{Abstract}

The first chapter of this thesis studies the motivations of a retailer to launch a private label product in an environment where consumers do not know the quality of the product before consumption. I show that the launch of the private label product can be a form of brand stretching with the sales of a national brand acting as a bond to ensure that the retailer sells a private label product of high quality. Consequently, the retailer would carry and sell the national brand product even in situations where it would not have done so under perfect information. In addition, I demonstrate that other motivations for launching a private label product, namely to strengthen the retailer's bargaining position and to engage in market segmentation, are not fundamentally affected by the presence of asymmetric information.

In the second chapter, an overlapping-generation model is constructed to investigate the motivations of a retailer to launch a private label product in an environment of asymmetric information about its quality. I focus on the case where the national brand product is too costly to be offered by the retailer under perfect information. I show that, under imperfect information, the retailer may sell a positive amount of the national brand product in every period. This is in contrast to the two-period version of this model in which the national brand is sold in one period only. Thus, this analysis shows that the national brand product can be used as a signal for the quality of the private label product in the long run even though its cost is very high relative to that of the private label product. 
In the final chapter, I use data for a large super-market chain in the Chicago area to test two hypotheses regarding the use of private labels by a retailer that also offers national brands. The first hypothesis is whether private labels enhance the retailer's bargaining power vis-à-vis its manufacturers. The second hypothesis is whether the retailer strategically sets its prices to favor its private labels. I find that private labels indeed enable the retailer to receive lower wholesale prices with its national brand manufacturers. Moreover, private labels lead to an increase in retail prices for the national brands in support of the strategic retail pricing hypothesis. I also use a simulation exercise to evaluate the benefit of private labels to the consumers. 
To my grandparents 


\section{Acknowledgements}

First, I am grateful to my thesis supervisors, Dr. Zhiqi Chen and Dr. Konstantinos Metaxoglou, for their patient guidance, encouragement and advice in this process. I appreciate all their contributions of time and ideas to make my Ph.D. experience productive and stimulating.

I would like to thank my committee members, Dr. Hikmet Gunay, Dr. Derect Ireland and Dr. Aggey Semenov, for their reviews and comments. I also would like to express my sincere thanks to the department's Ph.D. program supervisor, Dr. Patrick Coe, for his continued support all along this process. I am thankful to the department Chair, Dr. Keir Armstrong, for providing me a teaching opportunity for different courses in the department.

I thank Dr. Christopher Gunn, Dr. Lynda Khalaf, Dr. Raúl Razo-Garcia, Dr. Abeer Reza, Dr. Marcel-Cristian Voia, Dr. Christopher Worswick, Dr. Zhihao Yu and Dr. Jiankang Zhang for providing me helps during my job search.

Thanks to Dawn Bjornson, Marge Brooks, Renée Lortie, Sabrina Robineau and Amanda Wright of the Economics department at Carleton University for their numerous assistance.

I would also like to thank my colleagues and friends, Anand Acharya, Dr. Muhammad Ariffin, Samira Hasanzadeh, Dr. Maoqiong Lin, Dr. Zhenjiang Lin, Chaohui Lu, Alexander Maslov, Vincent Paquet, Yang Zou for all the great times that we have shared at Carleton University. 
I must acknowledge Qing Ma. She was always there cheering me up and stood by me through the good times and bad.

Last, but not least, I am deeply thankful to my parents for their love, support, and sacrifices. Without them, this thesis would never have been written. I dedicate this thesis to the memory of my grandparents, whose role in my life was, and remains, immense. 


\section{Table of Contents}

$\begin{array}{lll}\text { Abstract } & \text { ii }\end{array}$

Acknowledgements $\quad$ v

1 Private Labels and Product Quality under Asymmetric Information 1

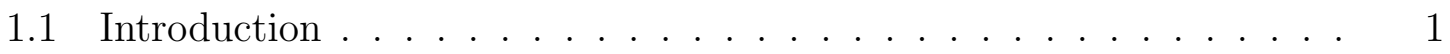

1.2 Model . . . . . . . . . . . . . . . . . . . . . . . . . . 10

1.2 .1 The Firms . . . . . . . . . . . . . . . . . . . . . . . 10

1.2 .2 The Consumers . . . . . . . . . . . . . . . . . . . . . . 12

1.2 .3 The Timing . . . . . . . . . . . . . . . . . . . . . . . 13

1.2.4 The Contract between Firms . . . . . . . . . . . . . 16

1.3 The Equilibrium under Perfect Information: A Benchmark . . . . . 17

1.3.1 The Equilibrium at Stage Three . . . . . . . . . . . . 17

1.3.2 The Equilibrium at Stage Two . . . . . . . . . . . . . . . 23

1.3.3 The Equilibrium at Stage One . . . . . . . . . . . . . . . 23

1.4 Imperfect Information . . . . . . . . . . . . . . . . 26

1.4.1 The Equilibrium with the Retailer's Disagreement Option (0,PL) 27

1.4.2 Case $1(\mathrm{~A}): c_{P L, H}<c_{N B} \leq c_{N B}^{c} \ldots \ldots \ldots \ldots \ldots \ldots$

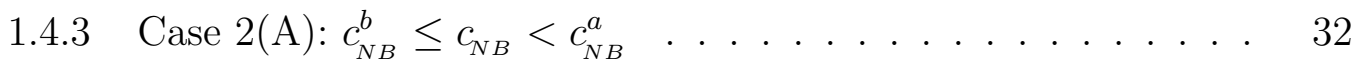

1.4.4 Case $3(\mathrm{~A}): c_{N B}^{c}<c_{N B}<c_{N B}^{b} \ldots \ldots \ldots \ldots \ldots \ldots$

1.5 Consumer Welfare . . . . . . . . . . . . . . . . 42

1.6 Conclusion . . . . . . . . . . . . . . . . . . . . . . . . . . . 43

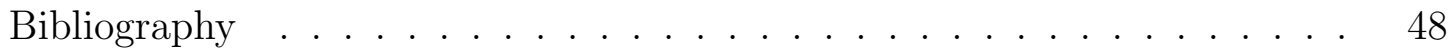

2 Private Labels and Product Quality under Asymmetric Information: $\begin{array}{ll}\text { An Overlapping-Generation Model } & 49\end{array}$

2.1 Introduction . . . . . . . . . . . . . . . . . . . 49

2.2 The Model . . . . . . . . . . . . . . . . . . . . . . . . . . . . . 53 
2.2 .1 The Firms . . . . . . . . . . . . . . . . . . . . . . . . . . . . . . 53

2.2 .2 The Consumers . . . . . . . . . . . . . . . . . . 55

2.2 .3 The Timing . . . . . . . . . . . . . . . . . . . 57

2.2.4 The Contract Between Firms _. . . . . . . . . . . . . 59

2.3 The Equilibrium under Perfect Information: A Benchmark . . . . . . 61

2.3.1 The Equilibrium From Period 2 Onwards . . . . . . . . . . . 61

2.3 .2 The Product Line in Period $2 \ldots \ldots$. . . . . . . . 62

2.3.3 The Equilibrium at Stage Three in Period $1 \ldots \ldots$. . . . . 64

2.3.4 The Equilibrium at Stage Two in Period $1 \ldots \ldots$. . . . . 68

2.3.5 The Equilibrium at Stage One in Period $1 \ldots \ldots$. . . . . . 69

2.4 Imperfect Information . . . . . . . . . . . . . . . 70

2.4.1 The Equilibrium with the Product Line $(0$, PL $) \ldots \ldots .71$

2.4.2 The Equilibrium with the Product Line (NB, PL) . . . . . 75

2.5 Conclusion . . . . . . . . . . . . . . . . . . . 85

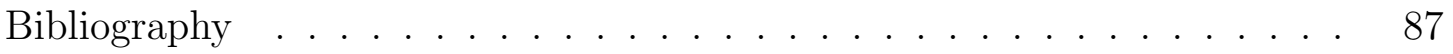

3 Private Labels: Bargaining Power and Strategic Retail Pricing $\quad 88$

3.1 Introduction . . . . . . . . . . . . . . . . . . . . 88

3.2 Model . . . . . . . . . . . . . . . . . . . . . . . . . . . . 93

3.2 .1 Demand Side . . . . . . . . . . . . . . . . . . . . . 93

3.2 .2 Supply Side . . . . . . . . . . . . . . . . . 95

3.3 Estimation . . . . . . . . . . . . . . . . . . . . . . . . . . . 99

3.4 Empirical Analysis . . . . . . . . . . . . . . . . . . . . . . . . 102

3.4 .1 Preliminaries . . . . . . . . . . . . . . . . . . 102

3.4 .2 Demand Estimation . . . . . . . . . . . . . . . . 104

3.4.3 Retailer and Manufacturer Pricing . . . . . . . . . . . 105

3.4.4 Benefits to the Consumers . . . . . . . . . . . . . . . 107

3.5 Conclusion . . . . . . . . . . . . . . . . . . . . . 108

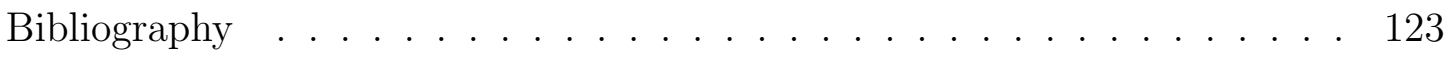

$\begin{array}{ll}\text { A Appendix for Chapter } 1 & 124\end{array}$

B Appendix for Chapter 2 139

$\begin{array}{ll}\text { C Appendix for Chapter 3 } & 149\end{array}$ 


\section{List of Tables}

1.1 Notation . . . . . . . . . . . . . . . . . . . . . . . 17

2.1 The equilibrium profit to the retailer at stage two $\ldots \ldots \ldots$

2.2 The equilibrium profit to the retailer at stage one $\ldots \ldots \ldots$. . . 69

2.3 The equilibrium profit to the retailer at stage two in period $1 \ldots 81$

3.1 National brands . . . . . . . . . . . . . . . . . . . . . . . . 110

3.2 National brands by manufacturer/segment . . . . . . . . . . . . 111

3.3 Private labels . . . . . . . . . . . . . . . . . . . . . . . . . . . 111

3.4 Logit demand estimates: OLS . . . . . . . . . . . . . . . . 112

3.5 Logit demand estimates: IV . . . . . . . . . . . . . . . . . . 113

3.6 Own price elasticities: summary statistics by model . . . . . . . . . 113

3.7 Retailer Pricing Model I . . . . . . . . . . . . . . . . . . . . . . 114

3.8 Retailer Pricing Model II . . . . . . . . . . . . . . . . . . . . 115

3.9 Manufacturer Pricing Model I . . . . . . . . . . . . . . . . . 116

3.10 Manufacturer Pricing Model II . . . . . . . . . . . . . . . . . 117

3.11 Consumer surplus under the baseline and counterfactual scenarios . . 118 


\section{List of Figures}

1.1 The retailer's equilibrium product line under perfect information . . . 8

1.2 The retailers equilibrium product line under imperfect information . . 10

1.3 The vertical relationship among the firms . . . . . . . . . . 11

1.4 The game tree in period $1 \ldots \ldots \ldots \ldots \ldots$

2.1 The retailer's equilibrium product line under imperfect information . 53

2.2 The consumer flow . . . . . . . . . . . . . . . . . 55

3.1 Example of cereal product characteristics . . . . . . . . . . . . . 119

3.2 The Two-stage Model . . . . . . . . . . . . . . . . . . . . . . 120 


\section{Chapter 1}

\section{Private Labels and Product Quality under Asymmetric Information}

\section{$1.1 \quad$ Introduction}

Private labels, also known as store brands, encompass all merchandise sold under a retailer's brand which can be the retailer's own name or a name created exclusively by that retailer. According to a report by the Nielsen Company, private label products accounted for 17.8 percent of the U.S. retailing market by the end of 2011 summer, and 18.9 percent of the one in Canada. These statistics suggest that private labels play a significant role in the market for consumer goods. In Canada, for instance, private label products account for almost one-quarter of total sales in grocery stores, followed by drug stores at around 17 percent. In addition, an increasing number of retailers have moved from selling only national brand products to launching their own private labels. A report from the Agriculture and Agri-Food Canada indicates that, 
although it varies in different regions, the overall trend of private label introduction across the world displays a substantial increase in all analysed regions from 2004 to 2009. ${ }^{1}$ For example, Loblaw's, the largest food retailer in Canada, introduced over 1,500 private label packaged food products from 2008 to 2012 and gained 26.9 percent market share of Canadian private label sales in 2010.

The literature on this subject has studied a number of reasons for a retailer to launch a private label. Some early studies suggest that one of the motivations for introducing a private label is to enrich a retailer's product line. Specifically, in order to better serve heterogeneous consumers, firms may offer intervals of qualities under different brand names to catering to different types of customers. By differentiating the products in one product line with several brands, firms are able to weaken the competition with their rivals so that the corresponding market power is improved (Gilbert and Matutes, 1993). ${ }^{2}$ In marketing, this strategy can be also viewed as market segmentation, with which the firm is able to provide its customers what they want by dividing a broad target market into several subsets. ${ }^{3}$

In respect of the manufacturer-retailer relationship, launching a private label is generally considered as an instrument to strengthen the retailer's bargaining power against the associated national brand supplier (Mills, 1995; Bontems et al., 1999; Bergès-Sennou et al., 2004 for a survey). By launching private label products, a retailer is able to limit the market power of the national brand supplier in a vertical structure and, hence, forces the latter to lower the wholesale price. In other words, when a new substitute is supplied by the retailer and competes with the existing

\footnotetext{
${ }^{1}$ These regions include North America (from 19\% to 22\%), Latin America (from 6\% to 11\%), Europe (from $14 \%$ to $23 \%$ ), Asia Pacific (from $4 \%$ to $7 \%$ ) and Middle East Africa (from $12 \%$ to $22 \%$ ). For more details, see Figure 2 in Agriculture and Agri-Food Canada, Market Analysis Report: Global Private Label Trends.

${ }^{2}$ One seminal study on firms' optimal product line can be found in Champsaur and Rochet (1989).

${ }^{3}$ A seminal study on market segmentation can be found in Smith (1956). For a general survey, see Weinstein (2004), Chapter 1.
} 
good, the effect from the double-marginalization problem would be reduced. It has been shown by Mills (1995) that this effect crucially depends upon the production cost advantage between such product and its branded counterpart. More specifically, if the production costs are identical for both products, the branded product supplier has to lower its wholesale price to compete with private label product as its quality increases. The competition between the national brand and the private label becomes more intensive when both products tend to be less differentiated (with respect to the quality). This weakens the upstream supplier's market power and therefore benefits the retailer. On the other hand, however, if the production costs of the two goods are different (e.g., if the cost on the private label product is increasing and convex in its corresponding quality), improving quality on the private label product would become costly. The retailer's gain from launching a private label may consequently be offset by the increase in production cost. Although the reinforced bargaining power is still possible to the retailer, the condition will tend to be more restrictive (Bontems et al., 1999).

Another branch of studies highlights the impacts of consumers' loyalty on the branded products. Two similar studies, Narasimhan and Wilcox (1998), and Gabrielsen and Sorgard (2007) consider a situation where there are two groups of consumers: one group of consumers is loyal to the national brand, while the other group may switch from the national brand to the private label with a switching cost. ${ }^{4}$ They show that the national brand wholesale price is negatively correlated with the size of the switching group, which indicates that the presence of the switching consumers makes it possible for the retailer to use the threat of launching the private label to enhance is market power. However, as the number of switching consumers falls, the threat of introducing the private label becomes less credible.

\footnotetext{
${ }^{4}$ The latter study assumes that consumers are also differentiated in their tastes.
} 
Moreover, Bergès-Sennou (2006) interprets the characteristics of switching consumers from another angle. When the retailer is allowed to choose whether or not to cooperate with the national brand supplier, a large population of switching consumers may raise its opportunity cost of breaking down the negotiation between the two firms. Namely, if the proportion of the switching consumers is increasing, the retailer prefers to choose a product line contains both national brand and private label and therefore shares the gains with its supplier. As a result, the consumers' loyalty affects not only the retailer's bargaining position, but also the retailer's decision on the composition of its product line.

In addition to the single vertical relation between a manufacturer and a retailer, Avenel and Caprice (2006) as well as Colangelo (2008) investigate the role of private label in a duopolistic downstream market. They all find that the private label products affect the national brand supplier's decision on its selection of dealers. The former study concludes that the supplier prefers to accept all dealers when the cost of national brand product is fairly low. Instead, the latter one sheds light on the degree of substitutability between two private labels. Specifically, the national brand supplier is always in favour of a downstream monopoly when the private labels are less differentiated.

One common feature of the studies cited above is that they are all conducted under the assumption of perfect information. To our knowledge, there has been no study of private labels in an environment of imperfect information where consumers do not know the quality of a private label product before purchase. In reality, however, such information problem may exist, and it may affect the retailer's incentives to launch a private label product. Intuitively, it is easy to see that in a world of asymmetric information, the retailer may have incentive to launch an inferior private label product into the marketplace. This moral hazard problem, in turn, may affect the retailer's 
ability to use the private label to segment the market or to strengthen its bargaining power against the supplier.

The objective of this chapter is therefore to study a retailer's product line decision in an environment where consumers know the quality of a national brand product but do not know the quality of a new private label product. To be more specific, the retailer can offer both the national brand product and the private label product, or offer only one of the two products. The quality of the private label product is determined by the retailer and it can be either high or low. The retailer would not sell a low-quality private label product in a world of perfect information, but it may have incentives to do so in the case where consumers cannot observe the quality before purchase. We will examine the retailer's equilibrium product line as well as the associated retail prices in the presence of this moral hazard problem.

Broadly speaking, this chapter makes two contributions to the literature on private label products. First, we show how the presence of asymmetric information about quality affects the retailer's ability to use a private label product to engage in market segmentation or to strengthen its bargaining power against the supplier of the national brand product. Second, we offer brand stretching as an explanation for a retailer's strategy of carrying both the private label product and the national brand at the same time. Brand stretching, also known as umbrella branding, is a strategy that a firm can use to affect consumers' beliefs about the quality of a new product by marketing it under a well-developed brand. We argue that the launch of private label products by a retailer can be viewed as brand stretching because the private label is uniquely associated with the retailer's brand. In a world of asymmetric information, this gives the retailer another way to signal the quality of its private label product. If it were to sell a private label product of inferior quality, it would ruin its reputation as a retailer and consequently lose the future profits from the sales of the other product 
(i.e., the national brand product). But for this signal to be credible, the retailer has to carry the national brand product even if the profit margin it earns on the national brand product is lower than that on the private label product.

In the literature, Choi (1998) and Cabral (2000) are two prominent studies that examine brand stretching by multi-product firms. Choi (1998) points out that a multiproduct firm can alleviate the problem from asymmetric information by bundling a product of unknown quality with a proven high-quality one. He shows this type of bundling is similar as posting a bond, which is a costly commitment for a low-quality firm since consumers will refuse to purchase again after the low quality is revealed. Similar to Choi (1998), Cabral (2000) uses an adverse selection model to investigate the effects of product qualities on firm's reputation. Specifically, Cabral introduces a feedback reputation effect to characterize the performance of a new product on the sales of base products and shows the performance of such new stretched product positively affects the consumer's further willingness to pay in equilibrium. However, this effect may be weakened if the size of the new product exceeds a certain threshold. This result implies that firm will focus on the sales of the new product when the scale of base product is relatively small. That is, if the new product is relatively profitable compared to the base product, then firms will "exploit" their reputation, so that for a given level of quality, stretching takes place if and only if reputation is sufficiently high. Some similar discussions can also be found in Tadelis (1999), Andersson (2002) and Cabral (2009).

The brand stretching in our model is different from Choi (1998) and Cabral (2000) in that the two products sold by the retailer are of different brands, only one of which is owned by the retailer. One implication of this difference is that the retailer does not have to carry the base product (i.e., the national brand), and our analysis will shed light on the circumstances the retailer does or does not carry the national brand 
product.

The model used in our analysis contains one upstream national brand supplier and one downstream retailer. The latter has the option of launching a private label product by incurring a small but positive sunk cost, and it serves as the threat point in the negotiation between the retailer and the supplier. Products are vertically differentiated, with the national brand product having the highest quality. The firms and the consumers play a game that lasts two periods. To incorporate asymmetric information, we assume that the consumers cannot tell the actual quality of a private label product before the purchase in period 1. However, once the product is sold and consumed in period 1, its quality becomes known to all consumers in period 2, regardless of whether a consumer has actually purchased the product in period 1. Accordingly, consumers who have purchased a high-quality private label product previously will repeat their purchase in period 2. If, on the other hand, the retailer has cheated by selling a low-quality product in period 1, it will lose its reputation completely and consequently, none of the consumers will purchase from this retailer in period 2 .

As a benchmark, we first study the case of perfect information about quality. We show that the retailer's equilibrium product line depends crucially on the relative production costs of the national brand and both high- and low-quality private label products (denoted by $c_{N B}, c_{P L, H}$ and $c_{P L, L}$, respectively). Figure 1.1 below summarizes the equilibriums under perfect information for different ranges of parameter values. If the parameter values fall into region $I$ (i.e., if $c_{N B}$ is relatively low), the retailer will sell only the national brand product in both periods. It will incur the sunk cost needed to launch the private label products, but it will set the price of the latter so high that it will sell zero unit(s) of the private label product in equilibrium. In this case, the private label product is used by the retailer purely as an instrument 
to strengthen its bargaining power against the national brand supplier during the negotiation. On the other hand, if the cost of national brand product is very high relative to that of the private label product (i.e., in region $I I$ ), the retailer will sell the latter only in equilibrium. Finally, if the relative cost of national brand product falls into an intermediate range (i.e., in region $I I I$ ), the retailer will sell both products in equilibrium. Here, the retailer uses the private label product as a tool for market segmentation. Therefore, in the perfect information version of our model, we are able to reproduce the two motivations for private label products, namely to strengthen the retailer's bargaining power and to segment the market.

Figure 1.1: The retailer's equilibrium product line under perfect information

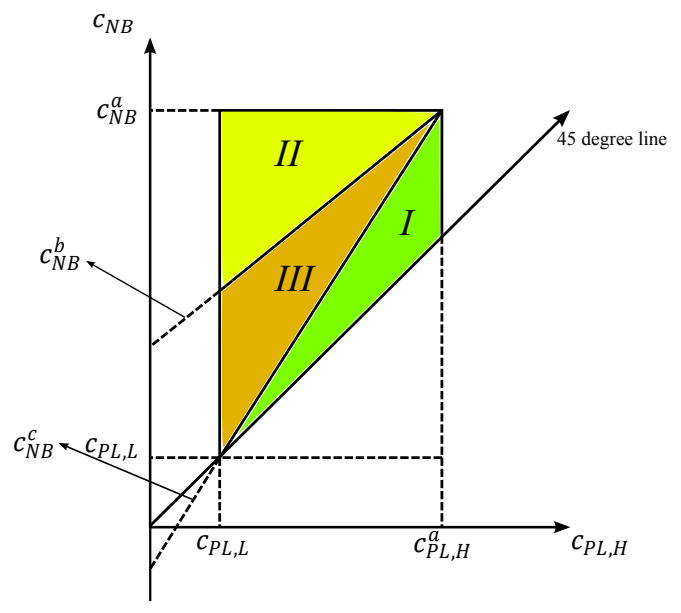

Figure 1.2 summarizes the equilibriums in the case of imperfect information. Of particular interest is sub-region $I V$ that lies in the original region $I I$. If the parameter values fall into sub-region $I V$, the retailer may sell a positive quantity of the national brand product despite the fact that its cost is so high that the retailer would not have sold any units under perfect information. There are two reasons for the presence of this sub-region, both are related to the need for the retailer to credibly signal the quality of its private label product. First, the retailer raises the price of the private 
label product in period 1 (above the equilibrium level under perfect information). The higher price lowers the demand for the private label product and thus reduces the temptation to cheat on quality. The higher price of the private label product also makes it profitable to sell some quantity of the national brand product in period 1. Second, the sale of the national brand product itself becomes a signal of quality in the same way as brand stretching. If the retailer cheats on the quality, it would lose all of customers in period 2 .

Other than the new region in Figure 1.2, the presence of imperfect information does not fundamentally change the retailer's equilibrium product line when the parameter values fall into the other two regions (i.e., the original region $I$ and $I I I$ ) although the imperfect information leads a price distortion on the product with unobserved quality in equilibrium (will be discussed later). Specifically, if the cost of the national brand product is low relative to that of the private label product, introducing a private label (but not actually selling it) is still profitable to the retailer, namely, the retailer can still benefit from using the private label to strengthen its bargaining power against the national brand supplier. ${ }^{5}$ On the other hand, if the cost of the national brand product is neither very high nor very low, market segmentation is attractive to the retailer as usual, but under some circumstances, the retailer will obtain a reduced bargaining profit due to the price distortion from the imperfect information.

The rest of this chapter is organized as follows. Section 1.2 introduces the model. The benchmark case of perfect information is briefly discussed in section 1.3. Section 1.4 solves the model and presents the main results in the case of imperfect information. A brief discussion about consumer welfare is in section 1.5. Section 1.6 concludes.

\footnotetext{
${ }^{5}$ The intuition behind this statement is that by incurring the development cost, the retailer can make a credible threat that it would launch a private label in the event that it fails to reach an agreement with the national brand supplier. The retailer's bargaining power, in turn, is improved with this positive disagreement payoff.
} 
Figure 1.2: The retailers equilibrium product line under imperfect information

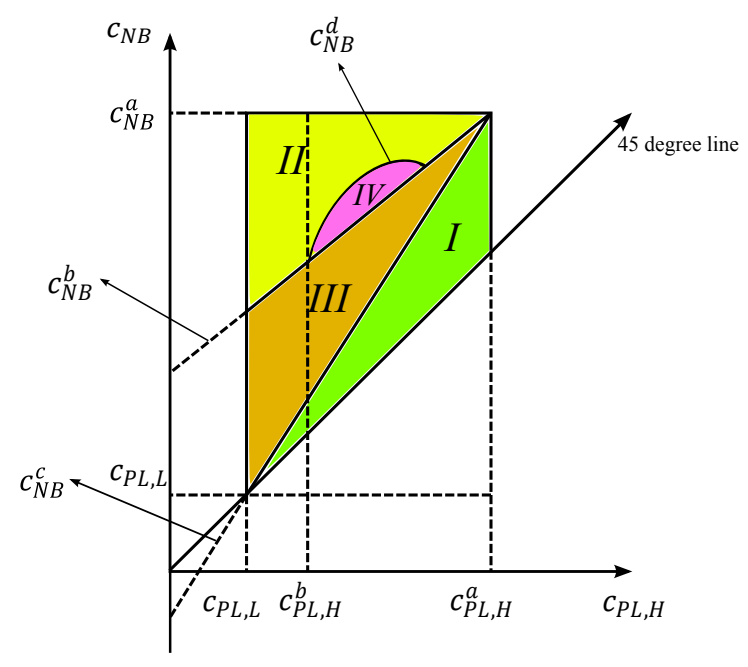

\subsection{Model}

\subsubsection{The Firms}

The model consists of an upstream market and a downstream market. In the upstream market, a monopolistic manufacturer (firm 1) produces a national brand (NB hereafter) consumer product with quality $s_{N B}$ at a constant marginal cost $c_{N B}$. In the downstream market, a retailer (firm 2) buys and resells the NB products at a retail price $p_{N B}$. The retailer, meanwhile, has the option of producing and selling a private label (PL hereafter) product at price $p_{P L}$. The PL product is an imperfect substitute for the NB product in that it has a lower quality. To be more specific, we assume there are two possible levels of quality for the PL product, high $\left(s_{P L, H}\right)$ and low $\left(s_{P L, L}\right)$, with $s_{N B}>s_{P L, H}>s_{P L, L}$. The retailer can produce a high-quality PL product at marginal cost $c_{P L, H}$ or a low-quality PL product at marginal cost $c_{P L, L}$, with $c_{N B}>c_{P L, H}>c_{P L, L}$. Suppose there are no barriers to entry to the production and sales of the low quality product. Accordingly, there are a large number of firms that produce and sell a perfect substitute for the low-quality PL product. In the 
case where the quality of the PL product is known to consumers, the low-quality PL product can only be sold at a price equaling $c_{P L, L}$ due to the competition from these other producers. For ease of discussion, we will refer to this low-quality substitute as the no-name (NN hereafter) product. The vertical relationship among the firms is illustrated in Figure 1.3.

Figure 1.3: The vertical relationship among the firms

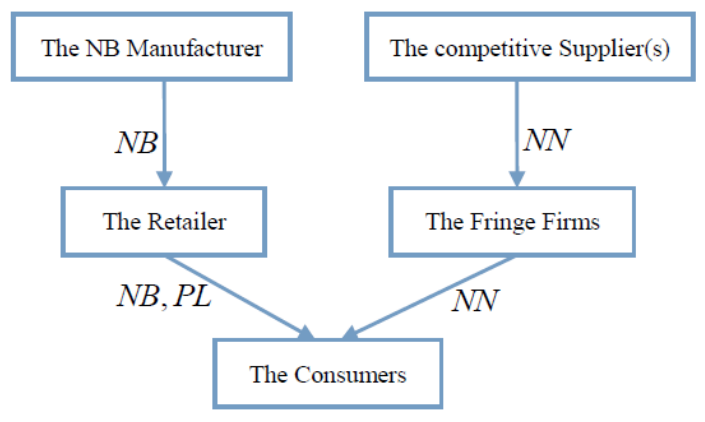

In the event that the retailer chooses to launch the PL product, it has to incur a sunk cost $K$ to design and develop the product. We assume that $K$ is small but positive. This assumption ensures that, in the case where consumers have perfect information about quality, the retailer does not have any incentive to introduce the low-quality private label product since it would not be able to recover the cost $K$.

Suppose that the contract between the supplier and the retailer takes the form of two-part tariff $T=(w, F)$, where $w$ denotes the wholesale price on the NB product and $F$ denotes a fixed fee charged to the retailer. ${ }^{6}$ The contract is determined by the Generalized Nash Bargaining solution. Moreover, we assume that the retailer has to incur a small but positive cost when it enters into a negotiation with the NB supplier. This cost implies that the retailer would prefer the option of producing and selling the PL product if it generates the same payoff as selling the NB product.

\footnotetext{
${ }^{6}$ Since the focus of this chapter is on signalling of product quality, we employ this non-linear price schedule to eliminate the double-marginalization problem.
} 
In term of observability on the product quality, we assume that the quality of the $\mathrm{NB}$ and the NN products is public information while the one of the PL product can be ascertained only after it is consumed by at least some consumers. ${ }^{7}$ The NB supplier, however, is able to find out the quality of the PL product through the negotiation process. This is because in the event that the retailer wants to use the PL product as the threat point in the negotiation process, it will have to present credible evidence to show that it has developed the PL product, and as a professional manufacturer the NB supplier will be able to ascertain the quality of the product from the evidence presented by the retailer.

\subsubsection{The Consumers}

Following the quality-choice model of Mussa and Rosen (1978), we assume that consumers are indexed by their preferences which are uniformly distributed on a vertical interval $[0, \bar{\theta}]$. The distribution has constant density $1 / \bar{\theta}$ over the interval so that the measure is 1 . Consumers at different locations on the interval have different tastes for quality. Specifically, a typical consumer $k$ has a net surplus (indirect utility)

$$
u^{k}=s_{i} \theta^{k}-p_{i}, \quad \theta^{k} \in[0, \bar{\theta}],
$$

if she purchases a product of quality $s_{i}$ at price $p_{i}$, and $u^{k}=0$ if she does not purchase anything. The demand for a product can be derived by determining the location of the marginal consumer who is indifferent between buying this good and the adjacent one. Therefore, the locations of marginal consumers in a two-good market (good $i$ has higher quality) can be determined by equating the net surplus from purchasing

\footnotetext{
${ }^{7}$ Here we exclude the case of common agency, a main reason can be found that is costly for the consumers to purchase the consumer goods through merchandise agents. For more details, see Bernheim and Whinston (1986).
} 
two adjacent products:

$$
\widetilde{\theta}_{i j}=\frac{p_{i}-p_{j}}{s_{i}-s_{j}} \quad \text { and } \quad \widetilde{\theta}_{j 0}=\frac{p_{j}}{s_{j}}
$$

where $\widetilde{\theta}_{i j}$ denotes the location of marginal consumers who are indifferent from buying good $i$ and $j ; \widetilde{\theta}_{j 0}$ denotes the location of marginal consumers who are indifferent from buying good $j$ and nothing. Then the demand for each product is determined by the locations of those marginal consumers as follows:

$$
D_{i}\left(p_{i}, p_{j}\right)=\max \left\{0, \bar{\theta}-\widetilde{\theta}_{i j}\right\} ; \quad D_{j}\left(p_{i}, p_{j}\right)=\max \left\{0, \widetilde{\theta}_{i j}-\widetilde{\theta}_{j 0}\right\}
$$

The demand functions in (1.2) imply that the quantity of either product would be zero if its corresponding retail price is above a certain level. ${ }^{8}$ In addition, because the lower bound of $\theta$ is 0 , there will always be a group of consumers with very low values of $\theta$ who will not purchase even the low quality good at any positive price. In other words, the market will not be fully served in equilibrium.

\subsubsection{The Timing}

Firms and consumers play a game over two periods. The time discount factor is denoted by $\delta \in[0,1]$. The game in the first period has three stages. Specifically, at stage one, the retailer makes a decision on its product line. Because there are two products (i.e., NB and PL) that can be sold through the retailer, the retailer chooses from the following three possible product lines, indexed by $(\mathrm{NB}, 0),(0, \mathrm{PL})$ and (NB, PL). The first two product lines respectively denote the retailer sells only $\mathrm{NB}$ and PL, and the last product line means the retailer sells both products at the same time. The retailer incurs the cost $K$ if it chooses a product line that includes

\footnotetext{
${ }^{8}$ Note that $\frac{\partial D_{i}}{\partial p_{i}}=\frac{\partial D_{i}}{\partial \widetilde{\theta}_{i j}} \cdot \frac{\partial \widetilde{\theta}_{i j}}{\partial p_{i}}<0$, similar to $\frac{\partial D_{j}}{\partial p_{j}}<0$.
} 
the PL product. The retailer's product line remains the same for both periods. In addition, we assume that the NB supplier does not take strategic actions to counter the retailer's use of PL to gain bargaining power. ${ }^{9}$

At stage two, if the retailer has chosen to carry the NB product as part of its product line, it negotiates a contract with the NB supplier that covers both period 1 and period 2. If the two firms fail to reach an agreement, the retailer would sell the PL product only if it has incurred the cost of developing the PL product. Otherwise, its disagreement payoff would be zero. In either case, the disagreement payoff of the NB supplier is zero.

At stage three, the retailer sets the retail prices of the product(s) in period 1 , and consumers make their purchase decisions for period 1. If the retailer carries the PL product, consumers will make their decision based on their belief about its quality. In the case that some consumers purchase the PL product, its quality becomes public knowledge at the end of the first period.

In period 2, the retailer sets the prices for this period, and consumers make their purchase decisions based on their updated belief. More specifically, if the retailer sold the low-quality PL product at an inflated price (i.e., at a price greater than $c_{P L, L}$ ), it will lose all customers in period 2, including those who purchased the NB product in the previous period.

The game tree in Figure 1.4 illustrates the timing of the game in the first period. Note that consumers do not know the quality of the PL product when they observe the product line $(\mathrm{NB}, \mathrm{PL})$ and $(0, \mathrm{PL})$. Parameters $\alpha \in[0,1]$ and $\beta \in[0,1]$ denote the consumers' (first period) prior belief about the quality of the PL product when one of these two product lines are offered. Moreover, when either of these two product

\footnotetext{
${ }^{9}$ The counterstrategies could include widening the quality gap between the NB and the PL, introducing 'fighting brands' to displace PLs on shelves, and launching coupon programmes, etc. (Mills, 1999).
} 
lines is available, a consumer's choice will be "buy" or "not buy" from this retailer depending on her taste. As we have assumed, the consumers who do not buy from this retailer will buy the NN product or nothing. In the case where the retailer offers the product line $(\mathrm{NB}, \mathrm{PL})$, the consumer also makes a decision on which product she wants to buy. Otherwise, the consumer has only one option when she purchases from the retailer. Given our assumption that all the consumers will find out the quality of the PL product once it has been sold in the first period (i.e., their beliefs are updated after some of them have consumed the PL product in the first period), a subset of the consumers will purchase the PL product in period 2 if and only if the retailer has sold a high-quality PL in period 1 . However, all the consumers will refuse to buy from this retailer in period 2 if the low-quality $\mathrm{PL}$ product is detected in the end of period 1.

Figure 1.4: The game tree in period 1

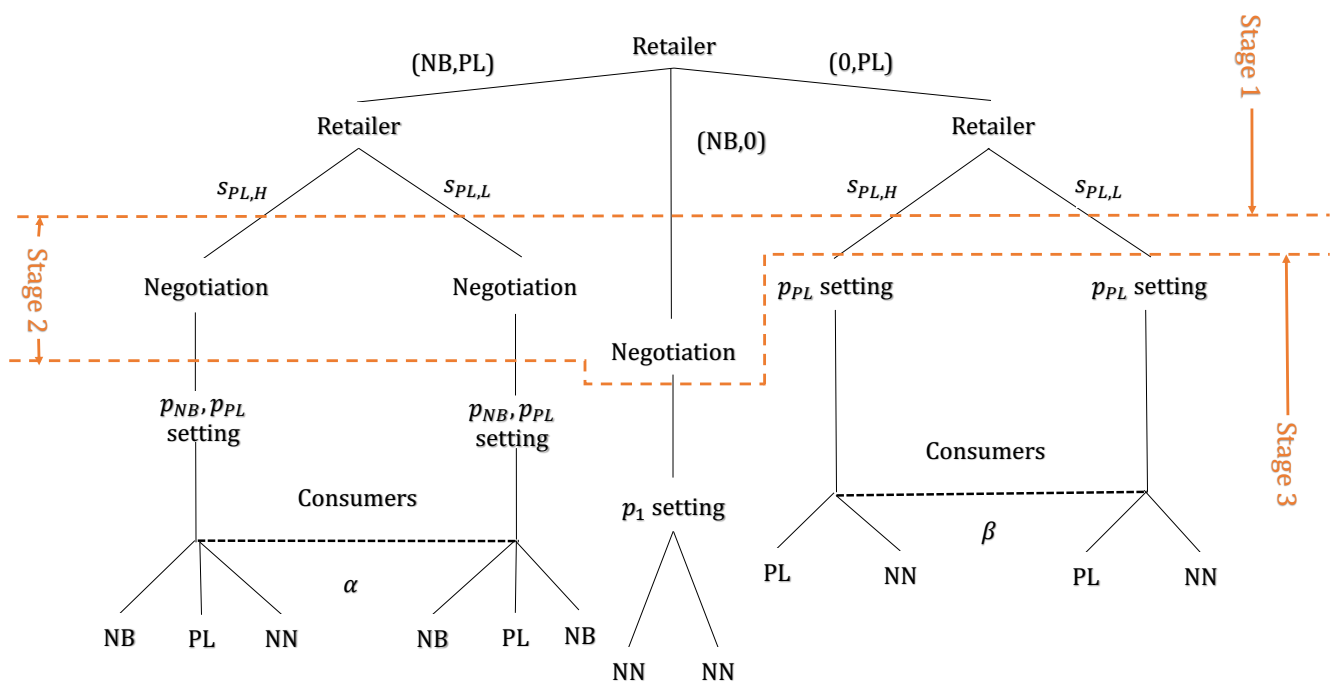




\subsubsection{The Contract between Firms}

Suppose that the terms of contract $T=(w, F)$ between the retailer and the NB supplier are determined by the Generalized Nash Bargaining solution (Harsanyi and Selten, 1972). To be more specific, let $\Pi$ denote the joint profit of the two firms. Then the fixed fee $F$ that the retailer pays to the supplier can be solved from the following Generalized Nash Bargaining problem:

$$
F^{*}=\arg \max _{\pi_{1}}\left(\pi_{1}-\pi_{1}^{0}\right)^{1-\gamma}\left(\pi_{2}-\pi_{2}^{0}\right)^{\gamma} \quad \text { s.t. : } \pi_{1}+\pi_{2}=\Pi,
$$

where $\pi_{i}^{0}$ represents the disagreement payoff to the firm $i$, and $\gamma \in(0,1)$ is a parameter that measures the retailer's bargaining power relative to that of the national brand supplier. This yields the familiar solution:

$$
\pi_{1}^{*}=(1-\gamma) \Pi+\gamma \pi_{1}^{0}-(1-\gamma) \pi_{2}^{0} \quad \text { and } \quad \pi_{2}^{*}=\gamma \Pi-\gamma \pi_{1}^{0}+(1-\gamma) \pi_{2}^{0}
$$

In the special case where both disagreement payoffs are zero, the bargaining power $(\gamma)$ alone determines each firm's share of the joint profit.

With the use of two-part tariff, the contract between the retailer and the supplier is efficient. To be more specific, they will agree on a wholesale price $w$ that maximizes their joint profit. Given the presence of double-marginalization problem (Spengler, 1950), the wholesale price is set to the marginal cost of the national brand product, i.e., $w=c_{N B}{ }^{10}$

All notations mentioned in this section are summarized in the table below.

\footnotetext{
${ }^{10} \mathrm{~A}$ proof to this result can be found in the appendix. For more details about the doublemarginalization problem and nonlinear pricing, see Tirole (1988), chapter 4; Belleflamme and Peitz (2010), chapter 17.
} 
Table 1.1: Notation

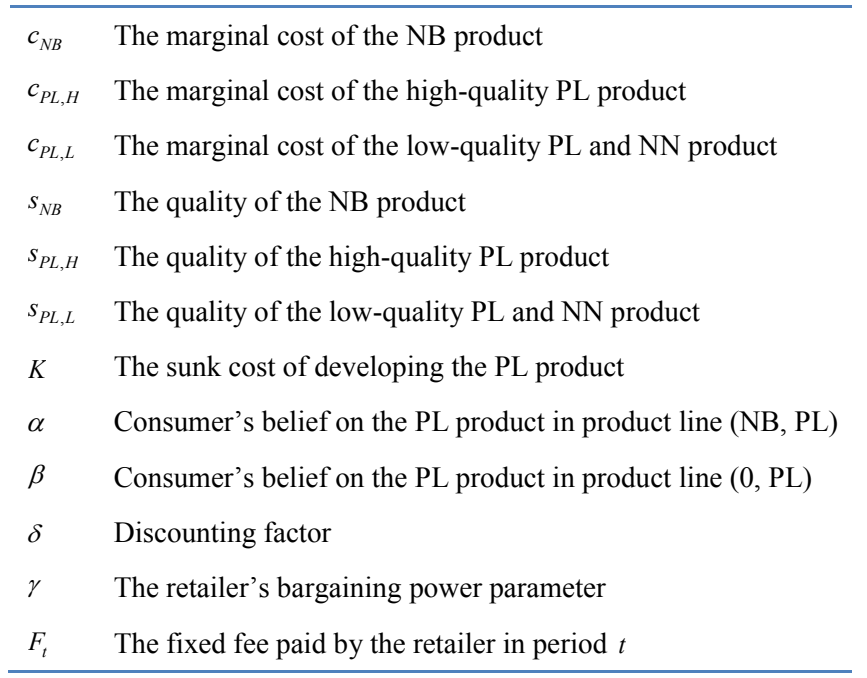

\subsection{The Equilibrium under Perfect Information: A Benchmark}

We first discuss the equilibrium in a perfect information environment where the product quality can be fully observed by all the agents in the market. That is, in Figure 1.4, all information sets at the final stage in the first period are singletons. As we discussed in section 1.2.1, under perfect information, the retailer does not have any incentive to sell low-quality PL since it will obtain a strictly negative profit with the presence of the developing cost $K$. Thus, we can exclude the low-quality PL product from the analysis in this section. In the following sub-sections, we will discuss the equilibriums at different stages of the game.

\subsubsection{The Equilibrium at Stage Three}

Under perfect information, the demand side of the market is identical in the two periods. As a result, the retail price chosen in period 2 is the same as that in period 
1. Accordingly, we need to examine the retailer's choice of price(s) in period 1 only. Since the retailer chooses from three possible product lines, we need to consider three different scenarios.

\subsubsection{The Product Line $(\mathrm{NB}, 0)$}

We start from the sub-game in the middle of the game tree in Figure 1.4. The retailer

only sells the NB product in this sub-game and with the help of (1.2), we can derive the demand function for the NB product

$$
D_{N B}\left(p_{N B}\right)=\max \left\{0, \bar{\theta}-\frac{p_{N B}-c_{N B}}{s_{N B}-s_{P L, L}}\right\}
$$

Thus, the retailer's profit maximization problem is:

$$
\max \Pi\left(p_{N B}\right)=(1+\delta)\left(p_{N B}-c_{N B}\right) D_{N B}\left(p_{N B}\right) .
$$

Solving the above problem yields the equilibrium retail price of NB:

$$
p_{N B}^{*}=\frac{\bar{\theta}}{2}\left(s_{N B}-s_{P L, L}\right)+\frac{c_{N B}}{2}+\frac{c_{P L, L}}{2} .
$$

Substituting the equilibrium price into (1.3), we can derive the equilibrium demand for the NB product as $D_{N B}^{*}=\bar{\theta}-\frac{c_{N B}-c_{P L, L}}{s_{N B}-s_{P L, L}}$. In order to ensure positive demand for the NB product in the product line $(\mathrm{NB}, 0)$, we assume the following

Assumption 1.1 The upper-bound on the marginal cost of the $N B$ product is $c_{N B}^{a}=$ $\bar{\theta}\left(s_{N B}-s_{P L, L}\right)+c_{P L, L}$.

Given Assumption 1.1, we derive the equilibrium joint profit at stage three as:

$$
\Pi_{N B}^{*}=\frac{1+\delta}{4} \frac{1}{s_{N B}-s_{P L, L}}\left(c_{N B}^{a}-c_{N B}\right)^{2} .
$$


One can easily verify that this equilibrium joint profit is convex in $c_{N B}$, and reaches the lowest level (i.e., $\Pi_{N B}^{*}=0$ ) when $c_{N B}$ equals to $c_{N B}^{a}$. Therefore, this equilibrium profit is decreasing in $c_{N B}$ as long as the value of $c_{N B}$ satisfies the Assumption 1.1.

\subsubsection{The Product Line (0,PL)}

The right side of the game tree in Figure 1.4 represents the sub-game where the retailer sells the PL product only. Using (1.2), we find the demand function for the PL product

$$
D_{P L}\left(p_{P L}\right)=\max \left\{0, \bar{\theta}-\frac{p_{P L}-c_{P L, L}}{s_{P L, H}-s_{P L, L}}\right\}
$$

The retailer's profit maximization problem is:

$$
\max \quad \Pi\left(p_{P L}\right)=(1+\delta)\left(p_{P L}-c_{P L, L}\right) D_{P L}\left(p_{P L}\right) .
$$

The solution to this problem is:

$$
p_{P L}^{*}=\frac{\bar{\theta}}{2}\left(s_{P L, H}-s_{P L, L}\right)+\frac{c_{P L, H}}{2}+\frac{c_{P L, L}}{2} .
$$

Substituting the equilibrium price of the PL product into (1.4), we obtain the demand for this good in equilibrium: $D_{P L}^{*}=\bar{\theta}-\frac{c_{P L, H}-c_{P L, L}}{s_{P L, H}-s_{P L, L}}$. In order to ensure positive equilibrium demand for the PL product in the product line $(0, \mathrm{PL})$, we impose the following assumption

Assumption 1.2 The upper-bound on the marginal cost of the PL product is $c_{P L, H}^{a}=$ $\bar{\theta}\left(s_{P L, H}-s_{P L, L}\right)+c_{P L, L}$.

This assumption guarantees that selling (high-quality) PL product can be profitable to the retailer in the case of perfect information. It can be verified that $c_{N B}^{a}-c_{P L, H}^{a}=$ $\bar{\theta}\left(s_{N B}-s_{P L, H}\right)>0$. Thus, Assumptions 1.1 and 1.2 can both be satisfied despite the 
restriction on the relative magnitudes of $c_{N B}$ and $c_{P L, H}$ (namely, $c_{N B}>c_{P L, H}$ ).

Given Assumption 1.2, the retailer's equilibrium profit at stage three is:

$$
\Pi_{P L}^{*}=\frac{1+\delta}{4} \frac{1}{s_{P L, H}-s_{P L, L}}\left(c_{P L, H}^{a}-c_{P L, H}\right)^{2} .
$$

\subsubsection{The Product Line (NB,PL)}

In this sub-game, the retailer carries both products. As we have shown in (1.2), the demand for each good can be expressed as

$$
\begin{gathered}
D_{N B}\left(p_{N B}, p_{P L}\right)=\max \left\{0, \bar{\theta}-\frac{p_{N B}-p_{P L}}{s_{N B}-s_{P L, H}}\right\}, \\
D_{P L}\left(p_{N B}, p_{P L}\right)=\max \left\{0, \frac{p_{N B}-p_{P L}}{s_{N B}-s_{P L, H}}-\frac{p_{P L}-c_{P L, L}}{s_{P L, H}-s_{P L, L}}\right\} .
\end{gathered}
$$

The retailer's profit maximization problem is:

$$
\begin{gathered}
\max \quad \Pi\left(p_{N B}, p_{P L}\right)=(1+\delta)\left[\left(p_{N B}-p_{P L}\right) D_{N B}\left(p_{N B}, p_{P L}\right)+\left(p_{P L}-c_{P L, H}\right) D_{P L}\left(p_{N B}, p_{P L}\right)\right] \\
\text { s.t. }: D_{N B}\left(p_{N B}, p_{P L}\right) \geq 0 ; \quad D_{P L}\left(p_{N B}, p_{P L}\right) \geq 0 .
\end{gathered}
$$

The demand for either good could be zero in this constrained optimization problem. In the case where one of these constraints holds with equality constraint takes place, this optimization problem degenerates into one that has been studied in 1.3.1.1 or 1.3.1.2. If $D_{N B}\left(p_{N B}, p_{P L}\right)=0$, for example, it becomes the one about the product line $(0, \mathrm{PL})$. Similarly, the problem will be consistent with the product line $(\mathrm{NB}, 0)$ when $D_{P L}\left(p_{N B}, p_{P L}\right)=0$.

The Lagrange function associated with this optimization problem is:

$$
\mathscr{L}\left(p_{N B}, p_{P L}, \mu_{1}, \mu_{2}\right)=\Pi\left(p_{N B}, p_{P L}\right)+\mu_{1} D_{N B}\left(p_{N B}, p_{P L}\right)+\mu_{2} D_{P L}\left(p_{N B}, p_{P L}\right) .
$$


The first-order-conditions are:

$$
\begin{aligned}
& \frac{\partial \Pi(\cdot)}{\partial p_{N B}}+\mu_{1} \frac{\partial D_{N B}(\cdot)}{\partial p_{N B}}+\mu_{2} \frac{\partial D_{P L}(\cdot)}{\partial p_{N B}}=0, \\
& \frac{\partial \Pi(\cdot)}{\partial p_{P L}}+\mu_{1} \frac{\partial D_{N B}(\cdot)}{\partial p_{P L}}+\mu_{2} \frac{\partial D_{P L}(\cdot)}{\partial p_{P L}}=0 .
\end{aligned}
$$

If both constraints are non-binding (i.e., if the quantities of both products are positive), both $\mu_{1}$ and $\mu_{2}$ will be zero. By solving (1.7) and (1.8), one can obtain $p_{N B}=p_{N B}^{*}$ and $p_{P L}=p_{P L}^{*}$.

Lemma 1.1 Under perfect information, the equilibrium demands for both products are strictly positive in the product line $(N B, P L)$ if the marginal cost of $N B$ satisfies $c_{N B}^{c}<c_{N B}<c_{N B}^{b}$, where $c_{N B}^{b}=\bar{\theta}\left(s_{N B}-s_{P L, H}\right)+c_{P L, H}$ and $c_{N B}^{c}=\left[c_{P L, H}\left(s_{N B}-s_{P L, L}\right)-\right.$ $\left.c_{P L, L}\left(s_{N B}-s_{P L, H}\right)\right] /\left(s_{P L, H}-s_{P L, L}\right)$. In addition, $c_{P L, H}<c_{N B}^{c}<c_{N B}^{b}<c_{N B}^{a}{ }^{11}$

Lemma 1.1 defines a range of such that the equilibrium quantities of both NB and PL products are all positive. That implies, under perfect information, the retailer wishes to carry both products when the cost of the NB product is neither very high nor very low. Further, when the conditions in Lemma 1.1 hold, one can derive the equilibrium joint profit at stage three by substituting those equilibrium retail prices into the objective function

$$
\Pi_{N B, P L}^{*}=\frac{1+\delta}{4} \frac{1}{s_{N B}-s_{P L, H}}\left[\left(c_{N B}^{a}-c_{N B}\right)\left(c_{N B}^{b}-c_{N B}\right)+\left(c_{P L, H}^{a}-c_{P L, H}\right)\left(c_{N B}-c_{N B}^{c}\right)\right] .
$$

In addition, if one of the constraints is binding, the demand for the corresponding product is zero in equilibrium. In such situation, the equilibrium joint profit at stage three will be identical to the one either from $(\mathrm{NB}, 0)$ or $(0, \mathrm{PL})$. More specifically,

\footnotetext{
${ }^{11}$ The proof of most propositions, lemmas and corollaries in the following discussion can be found in the appendix.
} 
if only the first constraint is binding, then $\mu_{1} \geq 0$ and $\mu_{2}=0$, and the solution to the problem shows $\mu_{1}^{*}=\left(c_{N B}-c_{N B}^{b}\right) / 2$ and $D_{N B}^{*}=0$. And $\mu_{1}^{*} \geq 0$ implies $c_{N B} \geq c_{N B}^{b}$, and $\Pi_{N B, P L}=\Pi_{P L}^{*}$. Similarly, if only the second constraint is binding, then $\mu_{1}=0$ and $\mu_{2} \geq 0$, and the solution to the problem shows $\mu_{2}^{*}=\left(c_{N B}^{c}-c_{N B}\right) /\left(s_{N B}-s_{P L, L}\right)$ and $D_{P L}^{*}=0$. And $\mu_{2}^{*} \geq 0$ entails $c_{N B} \leq c_{N B}^{c}$, and $\Pi_{N B, P L}=\Pi_{N B, P L}^{*}$. To summarize, we have the following three cases that depend on the value of $c_{N B}$ :

- If $c_{P L, H}<c_{N B} \leq c_{N B}^{c}$, the demand for the PL product is zero in product line $(\mathrm{NB}, \mathrm{PL})$, and $\Pi_{N B, P L}=\Pi_{N B}^{*}$;

- If $c_{N B}^{c}<c_{N B}<c_{N B}^{b}$, the demands for both the NB product and the PL product are positive in product line (NB, PL), and $\Pi_{N B, P L}=\Pi_{N B, P L}^{*}$;

- If $c_{N B}^{b} \leq c_{N B}<c_{N B}^{a}$, the demand for the NB product is zero in product line (NB, $\mathrm{PL}$ ), and $\Pi_{N B, P L}=\Pi_{P L}^{*}$.

These three scenarios provide a criterion to the retailer's decision on its product line based on the cost of the NB product under perfect information. If, for example, this cost is neither very high nor very low, the retailer prefers to engage in a market segmentation strategy with the PL product. This strategy, as most analyses discussed, is to address the heterogeneous tastes of the consumers. At the same time, the launch of the PL product also strengthens the retailer's bargaining position at the disagreement point. On the other hand, if producing a unit of the NB product is very cheap relative to the PL product, the retailer may not want to sell a positive amount of the PL product. Instead, it would launch the PL product (by paying the cost $K$ ) solely for the purpose of strengthening its disagreement point. 


\subsubsection{The Equilibrium at Stage Two}

Recall that the negotiation between the retailer and the supplier takes place only if the retailer chooses a product line that contains the NB product. Based on the generalized Nash bargaining solution, the retailer's bargaining payoff with product line $(\mathrm{NB}, 0)$ is $\gamma \Pi_{N B}^{*}$. Given that the retailer has selected "selling NB only" at stage one, it cannot move to a product line that involves the PL product in the event of disagreement. Consequently, the disagreement payoff to both firms is zero and they simply split the joint profit obtained at stage three.

On the other hand, if the product line (NB, PL) has been chosen at stage 1, it means that the retailer has developed its own brand at stage one and has the ability to sell this product in the event of disagreement. This yields a positive disagreement payoff to the retailer as long as the condition in Assumption 1.2 holds. As a result, the retailer's bargaining payoff with product line (NB, PL) is $\gamma \Pi_{N B, P L}+(1-\gamma) \Pi_{P L}^{*}$. Since the value of $\Pi_{N B, P L}$ is different for $c_{N B}$ in different ranges, the retailer's bargaining payoff at stage two depends on the value of $c_{N B}$.

\subsubsection{The Equilibrium at Stage One}

The retailer makes a decision on the product line at this stage. As we have shown in 1.3.1.3, the equilibrium at stage three of the game varies with the value of $c_{N B}$. Accordingly, our analysis in this section has to be conducted for different ranges of $c_{N B}$.

\subsubsection{If $c_{P L, H}<c_{N B} \leq c_{N B}^{c}$}

The value of $c_{N B}$ is very low relative to $c_{P L, H}$ in this case, and hence it is not profitable for the retailer to sell a positive amount of the PL product. But it launches the PL product and carries the product line (NB, PL) solely for the purpose of improving its 
payoff at the disagreement point.

Proposition 1.1 Under complete information, if $c_{P L, H}<c_{N B} \leq c_{N B}^{c}$, the sub-game perfect Nash equilibrium (SPNE) is (NB, PL). In addition, the equilibrium quantity of the PL product is zero.

Proposition 1.1 demonstrates that in the case where the production cost of PL is fairly high relative to the one of $\mathrm{NB}$, the retailer's bargaining power is reinforced by a profitable disagreement option although it does not need to actually sell a positive amount of the PL product in equilibrium. Specifically, once the retailer has developed the store brand at stage one, the information about the existence of a feasible disagreement option would be conveyed to the NB supplier. Due to a strictly positive disagreement payoff, the retailer's bargaining payoff is improved. The development of the PL product makes it credible for the retailer to produce and sell it to consumers in the event of a disagreement with the NB supplier, but in equilibrium there is no disagreement and hence the retailer does not need to sell the PL product.

\subsubsection{If $c_{N B}^{b} \leq c_{N B}<c_{N B}^{a}$}

If the marginal cost of producing the NB product is very high relative to that of the PL product, selling a positive amount of NB becomes unprofitable in a diversified product line (NB, PL). Thus, as we discussed, the joint profit from (NB, PL) will be $\Pi_{P L}^{*}$ in the equilibrium at stage three. The retailer's bargaining payoff (if the retailer actually negotiates with the NB supplier) from such product line is therefore $\gamma \Pi_{P L}^{*}+(1-\gamma) \Pi_{P L}^{*}-K$ or $\Pi_{P L}^{*}-K$, which is exactly same as the one from its disagreement option. In other words, the retailer would gain nothing from negotiating with the NB supplier.

Proposition 1.2 Under complete information, if $c_{N B}^{b} \leq c_{N B}<c_{N B}^{a}$, the SPNE is $(0$, $P L)$. 
Proposition 1.2 first reveals that the retailer will wish to launch and sell its own brand product individually if the marginal cost of the NB product is relatively high. In addition, according to the comparison among the retailer's stage-two equilibrium profits (details can be found in the proof of this proposition), the one from the product line $(0, \mathrm{PL})$ dominates that from $(\mathrm{NB}, 0)$. That implies the retailer does not have incentive to engage in a negotiation with the NB supplier in this case.

\subsubsection{If $c_{N B}^{c}<c_{N B}<c_{N B}^{b}$}

If the value of $c_{N B}$ is neither very large nor very small (e.g., if the condition in Lemma 1.1 holds), consumers will demand a positive amount of each product. This enables the retailer to carry both products in equilibrium, as confirmed by the following proposition.

Proposition 1.3 Under complete information, if $c_{N B}^{c}<c_{N B}<c_{N B}^{b}$, the SPNE is $(N B, P L)$. In addition, the equilibrium quantities on both products are positive.

Proposition 1.3 reveals two motivations for introducing the PL product in the case of the cost of the NB product is neither very high nor very low. First, the retailer benefits from market segmentation. By selling two differentiated products, the retailer can catering to different tastes of the heterogeneous consumers and increase its profit.

Second, the retailer's bargaining position is improved in the presence of the PL product. Different from that in case 1 , here the retailer sells a positive amount of this good in equilibrium. This result is consistent with the finding in the existing literatures (e.g., Mills, 1995; Bontems et al., 1999; Narasimhan and Wilcox, 1998; Gabrielsen and Sorgard, 2007). The benefit to the retailer in the existing studies, however, is reflected through the reduction in a take-it-or-leave-it wholesale price of the NB product. In the present model, on the other hand, the retailer gains through an improvement its disagreement payoff. 


\subsection{Imperfect Information}

In this section, we investigate the equilibriums under imperfect information. As discussed in section 1.3, we assume that once the PL product is sold and consumed in period 1 , there is complete information dissemination about its quality. In other words, the quality becomes known to all the consumers in period $2 .^{12}$ This assumption can be justified by what the marketing science calls the word-of-mouth (WoM) effect, i.e., the passing of information about the quality from person to person. The Amazon.com, for example, provides a platform to its customers for sharing the feedback on its product on-line (also known as electronic word-of-mouth, or eWoM). Chevalier and Mayzlin (2006) demonstrate that the Amazon retailers' profitability is significantly correlated to the customers' reviews. Cabral and Hortaçsu (2010), on the other hand, conduct an empirical study on eBay's reputation mechanism, and find that the weekly sales of a typical eBay seller will drop significantly if he first received negative feedback. In addition, such lower reputation is more likely to drive the seller to exit. This WoM effect can also be used to justify the feedback effect in Cabral (2000) and our assumption that all the consumers will refuse to purchase from the retailer in the second period if an inferior product has been sold in the first period.

The information structure of this game has two implications. First, the consumers may not fully believe the PL product is manufactured in high quality. Consequently, as illustrated in Figure 1.4, we have to consider the consumers' belief about quality in cases where the product line includes the PL product. Second, the retailer may have an incentive to cheat and sell a low-quality PL product as a high-quality one. Accordingly, we need to consider the retailer's incentive compatibility constraint.

\footnotetext{
${ }^{12}$ In an extension of this model, we will study on a situation where there is no information dissemination, i.e., the quality of the PL product is known only to those who actually consumed the product.
} 
This constraint may affect the retailer's equilibrium pricing strategy and generate additional restrictions on the critical values of the marginal cost for which a particular product line arises in equilibrium.

\subsubsection{The Equilibrium with the Retailer's Disagreement Op- tion $(0, \mathrm{PL})$}

When the quality of the PL product cannot be observed by the consumers in the first period, the incentive compatibility constraint may change the equilibrium we have solved in the benchmark case. In this sub-section, we will first discuss the changes from imperfect information on the retailer's threat point, i.e., the product line $(0$, PL). To see this, we construct the retailer's modified profit maximization problem

$$
\begin{gathered}
\max \Pi\left(p_{P L}^{1}, p_{P L}^{2}\right)=\left(p_{P L}^{1}-c_{P L, H}\right) D_{P L}^{1}\left(p_{P L}^{1}\right)+\delta\left(p_{P L}^{2}-c_{P L, H}\right) D_{P L}^{2}\left(p_{P L}^{2}\right) \\
\text { s.t. }: \Pi\left(p_{P L}^{1}, p_{P L}^{2}\right) \geq \widetilde{\Pi}\left(p_{P L}^{1}\right) .
\end{gathered}
$$

where $\widetilde{\Pi}\left(p_{P L}^{1}\right)=\left(p_{P L}^{1}-c_{P L, L}\right)\left(\bar{\theta}-\frac{p_{P L}^{1}-c_{P L, L}}{s_{P L, H}-s_{P L, L}}\right)$ represents the retailer's profit from selling the low-quality product. The profit function $\widetilde{\Pi}(\cdot)$ only involves the sales in the first period because the second period demand for the low-quality product will be zero with complete information dissemination. One difference from the perfect information situation is that the retailer in the present case may want to charge different retail prices through the two periods. Because of informational asymmetry, the retailer probably needs to charge a retail price in period 1 that is different from that of perfect information for the purpose of signaling its quality. But this distortion will disappear in the second period since the consumers will know the actual quality of the PL product. The Lagrange function associated with the above problem can be 
expressed as:

$$
\mathscr{L}\left(p_{P L}^{t}, \lambda\right)=\Pi\left(p_{P L}^{t}\right)+\lambda\left[\Pi\left(p_{P L}^{t}\right)-\widetilde{\Pi}\left(p_{P L}^{1}\right)\right]
$$

Note from the demand function in (1.6) that Assumption 1.1 ensures that the demand on a high-quality PL product is positive in the case where consumers believe that the PL product is of high quality. The first-order conditions and the complementary slackness from the above Lagrange function are:

$$
\begin{gathered}
(1+\lambda) \frac{\partial \Pi\left(p_{P L}^{t}\right)}{\partial p_{P L}^{1}}-\lambda \frac{\partial \widetilde{\Pi}\left(p_{P L}^{1}\right)}{\partial p_{P L}^{1}}=0, \\
(1+\lambda) \frac{\partial \Pi\left(p_{P L}^{t}\right)}{\partial p_{P L}^{2}}=0, \\
\lambda \geq 0 ; \quad \Pi\left(p_{P L}^{t}\right)-\widetilde{\Pi}\left(p_{P L}^{1}\right) \geq 0 ; \quad \lambda\left[\Pi\left(p_{P L}^{t}\right)-\widetilde{\Pi}\left(p_{P L}^{1}\right)\right]=0 .
\end{gathered}
$$

To find out how the equilibrium prices may differ from those under the perfect information, we evaluate the first-order condition in (1.9) and (1.10) at the perfect information retail price level $p_{P L}^{*}$ and find

$$
\left.\frac{\partial \mathscr{L}}{\partial p_{P L}^{1}}\right|_{p_{P L}^{1}=p_{P L}^{*}}=\lambda\left[\frac{c_{P L, H}-c_{P L, L}}{s_{P L, H}-s_{P L, L}}\right] \geq 0 \quad \text { and }\left.\quad \frac{\partial \mathscr{L}}{\partial p_{P L}^{2}}\right|_{P_{P L}^{2}=p_{P L}^{*}}=0
$$

These values imply that given a non-negative marginal effect from the incentive compatibility constraint (i.e., $\lambda \geq 0$ ), the retailer charges a price that is no smaller than the perfect information one in the first period. But the retail price in the second period will be the same as the benchmark price. The latter is expected since the complete dissemination of information means that all the consumers know the actual quality of the PL product in the second period, and information becomes perfect in the second period. 
After solved the equations (1.9) and (1.10), we can obtain

$$
p_{P L}^{1}=p_{P L}^{*}+\frac{\lambda}{2}\left(c_{P L, H}-c_{P L, L}\right) \quad \text { and } \quad p_{P L}^{2}=p_{P L}^{*} .
$$

Clearly, the variance between $p_{P L}^{1}$ and $p_{P L}^{*}$ is given by a non-negative term $\lambda\left(c_{P L, H}-\right.$ $\left.c_{P L, L}\right) / 2$. If the multiplier $\lambda$ is zero, the retail prices will be identical through the two periods, which are same as the benchmark level. For this to arise as an equilibrium, we need the conditions on the marginal cost of producing PL given in the following lemma.

Lemma 1.2 Under imperfect information, the retail prices with the product line (0, $P L)$ are unchanged through the two periods and identical to the perfect information level if $c_{P L, H}<c_{P L, H}^{b}$, where $c_{P L, H}^{b}=\frac{\delta}{2+\delta} \bar{\theta}\left(s_{P L, H}-s_{P L, L}\right)+c_{P L, L}$. In addition, the equilibrium profit from $(0, P L)$ at stage three is also same as the one under perfect information, i.e., $\Pi_{P L}^{*}$. On the other hand, the retail price with the product line (0, $P L)$ is $\hat{p}_{P L}^{1}(\hat{\lambda})=p_{P L}^{*}+\frac{\hat{\lambda}}{2}\left(c_{P L, H}-c_{P L, L}\right)$, and $p_{P L}^{2}=p_{P L}^{*}<\hat{p}_{P L}^{1}(\hat{\lambda})$ if $c_{P L, H}^{b} \leq c_{P L, H}<c_{P L, H}^{a}$. The equilibrium profit from $(0, P L)$ at stage three is $\hat{\Pi}_{P L}=\Pi_{P L}^{*}-\frac{\hat{\lambda}^{2}}{4} \frac{\left(c_{P L, H}-c_{P L, L}\right)^{2}}{s_{P L, H}-s_{P L, L}}$.

Note: The "hat" symbol denotes the equilibrium value under incomplete information; the expression of $\hat{\lambda}$ is provided in the proof of this lemma.

Lemma 1.2 describes the changes in the stage-three equilibrium about the retailer's threat point if the quality of the PL product is unobserved. In effect, the equilibrium depends on the retailer's relative benefit from cheating on the quality. Specifically, if the benefit from selling the low-quality product is small, i.e., $c_{P L, H}-c_{P L, L}<\frac{\delta}{2+\delta} \bar{\theta}\left(s_{P L, H}-s_{P L, L}\right)$, or $c_{P L, H}<c_{P L, H}^{b}$, the incentive compatibility condition is not binding and the retailer is able to charge the same retail prices as in the perfect information benchmark. If the benefit exceeds this threshold, on the other hand, the retailer needs to charge a relatively higher retail price (in the first period) 
in order to signal the quality. The higher retail price lowers the quantity of the PL product sold in the first period. This means that a retailer that cheats by selling a low-quality product as the high-quality one would also sell fewer units, thus reducing the temptation for the retailer to deviate to the low-quality product.

The preceding discussion implies that, in the case where the retail price of the PL product in period 1 is raised to signal quality, the retailer earns a smaller profit from selling the product than under perfect information. This, however, does not mean that the retailer's disagreement payoff would drop to zero.

Lemma $1.3 \hat{\Pi}_{P L}>0$.

Lemma 1.3 implies that the retailer's disagreement payoff is always positive. This is true even if $c_{P L, H}$ exceeds the threshold mentioned in Lemma 1.2. Accordingly, the retailer's bargaining payoff at stage two can always be improved by a proportion $1-\gamma$ of $\Pi_{P L}^{*}$ or $\hat{\Pi}_{P L}$ if the selected product line involves the PL product.

In the following sub-sections, we will investigate the equilibrium under imperfect information in each case that corresponds to the one in section 1.3. To distinguish the two scenarios, we add a letter "A" to the latter.

\subsubsection{Case 1(A): $c_{P L, H}<c_{N B} \leq c_{N B}^{c}$}

Under perfect information, the equilibrium product line in this case is (NB, PL) but the retailer sells zero unit of the PL product. That is, launching the PL product solely plays the role of strengthening the retailer's bargaining position. Lemma 1.2 suggests that as we move to the environment of imperfect information, the retailer has a tendency to raise the price of the PL product. Thus, the retailer would still charge a high retail price on the PL product to make the demand for the PL product to be zero in the stage-three sub-game equilibrium. 
Lemma 1.4 Under incomplete information, if $c_{P L, H}<c_{N B} \leq c_{N B}^{c}$, the equilibrium demand for the PL product is zero in the product line (NB, PL) in both periods.

Because no unit of the PL product is sold in the first period, its quality is not known to consumers in period 2. Thus, the equilibrium demand for the PL product stays at zero in period 2. The equilibrium joint profit from the product line (NB, PL) is therefore identical to the one from $(\mathrm{NB}, 0)$, i.e., $\Pi_{N B}^{*}$.

At stage two, the Generalized Nash Bargaining solution implies that the retailer's bargaining profit from selling the NB product is $\gamma \Pi_{N B}^{*}+(1-\gamma) \Pi_{P L}$, where $\Pi_{P L}=\Pi_{P L}^{*}$ if $c_{P L, L}<c_{P L, H}<c_{P L, H}^{b}$ and $\Pi_{P L}=\hat{\Pi}_{P L}$ if $c_{P L, H}^{b} \leq c_{P L, H}<c_{P L, H}^{a}$. The term $(1-\gamma) \Pi_{P L}$ reflects the fact that the retailer will sell the PL product only if it does not reach agreement with the NB supplier. Although this disagreement payoff is shrunk if $c_{P L, H}^{b} \leq c_{P L, H}<c_{P L, H}^{a}$, the retailer still benefits from the positive disagreement payoff (see Lemma 1.3).

Proposition 1.4 If $c_{P L, H}<c_{N B} \leq c_{N B}^{c}$, the Perfect Bayesian equilibrium (PBE) under imperfect information is characterized by the following.

(i) In the case where $c_{P L, L}<c_{P L, H} \leq c_{P L, H}^{b}$, the product line is (NB, PL) and the retailer sets the prices at $p_{N B}^{1}=p_{N B}^{2}=p_{N B}^{*}$ and $p_{P L}^{1}=p_{P L}^{2}=p_{P L}^{*}$. The belief system is $\{\alpha, \beta\}=\{1,1\}$ if $p_{P L}^{1} \geq p_{P L}^{*}$, and $\{\alpha, \beta\}=\{0,0\}$ if $p_{P L}^{1}<p_{P L}^{*}$. The retailer's equilibrium profit is $\gamma \Pi_{N B}^{*}+(1-\gamma) \Pi_{P L}^{*}-K$ and $D_{P L}^{1}=D_{P L}^{2}=0$.

(ii) In the case where $c_{P L, H}^{b} \leq c_{P L, H}<c_{P L, H}^{a}$, the product line is (NB, PL) and the retailer sets the prices at $p_{N B}^{1}=p_{N B}^{2}=p_{N B}^{*}$ and $p_{P L}^{1}=\hat{p}_{P L}(\hat{\lambda}), p_{P L}^{2}=p_{P L}^{*}$. The belief system is $\{\alpha, \beta\}=\{1,1\}$ if $p_{P L}^{1} \geq \hat{p}_{P L}(\hat{\lambda})$, and $\{\alpha, \beta\}=\{0,0\}$ if $p_{P L}^{1}<\hat{p}_{P L}(\hat{\lambda})$. The retailer's equilibrium profit is $\gamma \Pi_{N B}^{*}+(1-\gamma) \hat{\Pi}_{P L}-K$ and $D_{P L}^{1}=D_{P L}^{2}=0$. 
Proposition 1.4 implies that the retailer's motivation for launching PL when the cost of NB product is relatively small is to improve its payoff at the threat point. As we proved in Lemma 1.2 and 1.3, this disagreement payoff is always positive (given Assumption 1.2) and introducing the PL product therefore helps the retailer to enhance its bargaining position against the NB supplier. Here the role of the PL product is qualitatively the same as the one in case 1. Quantitatively, the retailer earns a smaller profit in equilibrium in the situation that $c_{P L, H}^{b} \leq c_{P L, H}<c_{P L, H}^{a}$ because of the higher retail price on the PL product and thus lower disagreement payoff under imperfect information.

\subsubsection{Case 2(A): $c_{N B}^{b} \leq c_{N B}<c_{N B}^{a}$}

The equilibrium in case 2 shows the retailer does not wish to sell a positive amount of NB products under perfect information because of the high cost of this product. When the information is imperfect, however, this result may be changed. This is because a higher retail price on the PL product (due to imperfect information) can make the demand for the NB product positive. To be more specific, the higher price drives down the demand for the PL product, causing some consumers to switch to the NB product. With this in mind, we examine the possibility of positive demand for the NB product in this equilibrium. A modified profit maximization problem at stage three can be written as:

$$
\begin{aligned}
& \max \Pi\left(p_{N B}^{1}, p_{N B}^{2}, p_{P L}^{1}, p_{P L}^{2}\right)=\left[\left(p_{N B}^{1}-c_{N B}\right) D_{N B}^{1}\left(p_{N B}^{1}, p_{P L}^{1}\right)+\left(p_{P L}^{1}-c_{P L, H}\right) D_{P L}^{1}\left(p_{N B}^{1}, p_{P L}^{1}\right)\right] \\
& +\delta\left[\left(p_{N B}^{2}-c_{N B}\right) D_{N B}^{2}\left(p_{N B}^{2}, p_{P L}^{2}\right)+\left(p_{P L}^{2}-c_{P L, H}\right) D_{P L}^{2}\left(p_{N B}^{2}, p_{P L}^{2}\right)\right] \\
& \text { s.t. : } \quad \Pi\left(p_{N B}^{1}, p_{N B}^{2}, p_{P L}^{1}, p_{P L}^{2}\right) \geq \widetilde{\Pi}\left(\Pi\left(p_{N B}^{1}, p_{P L}^{1}\right) ; \quad D_{N B}^{1}\left(\Pi\left(p_{N B}^{1}, p_{P L}^{1}\right) \geq 0\right. \text {, }\right.
\end{aligned}
$$

where $\widetilde{\Pi}\left(\left(p_{N B}^{1}, p_{P L}^{1}\right)=\left(p_{N B}^{1}-c_{N B}\right) D_{N B}^{1}\left(p_{N B}^{1}, p_{P L}^{1}\right)+\left(p_{P L}^{1}-c_{P L, L}\right) D_{P L}^{1}\left(p_{N B}^{1}, p_{P L}^{1}\right)\right.$ represents the profit from selling the low-quality PL product in the product line (NB, PL). 
Again, the profit function $\widetilde{\Pi}(\cdot)$ is independent of the sales in the second period because the low-quality retailer loses all of its customers in the second period. The second constraint in the retailer's optimization problem is to take into account the possibility of positive demand for the NB product. The Lagrange function associated with the above problem can be written as:

$\mathscr{L}\left(\mathbf{p}_{N B}, \mathbf{p}_{P L}, \lambda_{1}, \lambda_{2}\right)=\Pi\left(\mathbf{p}_{N B}, \mathbf{p}_{P L}\right)+\lambda_{1}\left[\Pi\left(\mathbf{p}_{N B}, \mathbf{p}_{P L}\right)-\widetilde{\Pi}\left(p_{N B}^{1}, p_{P L}^{1}\right)\right]+\lambda_{2} D_{N B}^{1}\left(p_{N B}^{1}, p_{P L}^{1}\right)$,

where $\mathbf{p}_{N B}$ and $\mathbf{p}_{P L}$ respectively denote that the vector of prices on the NB and PL product over the two periods. The first-order conditions with respect to the first period retail prices are:

$$
\begin{aligned}
& \frac{\partial \mathscr{L}(\cdot)}{\partial p_{N B}^{1}}=\left(1+\lambda_{1}\right) \frac{\partial \Pi\left(\mathbf{p}_{N B}, \mathbf{p}_{P L}\right)}{\partial p_{N B}^{1}}-\lambda_{1} \frac{\partial \widetilde{\Pi}\left(p_{N B}^{1}, p_{P L}^{1}\right)}{\partial p_{N B}^{1}}+\lambda_{2} \frac{\partial D_{N B}^{1}\left(p_{N B}^{1}, p_{P L}^{1}\right)}{\partial p_{N B}^{1}}=0 \\
& \frac{\partial \mathscr{L}(\cdot)}{\partial p_{P L}^{1}}=\left(1+\lambda_{1}\right) \frac{\partial \Pi\left(\mathbf{p}_{N B}, \mathbf{p}_{P L}\right)}{\partial p_{P L}^{1}}-\lambda_{1} \frac{\partial \widetilde{\Pi}\left(p_{N B}^{1}, p_{P L}^{1}\right)}{\partial p_{P L}^{1}}+\lambda_{2} \frac{\partial D_{N B}^{1}\left(p_{N B}^{1}, p_{P L}^{1}\right)}{\partial p_{P L}^{1}}=0
\end{aligned}
$$

Moreover, the second period retail prices are identical to the ones under perfect information due to complete information dissemination. Also, the demand for each product in the second period is same to the one in case 2. That is, the equilibrium demand for the NB product is zero because of its high cost.

Lemma 1.5 If $c_{N B}^{b} \leq c_{N B}<c_{N B}^{a}$ and $c_{H}<c_{H}^{b}$, the PBE at stage three under imperfect information consists of the product line $(0, P L)$. On the other hand, if $c_{N B}^{b} \leq c_{N B}<c_{N B}^{d}$ and $c_{P L, H}^{b} \leq c_{P L, H}<c_{P L, H}^{a}$ where $c_{N B}^{d}=c_{N B}^{a}-\frac{\delta}{2} \frac{\left(c_{P L, H}^{a}-c_{P L, H}\right)^{2}}{c_{P L, H}-c_{P L, L}}$, the retailer set the prices at $p_{N B}^{1}=p_{N B}^{2}=p_{N B}^{*}$ and $p_{P L}^{1}=\hat{p}_{P L}\left(\hat{\lambda}_{1}\right), p_{P L}^{2}=p_{P L}^{*}$. The belief system is $\{\alpha, \beta\}=\{1,1\}$ if $p_{P L}^{1} \geq \hat{p}_{P L}\left(\hat{\lambda}_{1}\right)$, and $\{\alpha, \beta\}=\{0,0\}$ if $p_{P L}^{1}<\hat{p}_{P L}\left(\hat{\lambda}_{1}\right) .{ }^{13}$ The equilibrium demand for the $N B$ product is positive in the first period.

\footnotetext{
${ }^{13}$ The values of equilibrium prices are provided in the proof of this Lemma.
} 
Lemma 1.5 provides a quite different result from the corresponding one in case 2. More specifically, when the difference in the marginal costs of producing the NB and PL products (i.e., $c_{N B}-c_{P L, H}$ ) is not fairly large, it is possible that the retailer sells a positive amount of the NB products. There are two reasons for this, both are related to the need for the retailer to credibly signal the quality of its private label product. First, the retailer raises the price of the private label product in period 1 (above the equilibrium level under perfect information). The higher price lowers the demand for the private label product and thus reduces the temptation to cheat on quality. The higher price of the private label product also makes it profitable to sell some quantity of the national brand product in period 1. Second, the sale of the national brand product itself becomes a signal of quality in the same way as brand stretching. If the retailer cheats on the quality, it would lose all of customers in period 2 .

At stage two, the retailer's bargaining profit from the product line (NB, PL) can vary with the different values of $c_{N B}$ and $c_{P L, H}$. Specifically, such profit can be the same as the one in case 2, i.e., $\gamma \Pi_{P L}^{*}+(1-\gamma) \Pi_{P L}^{*}-K=\Pi_{P L}^{*}-K$ if $c_{P L, H}$ is sufficiently small, i.e., if $c_{P L, H}<c_{P L, H}^{b}$ (also, the quantity of the NB product is zero in both periods). On the other hand, if $c_{P L, H}$ exceeds such threshold but $c_{N B}$ is not too high, i.e., if $c_{N B}^{b} \leq c_{N B}<c_{N B}^{d}$ (mentioned in Lemma 1.5), this profit will become $\gamma \hat{\Pi}_{N B, P L}+(1-\gamma) \Pi_{P L}-K .{ }^{14}$ According to Lemma 1.2, if the positive demand for the NB product is possible, the retailer's disagreement payoff will shrink from $\Pi_{P L}^{*}$ to $\hat{\Pi}_{P L}$ (since $c_{P L, H} \geq c_{P L, H}^{b}$ in this situation).

Proposition 1.5 If $c_{N B}^{b} \leq c_{N B}<c_{N B}^{a}$, the PBE under imperfect information is characterized by the following.

(i) In the case where $c_{P L, H}<c_{P L, H}^{b}$, the product line is $(0, P L)$ and the retailer sets the prices at $p_{P L}^{1}=p_{P L}^{2}=p_{P L}^{*}$. The belief system is $\{\alpha, \beta\}=\{1,1\}$ if $p_{P L}^{1} \geq p_{P L}^{*}$,

\footnotetext{
${ }^{14}$ The "hat" on $\Pi_{N B, P L}$ has exactly same meaning as before.
} 
and $\{\alpha, \beta\}=\{0,0\}$ if $p_{P L}^{1}<p_{P L}^{*}$. The retailer's equilibrium profit is $\Pi_{P L}^{*}-K$.

(ii) In the case where $c_{P L, H}^{b} \leq c_{P L, H}<c_{P L, H}^{a}$ and $c_{N B}^{b} \leq c_{N B}<c_{N B}^{d}$, the product line is $(N B, P L)$ and the retailer sets the prices at $p_{N B}^{1}=p_{N B}^{2}=p_{N B}^{*}$ and $p_{P L}^{1}=$ $\hat{p}_{P L}\left(\hat{\lambda}_{1}\right), p_{P L}^{2}=p_{P L}^{*}$. The belief system is $\{\alpha, \beta\}=\{1,1\}$ if $p_{P L}^{1} \geq \hat{p}_{P L}\left(\hat{\lambda}_{1}\right)$, and $\{\alpha, \beta\}=\{0,0\}$ if $p_{P L}^{1}<\hat{p}_{P L}\left(\hat{\lambda}_{1}\right)$. The retailer's equilibrium profit is $\gamma \hat{\Pi}_{N B, P L}+$ $(1-\gamma) \hat{\Pi}_{P L}-K$ and $D_{N B}^{1}>0, D_{N B}^{2}=0$.

(iii) In the case where $c_{P L, H}^{b} \leq c_{P L, H}<c_{P L, H}^{a}$ and $c_{N B}^{d} \leq c_{N B}<c_{N B}^{a}$, the product line is $(0, P L)$ and the retailer sets the prices at $p_{P L}^{1}=\hat{p}_{P L}(\hat{\lambda}), p_{P L}^{2}=p_{P L}^{*}$. The belief system is $\{\alpha, \beta\}=\{1,1\}$ if $p_{P L}^{1} \geq \hat{p}_{P L}(\hat{\lambda})$, and $\{\alpha, \beta\}=\{0,0\}$ if $p_{P L}^{1}<\hat{p}_{P L}(\hat{\lambda})$. The retailer's equilibrium profit is $\hat{\Pi}_{P L}-K$.

Proposition 1.5 provides a possibility of diversified equilibrium product line if $c_{P L, H}$ is not sufficiently small, i.e., if $c_{P L, H}^{b} \leq c_{P L, H}<c_{P L, H}^{a}$. It can be verified that the retailer's bargaining profit from the product line $(\mathrm{NB}, \mathrm{PL})$ is greater than that from both $(\mathrm{NB}, 0)$ and $(0, \mathrm{PL})$ if $c_{N B}$ reaches the lowest level (i.e., if $c_{N B}=c_{N B}^{b}$ ) in this case. In addition, the difference between the payoff from (NB, PL) and (NB, 0 ) is increasing in $c_{N B} \in\left[c_{N B}^{b}, c_{N B}^{d}\right)$, whereas the one between the payoff from (NB, $\mathrm{PL})$ and $(0, \mathrm{PL})$ is decreasing in $c_{N B} \in\left[c_{N B}^{b}, c_{N B}^{d}\right)$. The intuition behind this fact is straightforward. While $c_{N B}$ negatively affects the profit from both (NB, PL) and (NB, 0 ), its impact is smaller on the former than on the latter. Thus, the retailer is more willing to abandon the latter as the NB product becomes more costly. On the other hand, the retailer's profit from carrying the product line $(0, P L)$ is independent of $c_{N B}$. As a consequence, the value of $\hat{\Pi}_{N B}-\hat{\Pi}_{P L}$ will be decreasing in $c_{N B} \in\left[c_{N B}^{b}, c_{N B}^{d}\right)$. However, this difference is positive for all $c_{N B} \in\left[c_{N B}^{b}, c_{N B}^{d}\right)$. Therefore, the retailer will always prefer to sell a positive amount of the NB products if the parameter values meet the conditions in Lemma 1.5. 
On the other hand, if $c_{P L, H}$ is smaller than the critical value $c_{P L, H}^{b}$, the retailer will charge a retail prices on the NB product that causes its equilibrium demand in both periods to be zero. In such a case, the equilibrium outcome is qualitatively the same as the one in case 2. On the other hand, if $c_{P L, H}$ still satisfies $c_{P L, H}^{b} \leq c_{P L, H}<c_{P L, H}^{a}$ but the value of $c_{N B}$ is such that $c_{N B}^{d} \leq c_{N B}<c_{N B}^{a}$, there is no PBE with high-quality PL product in $(\mathrm{NB}, \mathrm{PL})$. The retailer therefore makes decision between the product line $(\mathrm{NB}, 0)$ and $(0, \mathrm{PL})$. It can be shown the product line $(\mathrm{NB}, 0)$ is dominated by $(0, \mathrm{PL})$ because the cost of the NB product is too high in this case. Accordingly, the retailer will choose to sell only the PL product.

As a summary, if the difference between the marginal costs of producing the NB and PL product is not sufficiently large, the retailer will prefer to sell both products at the same time (in the first period). In the presence of asymmetric information, the retailer uses the NB product as a credible signal on the unobservable quality of the PL product. In particular, the consumers are more likely to be convinced of the high-quality PL product when the NB product is also sold because the retailer will face a larger punishment of cheating. To be more specific, we can write down the respective punishments to the retailer of cheating in the product line $(0, \mathrm{PL})$ and $(\mathrm{NB}, \mathrm{PL})$ :

$$
\begin{aligned}
P_{P L} & =\hat{\Pi}_{P L}-\widetilde{\Pi}_{P L} \\
& =\delta\left(p_{P L}^{*}-c_{P L, H}\right) D_{P L}^{*}\left(p_{P L}^{*}\right)-\left(c_{P L, H}-c_{P L, L}\right)\left(\bar{\theta}-\frac{\hat{p}_{P L}(\hat{\lambda})-c_{P L, L}}{s_{P L, H}-s_{P L, L}}\right),
\end{aligned}
$$

and

$$
\begin{aligned}
P_{N B, P L} & =\hat{\Pi}_{N B, P L}-\widetilde{\Pi}_{N B, P L} \\
& =\delta\left(p_{P L}^{*}-c_{P L, H}\right) D_{P L}^{*}\left(p_{P L}^{*}\right)-\left(c_{P L, H}-c_{P L, L}\right)\left(\frac{p_{N B}^{*}-p_{P L}\left(\hat{\lambda}_{1}\right)}{s_{N B}-s_{P L, H}}-\frac{\hat{p}_{P L}\left(\hat{\lambda}_{1}\right)-c_{P L, L}}{s_{P L, H}-s_{P L, L}}\right) .
\end{aligned}
$$


Note that the first term in above punishment functions implies that in both product lines, the retailer with high-quality product will sell only the PL product in period 2 and charge the perfect information price.

Corollary 1.1 Under imperfect information, if $c_{N B}^{b} \leq c_{N B}<c_{N B}^{d}$ and $c_{P L, H}^{b} \leq c_{P L, H}<$ $c_{P L, H}^{a}$, the punishment to the retailer for carrying a low-quality private label product is increased if a positive amount of the NB products is sold in a product line, i.e., $P_{N B, P L}>P_{P L}$

Corollary 1.1 highlights one feature of the brand stretching with which the retailer will receive a relatively larger punishment from cheating on the product quality. Different from the literature on brand stretching (Cabral, 2000), in the present model the signal of quality comes from a different firm (i.e., the NB supplier). If the retailer cheats on the quality of its own brand product, it will lose all the customers including the ones who purchase the NB product. Accordingly, the punishment here is not just the loss of the demand for the products under one brand. Rather, it is the loss of all the products sold by this retailer even though they are of different brands.

Moreover, the presence of the NB product also reduces the price distortion on the PL product under asymmetric information. Specifically, the distortions on the price of the PL product in the product line $(0, \mathrm{PL})$ and $(\mathrm{NB}, \mathrm{PL})$ are respectively:

$$
\rho_{P L}=\hat{p}_{P L}(\hat{\lambda})-p_{P L}^{*}=\frac{\hat{\lambda}}{2}\left(c_{P L, H}-c_{P L, L}\right),
$$

and

$$
\rho_{N B, P L}=\hat{p}_{P L}\left(\hat{\lambda}_{1}\right)-p_{P L}^{*}=\frac{\hat{\lambda}_{1}}{2}\left(c_{P L, H}-c_{P L, L}\right) .
$$

Note that the price on the PL product is distorted in the first period only. The distortion will disappear as the information becomes perfect in the second period. 
Corollary 1.2 Under imperfect information, if $c_{N B}^{b} \leq c_{N B}<c_{N B}^{d}$ and $c_{P L, H}^{b} \leq c_{P L, H}<$ $c_{P L, H}^{a}$, then $\rho_{P L}>\rho_{N B, P L}$.

This corollary implies that brand stretching alleviates the informational problem on the price from asymmetric information. While based on a similar mechanism, our result differs from Choi (1998) in two regards. First, the reduction in the equilibrium retail price arises on the product under a different brand from the base product. Second, the alleviation of price distortion by brand stretching is not from selling the new product under the same brand as the high-quality base product, but from the sales of a product under a different brand.

\subsubsection{Case 3(A): $c_{N B}^{c}<c_{N B}<c_{N B}^{b}$}

If the marginal cost of producing the NB product is neither very high nor very low, both products are sold under perfect information. As we discussed in case 2(A), however, the demand for the PL product in the imperfect information environment is smaller in period 1 because the retailer has to raise its price to signal quality. In the present case, the cost-advantage on the PL product is smaller, which may lead a further decrease in the demand for the PL product. Therefore, we must examine the possibility of zero demand for the PL product here. To do this, we modify the retailer's constraint in its profit maximization problem at stage three as follows:

$$
\begin{aligned}
& \max \Pi\left(p_{N B}^{1}, p_{N B}^{2}, p_{P L}^{1}, p_{P L}^{2}\right)=\left[\left(p_{N B}^{1}-c_{N B}\right) D_{N B}^{1}\left(p_{N B}^{1}, p_{P L}^{1}\right)+\left(p_{P L}^{1}-c_{P L, H}\right) D_{P L}^{1}\left(p_{N B}^{1}, p_{P L}^{1}\right)\right] \\
& +\delta\left[\left(p_{N B}^{2}-c_{N B}\right) D_{N B}^{2}\left(p_{N B}^{2}, p_{P L}^{2}\right)+\left(p_{P L}^{2}-c_{P L, H}\right) D_{P L}^{2}\left(p_{N B}^{2}, p_{P L}^{2}\right)\right] \\
& \text { s.t. : } \quad \Pi\left(p_{N B}^{1}, p_{N B}^{2}, p_{P L}^{1}, p_{P L}^{2}\right) \geq \widetilde{\Pi}\left(\Pi\left(p_{N B}^{1}, p_{P L}^{1}\right) ; \quad D_{P L}^{1}\left(\Pi\left(p_{N B}^{1}, p_{P L}^{1}\right) \geq 0\right. \text {. }\right.
\end{aligned}
$$

Note that the second constraint is different from the one in the profit maximization problem in case $2(\mathrm{~A})$. The one in the present case is used to examine if the retailer 
would sell only the NB product in equilibrium. Again, the profit $\widetilde{\Pi}(\cdot)$ from selling the low-quality product is independent of the second period prices since all the consumers (including the NB customers) will not buy from the retailer in the second period if the retailer has sold the low-quality PL product in the first period. If the PL product is of high quality, on the other hand, consumers in the second period will have perfect information about the quality and make the choice as in the benchmark case. The Lagrange function associated with the above problem can be written as

$\mathscr{L}\left(\mathbf{p}_{N B}, \mathbf{p}_{P L}, \lambda_{1}, \lambda_{2}\right)=\Pi\left(\mathbf{p}_{N B}, \mathbf{p}_{P L}\right)+\lambda_{1}\left[\Pi\left(\mathbf{p}_{N B}, \mathbf{p}_{P L}\right)-\widetilde{\Pi}\left(p_{N B}^{1}, p_{P L}^{1}\right)\right]+\lambda_{2} D_{P L}^{1}\left(p_{N B}^{1}, p_{P L}^{1}\right)$.

The first-order conditions with respect to the retail prices in period 1 are

$$
\begin{aligned}
& \frac{\partial \mathscr{L}(\cdot)}{\partial p_{N B}^{1}}=\left(1+\lambda_{1}\right) \frac{\partial \Pi\left(\mathbf{p}_{N B}, \mathbf{p}_{P L}\right)}{\partial p_{N B}^{1}}-\lambda_{1} \frac{\partial \widetilde{\Pi}\left(p_{N B}^{1}, p_{P L}^{1}\right)}{\partial p_{N B}^{1}}+\lambda_{2} \frac{\partial D_{P L}^{1}\left(p_{N B}^{1}, p_{P L}^{1}\right)}{\partial p_{N B}^{1}}=0, \\
& \frac{\partial \mathscr{L}(\cdot)}{\partial p_{P L}^{1}}=\left(1+\lambda_{1}\right) \frac{\partial \Pi\left(\mathbf{p}_{N B}, \mathbf{p}_{P L}\right)}{\partial p_{P L}^{1}}-\lambda_{1} \frac{\partial \widetilde{\Pi}\left(p_{N B}^{1}, p_{P L}^{1}\right)}{\partial p_{P L}^{1}}+\lambda_{2} \frac{\partial D_{P L}^{1}\left(p_{N B}^{1}, p_{P L}^{1}\right)}{\partial p_{P L}^{1}}=0 .
\end{aligned}
$$

And the first-order conditions with respect to the second period retail prices are the same as those in the benchmark case. By solving simultaneously (1.14) and (1.15) we can obtain

$$
p_{N B}^{1}=p_{N B}^{*} \quad \text { and } \quad p_{P L}^{1}=p_{P L}^{*}+\frac{\lambda_{1}}{2}\left(c_{P L, H}-c_{P L, L}\right)-\frac{\lambda_{2}}{2}
$$

Lemma 1.6 Under imperfect information, if $c_{N B}^{c}<c_{N B}<c_{N B}^{b}$, the retailer always sells a positive amount of the PL products in the first period, i.e., $D_{P L}^{1}\left(p_{N B}^{1}, p_{P L}^{1}\right)>0$.

Lemma 1.6 demonstrates that if the cost of the NB product falls in the intermediate range, the retailer has an incentive to engage in market segmentation despite that 
information is imperfect. Because of the need to signal quality through a higher price, the profitability of selling the PL product in the first period may be smaller under asymmetric information than under perfect information (to be illustrated in Lemma 1.7 below). But doing so is still profitable in its own right. Moreover, it enables the retailer to earn the same level of profit in the second period as that under perfect information (i.e., $\Pi_{N B, P L}^{2 *}$ ). As a result, the retailer's total profit over the two periods from selling both products is greater than that from selling only the NB product.

Lemma 1.7 In the case where $c_{N B}^{c}<c_{N B}<c_{N B}^{b}$, the joint profit associated with the product line $(N B, P L)$ is

$$
\begin{cases}\Pi_{N B, P L}=\hat{\Pi}_{N B, P L} & \text { if } \quad c_{P L, H}^{b} \leq c_{P L, H}<c_{P L, H}^{a} \quad \text { and } \quad c_{N B}^{e} \leq c_{N B}<c_{N B}^{b} \\ \Pi_{N B, P L}=\Pi_{N B, P L}^{*} & \text { if } \quad c_{P L, H}<c_{P L, H}^{b} \quad \text { or } \quad c_{N B}^{c}<c_{N B}<c_{N B}^{e} .\end{cases}
$$

Where $c_{N B}^{e} \in\left(c_{N B}^{c}, c_{N B}^{b}\right)$ and such that

$$
\Lambda\left(c_{N B}^{e}\right)=\delta\left(c_{N B}^{a}-c_{N B}\right)\left(c_{N B}^{b}-c_{N B}\right)+(2+\delta)\left(c_{N B}-c_{N B}^{c}\right)\left(c_{P L, H}^{b}-c_{P L, H}\right)=0,
$$

and the retailer sets price $p_{P L}^{1}=\hat{p}_{P L}\left(\hat{\hat{\lambda}}_{1}\right)$ in the former case. ${ }^{15}$ Note: we use "doublehat" to the profit from $(N B, P L)$ when $c_{P L, H}^{b} \leq c_{P L, H}<c_{P L, H}^{a}$ and $c_{N B}^{e} \leq c_{N B}<c_{N B}^{b}$ to distinguish from the one in case $2(A)$.

Lemma 1.7 imposes more restrictive conditions on the corresponding parameters when the retailer wishes to serve heterogeneous consumers by differentiating its products under imperfect information. Similar to case $2(\mathrm{~A})$, if $c_{P L, H}$ is small enough (i.e., if $\left.c_{P L, H}<c_{P L, H}^{b}\right)$, the retailer will charge both products at the benchmark price level and the equilibrium is same as the one from case 3. If $c_{P L, H}$ exceeds this certain threshold, on the other hand, the retailer would have same incentive as the previous

\footnotetext{
${ }^{15}$ The values of equilibrium prices and profits are provided in the proof of this lemma.
} 
situation if the marginal cost of producing the NB product stays on a lower level (e.g., if $\left.c_{N B}^{c}<c_{N B}<c_{N B}^{e}\right)$. If the cost of the NB product is no smaller than the value $c_{N B}^{e}$, however, the retailer needs to raise the retail price on the PL product to signal the quality, which leads a fall on the equilibrium joint profit.

Due to two scenarios on the marginal cost of the two products, the retailer's bargaining payoff at stage two should also vary on the range of $c_{N B}$ and $c_{P L, H}$. If the joint profit from stage three is on the benchmark level, i.e., $\Pi_{N B, P L}^{*}$, the retailer's bargaining profit will be $\gamma \Pi_{N B, P L}^{*}+(1-\gamma) \Pi_{P L}^{*}$. With the help of Proposition 1.3, this bargaining profit is greater than the one from the other two product lines. On the other hand, if $c_{N B}^{e} \leq c_{N B}<c_{N B}^{b}$ and $c_{P L, H}^{b} \leq c_{P L, H}<c_{P L, H}^{a}$, the retailer's bargaining profit will become to $\gamma \hat{\Pi}_{N B, P L}+(1-\gamma) \hat{\Pi}_{P L}$ and the equilibrium at stage one can be concluded as:

Proposition 1.6 If $c_{N B}^{c}<c_{N B}<c_{N B}^{b}$, the PBE under imperfect information is characterized by the following.

(i) In the case where $c_{P L, H}<c_{P L, H}^{b}$ or $c_{P L, H}^{b} \leq c_{P L, H}<c_{P L, H}^{a}$ and $c_{N B}^{c}<c_{N B}<c_{N B}^{e}$, the product line is $(N B, P L)$ and the retailer sets the prices at $p_{N B}^{1}=p_{N B}^{2}=p_{N B}^{*}$ and $p_{P L}^{1}=p_{P L}^{2}=p_{P L}^{*}$. The belief system is $\{\alpha, \beta\}=\{1,1\}$ if $p_{P L}^{1} \geq p_{P L}^{*}$, and $\{\alpha, \beta\}=$ $\{0,0\}$ if $p_{P L}^{1}<p_{P L}^{*}$. The retailer's equilibrium profit is $\gamma \Pi_{N B, P L}^{*}+(1-\gamma) \Pi_{P L}^{*}-K$.

(ii) In the case where $c_{P L, H}^{b} \leq c_{P L, H}<c_{P L, H}^{a}$ and $c_{N B}^{e}<c_{N B}<c_{N B}^{b}$, the product line is $(N B, P L)$ and the retailer sets the prices at $p_{N B}^{1}=p_{N B}^{2}=p_{N B}^{*}$ and $p_{P L}^{1}=\hat{p}_{P L}\left(\hat{\hat{\lambda}}_{1}\right), p_{P L}^{2}=p_{P L}^{*}$. The belief system is $\{\alpha, \beta\}=\{1,1\}$ if $p_{P L}^{1} \geq \hat{p}_{P L}\left(\hat{\hat{\lambda}}_{1}\right)$, and $\{\alpha, \beta\}=\{0,0\}$ if $p_{P L}^{1}<\hat{p}_{P L}\left(\hat{\hat{\lambda}}_{1}\right)$. The retailer's equilibrium profit is $\hat{\Pi}_{N B, P L}+$ $(1-\gamma) \hat{\Pi}_{P L}-K$.

The equilibrium in Proposition 1.6 is qualitatively the same as Proposition 1.3, which implies that the market segmentation with the PL product is still attractive to 
the retailer even the information is not perfect. Although the bargaining profit from the product line $(\mathrm{NB}, \mathrm{PL})$ to the retailer varies with the marginal costs of the two products, it still dominates the one from the other product lines in equilibrium. In other words, the presence of asymmetric information does not fundamentally change the retailer's incentive to engage in market segmentation.

\subsection{Consumer Welfare}

Since the main interest of this chapter is in brand stretching, our welfare analysis will focus on the situation where the retailer uses the NB product to signal the quality of the PL product, i.e., on case $2(\mathrm{~A})$. Let $C S_{N B, P L}$ and $C S_{P L}$ denote the consumer surplus in the first period from the product line $(\mathrm{NB}, \mathrm{PL})$ and $(0, \mathrm{PL})$, respectively. It can be shown that:

$$
\begin{gathered}
C S_{N B, P L}=\int_{\widetilde{\theta}_{2,0}\left(\hat{\lambda}_{1}\right)}^{\bar{\theta}}\left[s_{N B} \theta-p_{N B}\right] d \theta+\int_{\widetilde{\theta}_{2,0}\left(\hat{\lambda}_{1}\right)}^{\widetilde{\theta}_{1,2}\left(\hat{\lambda}_{1}\right)}\left[s_{P L, H} \theta-p_{P L}\left(\hat{\lambda}_{1}\right)\right] d \theta, \\
C S_{P L}=\int_{\widetilde{\theta}_{2,0}(\hat{\lambda})}^{\bar{\theta}}\left[s_{P L, H} \theta-p_{P L}(\hat{\lambda})\right] d \theta .
\end{gathered}
$$

The impact of brand stretching on consumer welfare can be examined by comparing (1.16) and (1.17). Here we do not need to consider the consumer surplus in the second period because the equilibrium in the second period is the same for both product lines, which, in turn, is the same as that under perfect information.

Proposition 1.7 Under imperfect information, if $c_{N B}^{b} \leq c_{N B}<c_{N B}^{d}$ and $c_{P L, H}^{b} \leq$ $c_{P L, H}<c_{P L, H}^{a}$, then $C S_{N B, P L}>C S_{P L}$.

Proposition 1.7 implies that the consumers can be better-off when the retailer uses the brand stretching strategy. One reason can be seen from Corollary 1.2 in section 1.4, which is, the price distortion on the PL product in the product line (NB, PL) is 
smaller than the one in $(0, \mathrm{PL})$. In other words, a lower retail price on the PL product increases the surplus accrued to those consumers who purchase the PL product.

\subsection{Conclusion}

We have constructed and studied a model of private label product under both perfect information and imperfect information about the quality of the product. In the benchmark case of perfect information, we have shown that the private label product can be used by a retailer to strengthen its bargaining position against the supplier of a national brand product and to segment the market. More specifically, the launch of the private label product raises the retailer's disagreement payoff, which enables the retailer to receive a larger share of their joint profit from the negotiation with the supplier. In the presence of heterogeneous consumers, moreover, the retailer can benefit from enriching its product line with differentiated products that caters to different types of customers.

In an environment of asymmetric information where the quality of the private label product cannot be observed by the consumers before they purchase, we have found that this informational asymmetry does not fundamentally change these two motivations for launching a private label product. Although the profit to the retailer is smaller under some circumstances due to imperfect information, the retailer would still carry the private label product as a way to strengthen its bargaining power and/or engage in market segmentation.

More importantly, we have demonstrated a third motivation for the sale of private label product under asymmetric information, namely, brand stretching. More specifically, the retailer can signal the quality of the private label product by simultaneously selling a positive amount of the national brand product in situations where the cost of the latter is so high that the retailer would not have done so under perfect informa- 
tion. Different from the existing studies on brand stretching (Choi, 1998 and Cabral, 2000) in which both the base and new products are under same brand name, we examine a situation where the informational problem can be alleviated by using the signal from a high-quality product of a different brand. Specifically, the presence of a base product with another brand name (i.e., the national brand product) increases the retailer's punishment for selling a low-quality new product (i.e., the low-quality private label product), which reduces the temptation of selling the inferior private label product. Moreover, the price distortion on the new product with unobservable product quality can be reduced with a proven high-quality base product under a different brand.

Moreover, the conditions in equilibrium we have discussed may change when the assumption on consumers are modified. More specifically, the information about product quality may be partially disseminated among consumers. For instance, if the information about the quality of the private label product can only be conveyed to a fraction of consumers who have not purchased such product, the retailer's decision will depend on the fraction of informed consumers. In such a case, some consumers will still choose to buy from the retailer in period 2 even if the low-quality product was sold in the previous period. Thus the punishment for the retailer's cheating on product quality is reduced. By the continuity of the retailer's incentive compatibility constraint, we may still obtain the same results as the present study when the fraction approaches 1.

The second modification is from the point of view of consumer's reaction to the low-quality product. Specifically, a fraction of national brand customers may continue to purchase from the retailer in the second period although the low-quality private label product is known to all consumers. In such a case, the punishment for the retailer's cheating on the quality of the private label product is also reduced. But, 
similar to the first modification, if the fraction of these consumers is close to zero, we will obtain the same results as the present study.

One question that we will address through an extension of this model is that in the case where the retailer uses the national brand product to signal the quality of the private label product, it needs to sell a positive quantity of the national brand product for only one period. In reality, however, it is rare that a retailer sells the national brand product only in the period when the private label product is initially introduced. Rather, the retailer usually sells both products in a long term once it has decided on a diversified product line. In order to address this issue, we will consider an overlapping-generation model in which consumers live for only two periods and in each period a new cohort of consumers enter the market to replace the old one. In

such a model, the informational asymmetry exists in every period if the quality of the private label product is not known to the new cohort of consumers who have just entered the market. Consequently, the retailer needs the national brand product to signal the quality of the private label product in every period.

\section{Bibliography}

Andersson, F. (2002): "Pooling Reputations," International Journal of Industrial Organization, 20, 715-730.

Avenel, E. AND S. CAPrice (2006): "Upstream Market Power and Product Line Differentiation in Retailing," International Journal of Industrial Organization, 24, $319-334$.

Belleflamme, P. And M. Peitz (2010): Industrial Organization: Markets and Strategies, Cambridge: Cambridge University Press. 
Bergès-Sennou, F. (2006): "Store Loyalty, Bargaining Power and the Private Label Production Issue," European Review of Agricultural Economics, 33, 315-335.

Bergès-Sennou, F., F. Bontems, and V. RÈquillart (2004): "Economics of Private Labels: A Survey of Literature," Journal of Agricultural \& Food Industrial Organization, 2, 1-23.

Bernheim, B. D. And M. D. Whinston (1986): "Common Agency," Econometrica, 54, 923-942.

Bontems, P., S. Monier-Dilhan, And V. Requillart (1999): "Strategic Effects of Private Labels," European Review of Agricultural Economics, 26, 147-165.

Cabral, L. M. B. (2000): "Stretching Firm and Brand Reputation," RAND Journal of Economics, 31, 658-673.

_ (2009): "Umbrella Branding with Imperfect Observability and Moral Hazard," International Journal of Industrial Organization, 27, 206-213.

Cabral, L. M. B. And A. Hortaçsu (2010): "The Dynamics of Seller Reputation: Evidence From EBay," The Journal of Industrial Economics, 58, 54-78.

Champsaur, P. And J. Rochet (1989): "Multiproduct Duopolists," Econometrica, 57, 533-557.

Chevalier, J. A. And D. Mayzlin (2006): "The Effect of Word of Mouth on Sales: Online Book Reviews," Journal of Marketing Research, 43, 345-354.

Chor, J. P. (1998): "Brand Extension as Informational Leverage," Review of Economic Studies, 65, 655-669.

Colangelo, G. (2008): "Private Labeling and Competition between Retailers," Journal of Agricultural and Food Industrial Organization, 6, 1-36. 
Gabrielsen, T. S. and L. Sorgard (2007): "Private Labels, Price Rivalry, and Public Policy," European Economic Review, 51, 403-424.

Gilbert, R. J. And C. Matutes (1993): "Product Line Rivalry with Brand Differentiation," The Journal of Industrial Economics, 41, 223-240.

Harsanyi, J. and R. Selten (1972): "A Generalized Nash Solution for Twoperson Bargaining Games with Incomplete Information," Management Science, 18, 80-106.

Mills, D. E. (1995): "Why Retailers Sell Private Labels," Journal of Economics \&3 Management Strategy, 4, 509-528.

- (1999): "Private Labels and Manufacturer Counterstrategies," European Review of Agriculatural Economics, 26, 125-145.

Mussa, M. And S. Rosen (1978): "Monopoly and Product Quality," Review of Industrial Organization, 18, 301-017.

Narasimhan, C. And R. T. Wilcox (1998): "Private Labels and the Channel Relationship: A Cross-Category Analysis," The Journal of Business, 71, 573-600.

Smith, W. R. (1956): "Product Differentiation and Market Segmentation as Alternative Marketing Strategies," Journal of Marketing, 21, 3-8.

Spengler, J. J. (1950): "Vertical Integration and Antitrust Policy," Journal of Political Economy, 58, 347-352.

Tadelis, S. (1999): "Whats in a Name? Reputation as a Tradable Asset," The American Economic Review, 89, 548-563.

Tirole, J. (1988): The Theory of Industrial Organization, The MIT Press. 
Weinstein, A. (2004): Handbook of Market Segmentation: Strategic Targeting for Business and Technology Firms, Routledge. 


\section{Chapter 2}

\section{Private Labels and Product}

\section{Quality under Asymmetric}

\section{Information: An}

\section{Overlapping-Generation Model}

\section{$2.1 \quad$ Introduction}

In a two-period model of asymmetric information about the product quality, we have studied the motivations of a retailer to launch a private label product. Particularly, the launch of the private label product can be viewed as a form of brand stretching, namely, with the sales of the national brand product acting as a bond to ensure that the retailer sells only the high-quality private label product. Consequently, the retailer would carry and sell the national brand product even in the situation where it would have not done so under perfect information due to a relatively high production 
cost. $^{1}$

There is one issue, however, that cannot be addressed in the two-period model. That is, in the case where the retailer uses brand stretching, it carries and sells the national brand product to signal the quality of the private label product for only one period (i.e., the first period only). In reality, it is rare that a retailer sells the national brand product only in the period when its own branded product is initially introduced. Rather, the retailer usually offers both types of products on a long-term basis. Indeed, a report from Information Resources Inc. indicates that the national brand unit share of consumer packaged goods in the U.S. remained almost unchanged during the 2010-2013 period, which was around 82.7 percent. This implies that in the long term, the sales of national brand products are quite stable. In terms of beverage category (in the U.S.), for example, the national brand unit share in 2014 increased by 0.1 percent (in grocery channel) with respect to the previous year, and the data in 2013 was the same.

One explanation for this phenomenon is consumer loyalty to the national brand. In order to retain those loyal consumers, the retailer has to offer the national brand product all the time. This explanation has been studied in Narasimhan and Wilcox (1998), Bergès-Sennou (2006), and Gabrielsen and Sorgard (2007). These studies, however, are conducted under the assumption of perfect information about the product quality. Their main focus is on how the retailer can benefit from market segmentation. In a world of imperfect information, on the other hand, the sales of the national brand product may be explained from another angle, i.e., signaling the private label product is of high quality in a long term.

Therefore, the objective of this chapter is to investigate the incentives of a retailer

\footnotetext{
${ }^{1}$ As shown in the two-period model, a retailer may have two other motivations to launch the private label product, i.e., to strengthen its bargaining power against the national brand supplier and to segment the market. In this chapter, we are particularly interested in the retailer's motivation of using the brand stretching strategy.
} 
to use a national brand product to signal the quality of a private label product in the long term when the quality of the latter is not observed by the consumers prior to consumption. By constructing an overlapping-generation (OLG) model, we derive the conditions under which the retailer uses the national brand product to signal the quality of the private label product in the long term.

To be more specific, we consider a model in which each cohort of consumers is for two periods. They enter the market in the first period of their lives and make purchase in each period of life. If some consumers purchase the private label product in the first period of their lives, the actual quality of the private label product becomes known in the next period to all consumers in this cohort. Thus, these consumers make their second purchase based on their updated belief about the product quality. The consumers leave the market after their second period of life.

Furthermore, a cohort of new consumers enters the market in every period but they are unable to communicate with the previous cohort. ${ }^{2}$ As a consequence, the new consumers do not know the actual quality of the private label product even though their old counterparts have already updated their belief about the product quality. This implies that the problem of asymmetric information exists in every period since the information dissemination is confined to the consumers in the same cohort only.

In terms of the product line, the retailer can offer both national brand and private label product, or offer only one of the two products. The quality of the private label product is determined by the retailer in the period when such product is initially introduced and it can be either high or low. The retailer would not sell a low-quality private label product in a world of perfect information, but it may have incentives to do so in the case where consumers cannot observe the quality before purchase. We

\footnotetext{
${ }^{2}$ Note that the new cohort of consumers is not necessarily the offspring of the old one. Accordingly, communication may not take place between the two cohorts of consumers. One example of such a situation would be newcomers (e.g., immigrants) to a new city who may not be familiar with the private label products offered by the local retailers and they do not have any friends in the city.
} 
will examine the case where the retailer carries and sells both national brand and (high-quality) private label products in the presence of this moral hazard problem. We show that, in the case where the cost of the national brand product is high relative to that of the private label product, the retailer does not have incentive to carry the former under perfect information but may do so in a world of imperfect information. This is because (under some certain conditions) the presence of the national brand product can be a credible signal for the quality of the private label product (i.e., brand stretching). Furthermore, the retailer may use this brand stretching strategy in every period once it has done so in the first period.

Figure 2.1 illustrates the specific equilibriums for different ranges of parameter values. It shows that the retailer's equilibrium product line depends crucially on the relative production costs of the national brand and (high-quality) private label products (denoted by $c_{N B}$ and $c_{P L, H}$ respectively). Specifically, if the parameter values fall into region $I$, i.e., if $c_{P L, H}<c_{P L, H}^{b}$, the retailer will sell only the private label products. On the other hand, if the cost of the private label product is very high, i.e., if $c_{P L, H}^{c} \leq c_{P L, H}<c_{P L, H}^{a}$, the retailer will sell only the national brand products in equilibrium (i.e., in region $V$ ). If the cost of the private label product is intermediate, i.e., if $c_{P L, H}^{b} \leq c_{P L, H}<c_{P L, H}^{c}$, the retailer will sell both national brand and private label product in every period when the cost of the former is below a threshold $c_{N B}^{c}$ (i.e., in region $I I)$. If $c$ exceeds that threshold but below $c_{N B}^{d}$, i.e., in region $I I I$, the retailer will sell both products only in period 1 and sell only the private label product in the following periods. If $c_{N B}$ is above $c_{N B}^{d}$ (in region $I V$ ), selling both products is not profitable to the retailer. Accordingly, it will sell only the private label product but earns a reduced profit relative to that in region $I$ since the cost of the private label product in this case is greater than $c_{P L, H}^{b}$.

The rest of this chapter is organized as follows. Section 2.2 introduces the model. 
Figure 2.1: The retailer's equilibrium product line under imperfect information

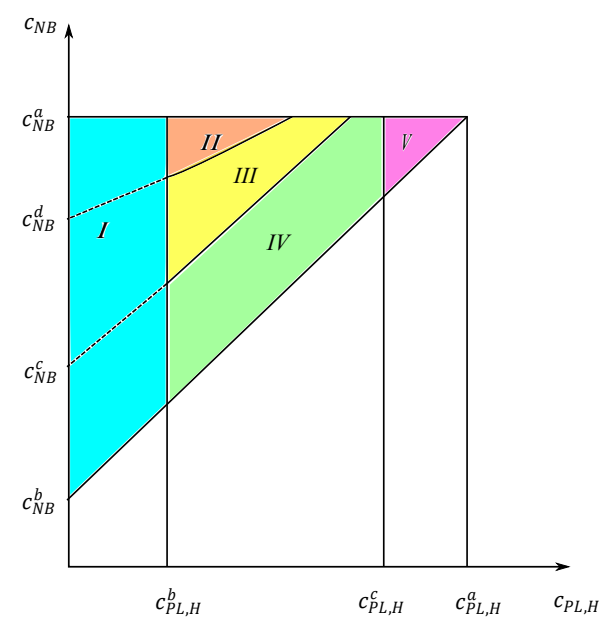

Section 2.3 briefly discusses a benchmark case of perfect information. Section 2.4 solves the model under imperfect information and presents the mains results. Section 2.5 concludes.

\section{$2.2 \quad$ The Model}

\subsubsection{The Firms}

The basic setup of the model is the same as the one in the two-period model. There are two levels of markets. In the upstream market, a manufacturer (firm 1) produces a national brand (NB hereafter) consumer product with quality $s_{N B}$ at a constant marginal cost $c_{N B}$. In the downstream market, a retailer (firm 2) buys and resells the NB products at a retail price $p_{N B}$. The retailer, meanwhile, has the option of producing and selling a private label (PL hereafter) product at price $p_{P L}$. The PL product is an imperfect substitute for the NB product in that it has a lower quality. To be more specific, we assume there are two possible levels of quality for the PL 
product, high $\left(s_{P L, H}\right)$ and low $\left(s_{P L, L}\right)$, with $s_{N B}>s_{P L, H}>s_{P L, L}$. The retailer can produce a high-quality PL product at marginal $\operatorname{cost} c_{P L, H}$ or a low-quality PL product at marginal cost $c_{P L, L}$, with $c_{N B}>c_{P L, H}>c_{P L, L}$.

Suppose there are no barriers to entry to the production and sales of the low quality product. Accordingly, there are a large number of firms that produce and sell a perfect substitute for the low-quality PL product. In the case where the quality of the PL product is known to consumers, the low-quality PL product can only be sold at a price equaling $c_{P L, L}$ due to the competition from the other producers. For ease of discussion, we will refer to this low-quality substitute as the no-name (NN hereafter) product (this vertical relationship among the firms is described in Figure 1.3 in Chapter 1 of this thesis).

In the event that the retailer chooses to launch the PL product (regardless of high or low quality), it has to incur a sunk cost $K$ to design and develop the product. We assume that $K$ is small but positive. This assumption ensures that, in the case where consumers have perfect information about quality, the retailer does not have any incentive to introduce the low-quality private label product since it would not be able to recover the cost $K$.

Suppose that the contract between the supplier and the retailer takes the form of two-part tariff $T=(w, F)$, where $w$ denotes the wholesale price on the NB product and $F$ denotes a fixed fee charged to the retailer. The terms of the contract are determined by the Generalized Nash Bargaining solution (details to be discussed later).

In term of observability on the product quality, we assume that the quality of the NB and the NN products is public information while that of the PL product can be ascertained only after it is consumed by at least some consumers. The NB supplier, however, is able to find out the quality of the PL product through the negotiation 
process. This is because in the event that the retailer wants to use the PL product as the threat point in the negotiation process, it will have to present credible evidence to show that it has developed the PL product, and as a professional manufacturer the NB supplier will be able to ascertain the quality of the product from the evidence presented by the retailer.

\subsubsection{The Consumers}

Consider an overlapping generation model in which a new generation of consumers enters the market in every period and each consumer lives for two periods. Time is denoted by $t=1,2, \ldots$ In period 1 , all consumers are new to this market. Both new and old consumers co-exist in all subsequent periods. The flow of consumers is

illustrated in Figure 2.2, where $G_{t}^{l}(t \geq 1$ and $l=1,2)$ represents the consumers who

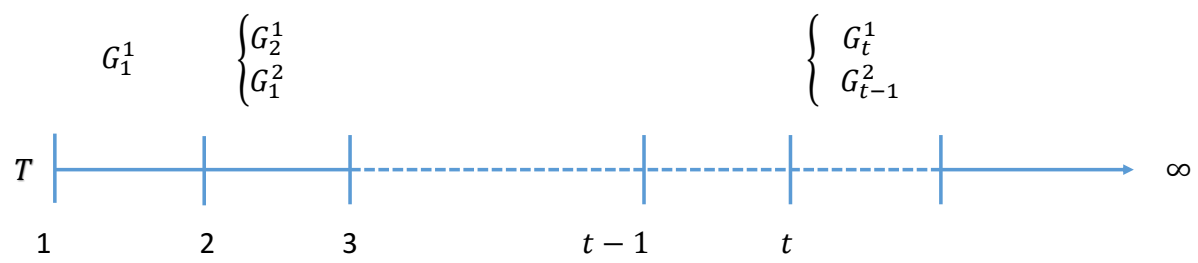

The old consumers know the true quality of the PL product as long as some of them have consumed this product in their first period of life. The new consumers, however, do not know the quality of the PL product. We assume that there is no communication between the two generations in any period. As a consequence, the information about quality can only be shared within the generation but not between 
the generations.

Following the quality-choice model of Mussa and Rosen (1978), we assume that consumers in each generation are indexed by their preferences which are uniformly distributed on a vertical interval $[0, \bar{\theta}]$. The distribution has constant density $1 / \bar{\theta}$ over the interval so that the measure is 1 . Consumers at different locations on the interval have different tastes for quality. Specifically, a consumer $k$ (regardless which generation she comes from) has a net surplus (indirect utility)

$$
u^{k}=s_{i} \theta^{k}-p_{i}, \quad \theta^{k} \in[0, \bar{\theta}]
$$

if she purchases a product of quality $s_{i}$ at price $p_{i}$, and $u^{k}=0$ if she does not purchase anything. The population size of each generation is constant over time and is normalized to 1 . This implies that the total population is 1 in the first period, and 2 in all subsequent periods. The demand for a product by consumers of each generation can be derived by determining the location of the marginal consumer who is indifferent between buying this good and the adjacent one. Therefore, the locations of marginal consumers in a two-good (suppose good $i$ and $j$ with good $i$ being of higher quality) market can be determined by equating the net surplus from purchasing two adjacent products:

$$
\tilde{\theta}_{i j}=\frac{p_{i}-p_{j}}{s_{i}-s_{j}} \quad \text { and } \quad \tilde{\theta}_{j 0}=\frac{p_{j}}{s_{j}}
$$

where $\widetilde{\theta}_{i j}$ denotes the location of marginal consumers who are indifferent between buying good $i$ and $j ; \widetilde{\theta}_{j 0}$ denotes the location of marginal consumers who are indifferent between buying good $j$ and nothing. Then the demand for each product by a generation is determined by the locations of those marginal consumers as follows:

$$
D_{i}\left(p_{i}, p_{j}\right)=\max \left\{0, \bar{\theta}-\widetilde{\theta}_{i j}\right\} ; \quad D_{j}\left(p_{i}, p_{j}\right)=\max \left\{0, \widetilde{\theta}_{i j}-\widetilde{\theta}_{j 0}\right\}
$$


The demand functions in (2.2) imply that the quantity of a product would be zero if its retail price is above a certain level. ${ }^{3}$ In addition, because the lower bound of $\theta$ is 0 , there will always be a group of consumers with very low values of $\theta$ who will not purchase even the low quality good at any positive price. In other words, the market will not be fully served in equilibrium.

\subsubsection{The Timing}

While consumers in each generation live only two periods, firms are in the market forever. The time discount factors of all consumers and firms are the same, denoted by $\delta \in(0,1)$. In period 1 , the retailer chooses the product line and, if the latter includes the national brand product, negotiates a contract with the NB manufacturer that covers period 1 as well as all subsequent periods. While the retailer is permitted to add or delete a product from its product line in any period after period 1, such future addition or deletion will be anticipated and thus taken into consideration in the contract between the retailer and the NB manufacturer. In addition, we assume that the NB supplier does not take strategic actions to counter the retailer's use of PL to gain bargaining power.

To be more specific, the game in period 1 has three stages. At stage one, the retailer makes a decision on its product line. Because there are two products (i.e., $\mathrm{NB}$ and $\mathrm{PL}$ ) that can be sold through the retailer, the retailer chooses from the following three possible product lines, indexed by (NB, 0), (0, PL) and (NB, PL). The first two product lines contain only one product, NB only or PL only, while the last product line consists of both products. The retailer incurs the cost $K$ if it chooses a product line that includes the PL product. The quality of the PL product is also determined at this stage and, the quality cannot be changed in subsequent periods.

\footnotetext{
${ }^{3}$ Note that $\partial D_{i} / \partial p_{i}=\left(\partial D_{i} / \partial \widetilde{\theta}_{i j}\right) \cdot\left(\partial \widetilde{\theta}_{i j} / \partial p_{i}\right)<0$. Similarly, $\partial D_{j} / \partial p_{j}<0$.
} 
At stage two, if the retailer has chosen to carry the NB product as part of its product line, it negotiates a contract with the NB supplier. Moreover, we assume that the firms have rational expectations such that they can correctly anticipate their future profits in period 1 and beyond. If the two firms fail to reach an agreement, the retailer would sell only the PL product in period 1 as long as it has launched such product at stage one. Otherwise, the retailer will sell nothing in the current period but launch and sell the PL product (only) from period 2 onwards. In either case, the retailer will obtain a disagreement payoff that depends on the cost of the PL product. Moreover, the disagreement payoff to the NB supplier is zero.

At stage three, the retailer sets the retail prices of the product(s) for period 1 , and consumers make their purchase decisions.

In each subsequent period (i.e., for $t \geq 2$ ), the retailer can change its product line by adding a or dropping a product. For example, if the NB product is offered in the previous period, the retailer could choose to remove it in the current period. Alternatively, if the retailer did not offer a PL product in the previous period, it could add it in the current period by incurring the cost $K$. After the decision on the product line, the retailer sets the retail prices for this period.

On the consumer side, the old generation can use their consumption experience in the previous period to update their belief about the product quality provided that the retailer has kept the same product line. Otherwise, they will not be able to update their beliefs. Meanwhile, a new generation enters the market and makes their purchase decision based on their belief about the product quality.

We assume that if the retailer sells the low-quality PL product at an inflated price (i.e., at a price greater than $c_{P L, L}$ ) in a period, it will lose all the old customers in the following period, including those who purchased the NB product previously. But the new customers in the later period may still purchase from the retailer since 
information cannot be shared between the generations.

The game between the firms and the (new) consumers in period 1 has been illustrated in Figure 1.4 in Chapter 1 of this thesis. Note that the consumers do not know the quality of the PL product when they observe the product line (NB, PL) and (0, PL) in the first period of their lives. Parameters $\alpha \in[0,1]$ and $\beta \in[0,1]$ denote the consumers' (in their first period of life) belief about the quality of the PL product when one of these two product lines is offered. Specifically, $\alpha$ and $\beta$ respectively represent the probabilities that the PL product in the product line (NB, PL) and (0, PL) is of high quality. Moreover, when either of these two product lines is available, a consumer's choice will be "buy" or "not buy" from this retailer depending on her taste. As we have assumed, the consumers who do not buy from this retailer will buy the NN product or nothing. In the case where the retailer offers the product line (NB, PL), the consumer also makes a decision on which product she wants to buy. Otherwise, the consumer has only one option when she purchases from the retailer.

Since the retailer faces a stationary environment after period 1, we will focus on a stationary strategy with which the retailer chooses the same strategy in all periods starting from $t \geq 2$. This implies that the retailer will offer the same product line and set the same retail prices in all these periods. Thus, any changes in the product line or the retail prices will take place only between period 1 and period 2 .

\subsubsection{The Contract Between Firms}

Suppose that the terms of contract $T_{t}=\left(w_{t}, F_{t}\right)$ between the retailer and the NB supplier are determined by the Generalized Nash Bargaining solution (Harsanyi and Selten, 1972), where $w_{t}$ and $F_{t}(t=1,2, \ldots)$ represent the wholesale price on the NB product and a lump sum payment made in period $t$, respectively. Because the negotiation between the two firms only takes place in period 1 and the firms have 
rational expectation, it determines the terms of contracts in every period all at once.

To be more specific, let $\Pi$ denote the discounted sum of joint profits between the retailer and the NB supplier, and $\pi_{i}^{0}$ denotes firm $i$ 's discounted sum of payoffs in the event of a disagreement. At stage two in period 1, the fixed fee $F$ that the retailer pays to the supplier can be solved from the following Generalized Nash Bargaining problem:

$$
F^{*}=\arg \max _{\pi_{1}}\left(\pi_{1}-\pi_{1}^{0}\right)^{1-\gamma}\left(\pi_{2}-\pi_{2}^{0}\right)^{\gamma} \quad \text { s.t. : } \pi_{1}+\pi_{2}=\Pi,
$$

where $\gamma \in(0,1)$ is a parameter that measures the retailer's bargaining power relative to that of the national brand supplier. This yields the familiar solution:

$$
\pi_{1}^{*}=(1-\gamma) \Pi+\gamma \pi_{1}^{0}-(1-\gamma) \pi_{2}^{0} \quad \text { and } \quad \pi_{2}^{*}=\gamma \Pi-\gamma \pi_{1}^{0}+(1-\gamma) \pi_{2}^{0}
$$

In the special case where both disagreement payoffs are zero, the bargaining power $(\gamma)$ alone determines each firm's share of the joint profit.

With the use of two-part tariff, the contract between the retailer and the supplier is efficient. To be more specific, they will agree on setting wholesale prices $w_{t}$ that maximizes their joint profit. Given the presence of double-marginalization problem (Spengler, 1950), the wholesale prices are set to the marginal cost of the national brand product, i.e., $w_{t}=c_{N B} \cdot{ }^{4}$

\footnotetext{
${ }^{4}$ For more details about the double-marginalization problem and nonlinear pricing, see Tirole (1988), chapter 4; Belleflamme and Peitz (2010), chapter 17.
} 


\subsection{The Equilibrium under Perfect Information: A Benchmark}

In this section, we study the equilibrium in an environment where the product quality can be fully observed by all the agents in the market. That is, all consumers know the true quality of the PL product in both periods of their lives. As we discussed in section 2.1, under perfect information, the retailer does not have any incentive to sell the low-quality PL product since it will obtain a strictly negative profit due to the presence of the developing cost $K$. Thus, we can exclude the low-quality PL product from the analysis in this section.

In the following sub-sections, we will discuss the equilibriums at different stages of the game. Using backward induction, we start with an analysis of the equilibrium starting from period 2. We can do so because the market environment is stationary from period 2 onwards and under perfect information, there is no need for consumers to update their beliefs about the quality of the PL product.

\subsubsection{The Equilibrium From Period 2 Onwards}

This sub-section is to show that the product line will not change from period 2 onwards. Let $D_{N B}\left(p_{N B}^{t}, p_{P L}^{t}\right)$ and $D_{P L}\left(p_{N B}^{t}, p_{P L}^{t}\right)$ be the demand function for the NB and $\mathrm{PL}$ product in period $t \geq 2$, respectively. The retailer's profit maximization problem from period 2 onwards can be expressed by

$$
\max \Pi\left(p_{N B}^{t}, p_{P L}^{t}\right)=\sum_{t=2}^{\infty} 2 \delta^{(t-1)}\left[\left(p_{N B}^{t}-c_{N B}\right) D_{N B}\left(p_{N B}^{t}, p_{P L}^{t}\right)+\left(p_{P L}^{t}-c_{P L, H}\right) D_{P L}\left(p_{N B}^{t}, p_{P L}^{t}\right)\right]
$$


Solving the above problem yields that for any $t$,

$$
D_{N B}\left(p_{N B}^{t}, p_{P L}^{t}\right)+\left(p_{N B}^{t}-c_{N B}\right) \frac{\partial D_{N B}\left(p_{N B}^{t}, p_{P L}^{t}\right)}{\partial p_{N B}^{t}}+\left(p_{P L}^{t}-c_{P L, H}\right) \frac{\partial D_{P L}\left(p_{N B}^{t}, p_{P L}^{t}\right)}{\partial p_{N B}^{t}}=0
$$

and

$$
D_{P L}\left(p_{N B}^{t}, p_{P L}^{t}\right)+\left(p_{P L}^{t}-c_{P L, H}\right) \frac{\partial D_{P L}\left(p_{N B}^{t}, p_{P L}^{t}\right)}{\partial p_{P L}^{t}}+\left(p_{N B}^{t}-c_{N B}\right) \frac{\partial D_{N B}\left(p_{N B}^{t}, p_{P L}^{t}\right)}{\partial p_{P L}^{t}}=0 .
$$

The above two first-order-conditions show that the equilibrium prices are not changed from period 2 onwards. Given that the demand functions are identical through periods, the product line remains the same from period 2 onwards.

\subsubsection{The Product Line in Period 2}

This sub-section is to discuss the retailer's decision on product line in period 2 for a given product line chosen in period 1. More specifically, we examine the possibility that the retailer changes its product line by dropping or adding a product in period 2. With the help of (2.2), we can derive the demand functions for the NB and PL product in period $t \geq 1$ respectively

$$
\left\{\begin{array}{l}
D_{N B}\left(p_{N B}^{t}, p_{P L}^{t}\right)=\max \left\{0, \bar{\theta}-\frac{p_{N B}^{t}-p_{P L}^{t}}{s_{N B}-s_{P L, H}}\right\}, \text { if the PL is present; } \\
D_{N B}\left(p_{N B}^{t}\right)=\max \left\{0, \bar{\theta}-\frac{p_{N B}^{t}-c_{P L, L}}{s_{N B}-s_{P L, L}}\right\}, \text { if the PL is absent }
\end{array}\right.
$$

and

$$
\left\{\begin{array}{l}
D_{P L}\left(p_{N B}^{t}, p_{P L}^{t}\right)=\max \left\{0, \frac{p_{N B}^{t}-p_{P L}^{t}}{s_{N B}-s_{P L, H}}-\frac{p_{P L}^{t}-c_{P L, L}}{s_{P L, H}-s_{P L, L}}\right\}, \text { if the NB is present; } \\
D_{P L}\left(p_{P L}^{t}\right)=\max \left\{0, \bar{\theta}-\frac{p_{P L}^{t}-c_{P L, L}}{s_{P L, H}-s_{P L, L}}\right\} \text { if the NB is absent. }
\end{array}\right.
$$


Since the Nash bargaining solution is efficient, the retailer maximizes the joint profit when choosing the prices for all periods. Recalling that the retailer serves only one generation in period 1 , we write the profit maximization problem as

$$
\begin{array}{r}
\max \Pi\left(p_{N B}^{1}, p_{P L}^{1}, p_{N B}^{t}, p_{P L}^{t}\right)=\left(p_{N B}^{1}-c_{N B}\right) D_{N B}\left(p_{N B}^{1}-p_{P L}^{1}\right)+\left(p_{P L}^{1}-c_{P L, H}\right) D_{P L}\left(p_{N B}^{1}, p_{P L}^{1}\right) \\
+\sum_{t=2}^{\infty} 2 \delta^{(t-1)}\left[\left(p_{N B}^{t}-c_{N B}\right) D_{N B}\left(p_{N B}^{t}, p_{P L}^{t}\right)+\left(p_{P L}^{t}-c_{P L, H}\right) D_{P L}\left(p_{N B}^{t}, p_{P L}^{t}\right)\right]
\end{array}
$$

$$
\text { s.t. : } D_{N B}\left(p_{N B}^{1}, p_{P L}^{1}\right) \geq 0 ; D_{P L}\left(p_{N B}^{1}, p_{P L}^{1}\right) \geq 0 ; D_{N B}\left(p_{P L}^{t}, p_{N B}^{t}\right) \geq 0 ; D_{P L}\left(p_{N B}^{t}, p_{P L}^{t}\right) \geq 0 \text {. }
$$

Note that (2.5) covers all possibilities of the change on the product line in period 2. More specifically, if a product is added (dropped) in a certain period, the corresponding demand will be positive (zero) in that period. Accordingly, we can examine such case by letting the corresponding constraint in (2.5) be slack (binding). Mathematically, this approach is equivalent to that directly lets the demand for a product be positive or zero in the objective function of the problem (2.5), i.e., using the alternative demand function from (2.3) and (2.4) in an unconstrained maximization problem.

Note that with the stationary market environment from period 2 onwards, we are allowed to aggregate the retailer's profits after period 1 (see section 2.3.1). The Lagrangian function associated with problem (2.5) is

$$
\begin{aligned}
\mathscr{L}=\Pi\left(p_{N B}^{1}, p_{P L}^{1}, p_{N B}^{t}, p_{P L}^{t}\right) & +\mu_{1}^{1} D_{N B}\left(p_{N B} 1, p_{P L} 1\right)+\mu_{2}^{1} D_{P L}\left(p_{N B}^{1}, p_{P L}^{1}\right) \\
& +\mu_{1}^{t} D_{N B}\left(p_{N B}^{t}, p_{P L}^{t}\right)+\mu_{2}^{t} D_{P L}\left(p_{N B}^{t}, p_{P L}^{t}\right)
\end{aligned}
$$

where $\mu_{1}^{1}, \mu_{2}^{1}, \mu_{1}^{t}$ and $\mu_{2}^{t}$ are non-negative Lagrangian multipliers. Solving (2.6) 
yields the equilibrium prices

$$
\left\{\begin{array} { l } 
{ p _ { N B } ^ { 1 } = p _ { N B } ^ { * } - \frac { \mu _ { 1 } ^ { 1 } } { 2 } } \\
{ p _ { P L } ^ { 1 } = p _ { P L } ^ { * } - \frac { \mu _ { 2 } ^ { 1 } } { 2 } }
\end{array} \text { and } \left\{\begin{array}{l}
p_{N B}^{t}=p_{N B}^{*}-\frac{\mu_{1}^{t}}{2} \\
p_{P L}^{t}=p_{P L}^{*}-\frac{\mu_{2}^{t}}{2}
\end{array}\right.\right.
$$

where $p_{N B}^{*}=\bar{\theta}\left(s_{N B}-s_{P L, L}\right) / 2+c_{N B} / 2+c_{P L, L} / 2$ and $p_{P L}^{*}=\bar{\theta}\left(s_{P L, H}-s_{P L, L}\right) / 2+c_{P L, H} / 2+$ $c_{P L, L} / 2$.

Lemma 2.1 Under perfect information, the retailer does not change the product line in period 2 once it has reached an agreement with the $N B$ supplier in period $1 .^{5}$

Lemma 2.1 implies that under perfect information, the retailer behaves consistently on its product line with unchanged marginal costs and demand through periods. Moreover, Lemma 2.1 excludes the situation that the retailer fails to reach an agreement with the NB supplier in period 1 . In such a case, the retailer may change its product line in period 2 since it may no longer carry and sell the NB product anymore.

\subsubsection{The Equilibrium at Stage Three in Period 1}

Since the retailer chooses from three possible product lines, we discuss the equilibrium with three different scenarios. Specifically, the retailer maximizes the joint profit at this stage.

\footnotetext{
${ }^{5}$ The proofs of all lemmas and propositions are in appendix.
} 


\subsubsection{The Product Line $(\mathrm{NB}, 0)$}

The retailer sells only the NB product in this sub-game and with the help of (2.3), we can derive the profit maximization problem is:

$$
\max \Pi\left(p_{N B}^{1}, p_{N B}^{t}\right)=\left(p_{N B}^{1}-c_{N B}\right) D_{N B}\left(p_{N B}^{1}\right)+\sum_{t=2}^{\infty} 2 \delta^{(t-1)}\left(p_{N B}^{t}-c_{N B}\right) D_{N B}\left(p_{N B}^{t}\right)
$$

Solving the problems yields the equilibrium retail prices

$$
p_{N B}^{1}=p_{N B}^{t}=p_{N B}^{*}
$$

Note that in equilibrium, the retail prices (on the NB product) are same in all periods although the demand in period 1 is half of that in all subsequent periods.

Substituting the equilibrium price into the demand functions in (2.8), we can derive the equilibrium demand for the NB product (in any generation)as $D_{N B}^{*}=$ $\bar{\theta}-\left(c_{N B}-c_{P L, L}\right) /\left(s_{N B}-s_{P L, L}\right)$. In order to ensure positive demand for the NB product in the product line $(\mathrm{NB}, 0)$, we assume the following

Assumption 2.1 The upper-bound on the marginal cost of the $N B$ product is $c_{N B}^{a}=$ $\bar{\theta}\left(s_{N B}-s_{P L, L}\right)+c_{P L, L}$.

Given Assumption 2.1, we derive the equilibrium joint profit from the product line $(\mathrm{NB}, 0)$ at stage three as:

$$
\Pi_{N B}^{*}=\frac{1+\delta}{1-\delta} \frac{1}{s_{N B}-s_{P L, L}}\left(c_{N B}^{a}-c_{N B}\right)^{2} .
$$

One can easily verify that this equilibrium joint profit is convex in $c_{N B}$, and reaches the lowest level (i.e., $\Pi_{N B}^{*}=0$ ) when $c_{N B}$ equals to $c_{N B}^{a}$. Therefore, this equilibrium profit is decreasing in $c_{N B}$ as long as the value of $c_{N B}$ satisfies Assumption 2.1. 


\subsubsection{The Product Line $(0, \mathrm{PL})$}

In the sub-game where the retailer sells only the PL product, using (2.4), we derive profit maximization problem is

$$
\max \Pi\left(p_{P L}^{t}\right)=\left(p_{P L}^{1}-c_{P L, L}\right) D_{P L}\left(p_{P L}^{1}\right)+\sum_{t=2}^{\infty} 2 \delta^{(t-1)}\left(p_{P L}^{t}-c_{P L, H}\right) D_{P L}\left(p_{P L}^{t}\right)
$$

Solving the problems yields

$$
p_{P L}^{1}=p_{P L}^{t}=p_{P L}^{*}
$$

Substituting the equilibrium price of the PL product into the demand function in (2.9), we obtain the equilibrium demand for the PL product (in any generation) is:

$D_{P L}^{*}=\bar{\theta}-\left(c_{P L, H}-c_{P L, L}\right) /\left(s_{P L, H}-s_{P L, L}\right)$. In order to ensure positive equilibrium demand for the PL product in the product line $(0, \mathrm{PL})$, we impose the following assumption

Assumption 2.2 The upper-bound on the marginal cost of the PL product is $c_{P L, H}^{a}=$ $\bar{\theta}\left(s_{P L, H}-s_{P L, L}\right)+c_{P L, L}$.

This assumption guarantees that selling the (high-quality) PL product can be profitable to the retailer under perfect information. It can be verified that $c_{N B}^{a}-c_{P L, H}^{a}=$ $\bar{\theta}\left(s_{N B}-s_{P L, H}\right)>0$. Thus, Assumption 2.1 and 2.2 can both be satisfied despite the restriction on the relative magnitudes of $c_{N B}$ and $c_{P L, H}$ (namely, $c_{N B}>c_{P L, H}$ ).

Given Assumption 2.2, the retailer's equilibrium profit at stage three is:

$$
\Pi_{P L}^{*}=\frac{1+\delta}{1-\delta} \frac{1}{s_{P L, H}-s_{P L, L}}\left(c_{P L, H}^{a}-c_{P L, H}\right)^{2}
$$

Furthermore, the product line $(0, \mathrm{PL})$ could be chosen in period 2 in the event that the retailer fails to reach an agreement with the NB supplier on the product line $(\mathrm{NB}, 0)$ in period 1 . In such a situation, the price in each period will be the same 
as $p_{P L}^{*}$. The retailer's profit, however, may be reduced depending on its product line in period 1. In this case, the retailer does not sell anything in period 1, i.e., it earns zero profit in period 1. The retailer's profit can therefore be derived as

$$
\Pi_{P L}^{-1 *}=\frac{2 \delta}{1-\delta} \frac{1}{s_{P L, H}-s_{P L, L}}\left(c_{P L, H}^{a}-c_{P L, H}\right)^{2} .
$$

\subsubsection{The Product Line (NB, PL)}

In this sub-game, the retailer carries both products. The profit maximization problem and the equilibrium prices have been provided in (2.5)-(2.7).

In this chapter, we are particularly interested in the situation that the retailer does not carry the NB product under perfect information, but it does so under imperfect information. Accordingly, we let the first and the third constraint in (2.5) be binding in equilibrium. That is, $D_{N B}\left(p_{N B}^{1}, p_{P L}^{1}\right)=D_{N B}\left(p_{N B}^{t}, p_{P L}^{t}\right)=0$ and, hence, $\mu_{1}^{1} \geq 0$ and $\mu_{1}^{t} \geq 0$. If the retailer can still sell the PL product, then the second and the last constraint in (2.5) has to be slack, namely, $\mu_{2}^{1}=\mu_{2}^{t}=0$. The equilibrium prices in (2.7) become: $p_{N B}^{1}=p_{N B}^{t}=p_{N B}^{*}-\mu_{1} / 2, \mu_{1}^{1}=\mu_{1}^{t}=\mu_{1}$ and $p_{P L}^{1}=p_{P L}^{t}=p_{P L}^{*}$.

Lemma 2.2 Under perfect information, the equilibrium demand for the NB product is zero in the product line $(N B, P L)$ if the marginal cost of the NB product satisfies $c_{N B}^{b} \leq c_{N B}<c_{N B}^{a}$.

Lemma 2.2 defines a range of $c$ such that only the equilibrium quantity of the PL product is positive in the product line (NB, PL). That implies, under perfect information, the retailer does not wish to carry the NB product if such cost is very high relative to that of the PL product. Furthermore, when the conditions in Lemma 2.2 hold, one can find that the joint profit from the product line (NB, PL) at stage three in period 1 is identical to that from $(0, \mathrm{PL})$, i.e., $\Pi_{N B, P L}=\Pi_{P L}^{*}$. In order to investigate the retailer's motivation for launching the PL product under imperfect 
information, we assume that the conditions presented in Lemma 2.2 always hold in the subsequent analysis. Specifically, we expect that the retailer may wish to carry and sell the NB product in the long-term under imperfect information even it would have not done so under perfect information. Thus the conditions in Lemma 2.2 help to compare the situations with respect to the information asymmetry.

\subsubsection{The Equilibrium at Stage Two in Period 1}

Recall that the negotiation between the retailer and the supplier takes place only if the retailer chooses a product line that contains the NB product. There are two such product lines, $(\mathrm{NB}, 0)$ and $(\mathrm{NB}, \mathrm{PL})$. Below we discuss each of these two cases in sequence.

If the retailer has chosen the product line $(\mathrm{NB}, 0)$ at stage 1 , it will not be able to sell anything in period 1 in the event of a disagreement with the NB supplier. Instead, it has to wait till period 2 to launch and sell a PL product. Hence, the retailer's disagreement payoff in this case is $\Pi_{P L}^{-1 *}-\delta K$, where $\Pi_{P L}^{-1 *}$ has been introduced in 2.3.3.2. Then the generalized Nash bargaining solution implies that the retailer's bargaining payoff is $\gamma \Pi_{N B}^{*}+(1-\gamma)\left(\Pi_{P L}^{-1 *}-\delta K\right)$ as long as selling the PL product is profitable, e.g., Assumption 2.2 holds.

On the other hand, if the product line (NB, PL) has been chosen at stage one, it means that the retailer has developed its own brand product at stage one and has the ability to sell this product in the event of disagreement. This also yields a positive disagreement payoff to the retailer as long as the condition in Assumption 2.2 holds. As a result, the retailer's bargaining payoff with product line (NB, PL) at stage two is $\gamma \Pi_{N B, P L}^{*}+(1-\gamma) \Pi_{P L}^{*}$, or $\Pi_{P L}^{*}$ since $\Pi_{N B, P L}^{*}=\Pi_{P L}^{*}$ under the conditions given in Lemma 2.2.

The equilibrium profits to the retailer associated different product lines at stage 
two are summarized in Table 2.1 below. $^{6}$

Table 2.1: The equilibrium profit to the retailer at stage two

\begin{tabular}{lccc}
\hline The product line & $(\mathrm{NB}, \mathrm{PL})$ & $(\mathrm{NB}, 0)$ & $(0, \mathrm{PL})$ \\
\hline The Retailer's Profit & $\Pi_{P L}^{*}$ & $\gamma \Pi_{N B}^{*}+(1-\gamma)\left(\Pi_{P L}^{*-1}-\delta K\right)$ & $\Pi_{P L}^{*}$ \\
\hline
\end{tabular}

\subsubsection{The Equilibrium at Stage One in Period 1}

The retailer makes a decision on the product line at this stage. In addition, the retailer incurs a developing cost $K$ in period 1 if the PL product is carried into the product line. Using Table 2.1, we find the equilibrium payoff to the retailer at stage one:

Table 2.2: The equilibrium profit to the retailer at stage one

\begin{tabular}{lccc}
\hline The product line & $(\mathrm{NB}, \mathrm{PL})$ & $(\mathrm{NB}, 0)$ & $(0, \mathrm{PL})$ \\
\hline The Retailer's Profit & $\Pi_{P L}^{*}-K$ & $\gamma \Pi_{N B}^{*}+(1-\gamma)\left(\Pi_{P L}^{*-1}-\delta K\right)$ & $\Pi_{P L}^{*}-K$ \\
\hline
\end{tabular}

Proposition 2.1 Under perfect information, if $c_{N B}^{b} \leq c_{N B}<c_{N B}^{a}$, the sub-game perfect Nash equilibrium (SPNE) is (0, PL).

Proposition 2.1 reveals that in equilibrium the retailer sells only the PL product if the marginal cost of the NB product is very high. Since the retailer does not carry the NB product in equilibrium, it does not need to engage in a negotiation with the NB supplier in this case.

\footnotetext{
${ }^{6}$ Recall that the retailer incurs the developing cost when it introduces the PL product at stage one. Accordingly, such cost does not enter the retailer's profit with product line (NB, PL) and (0, $\mathrm{PL})$ at stage two, but it is a part of the retailer's payoff.
} 


\subsection{Imperfect Information}

In this section, we investigate the equilibrium under imperfect information. As we discussed in section 2.2 , once the PL product is sold and consumed by at least some consumers, its quality becomes known to all the consumers of the same generation in the second period of their lives. However, since information cannot be shared between any two generations, the new consumers do not know the true quality of the PL product when they first enter the market.

The information structure in this OLG model has two implications. First, the new consumers in every period may not fully believe the PL product is manufactured in high quality. Consequently, the retailer has to deal with the problem of asymmetric information in every period. In the retailer's profit maximization problem, therefore, we need to consider its incentive compatibility constraint over all periods. This may affect the retailer's equilibrium pricing strategy and generate additional restrictions on the critical values of the marginal cost for which a particular product line arises in equilibrium.

Second, the information structure among consumers in period 1 is different from that in the following periods. More specifically, none of the consumers in period 1 knows the true quality of the PL product while half of the consumers (i.e., the old generation) in period $t(t \geq 2)$ may know. This difference of information structure may change the retailer's ability to signal the quality of the PL product.

In the following sub-sections, we will study each of the retailer's product line. More specifically, we particularly investigate the case where the cost of the NB product is high such that the retailer does not wish to sell this product accompanied with the PL product under perfect information, i.e., $c_{N B}^{b} \leq c_{N B}<c_{N B}^{a}$.

By backward induction, we start with a discussion about the equilibrium in period 2. Moreover, we will take into account the possibility of a change in product line 
between period 1 and period 2 due to the difference in consumers' beliefs between these two periods. That is, because none of the consumers in period 1 knows the quality of a PL product in period 1 while half of the consumers in period 2 have that knowledge, the retailer may change its product line in period 2 (even though the negotiation between the firms is successful).

\subsubsection{The Equilibrium with the Product Line (0, PL)}

In this sub-section, we analyze the sub-game in which the retailer offers the product line $(0, \mathrm{PL})$. This analysis also enables us to find out the retailers disagreement payoff in its negotiation with the NB supplier in the case where it intends to offer the NB product in conjunction with the PL product.

When the quality of the PL product cannot be observed by the new consumers, the incentive compatibility constraint may cause the equilibrium to differ from that in the benchmark case. Such constraint can be expressed by the inequality $\Pi_{P L}\left(p_{P L}^{1}, p_{P L}^{t}\right) \geq$ $\widetilde{\Pi}_{P L}\left(p_{P L}^{1}, p_{P L}^{t}\right)$, where $\Pi_{P L}\left(p_{P L}^{1}, p_{P L}^{t}\right)$ and $\widetilde{\Pi}_{P L}\left(p_{P L}^{1}, p_{P L}^{t}\right)$ represent the retailer's discounted sum of profits from selling the high-quality and low-quality PL product over all periods, respectively. Moreover, $\widetilde{\Pi}_{P L}\left(p_{P L}^{1}, p_{P L}^{t}\right)$ can be expressed as $\left(p_{P L}^{1}-c_{P L, L}\right) D_{P L}\left(p_{P L}^{1}\right)+$ $\sum_{t=2}^{\infty} \delta^{t-1}\left(p_{P L}^{t}-c_{P L, L}\right) D_{P L}\left(p_{P L}^{t}\right)$. Since the retailer is not allowed to change the product quality once such decision has been made in period 1 , the second term in $\widetilde{\Pi}_{P L}\left(p_{P L}^{1}, p_{P L}^{t}\right)$ implies that the new consumers in every period may purchase from the low-quality retailer since there is no communication between the two generations.

Because the retailer is not permitted to change the product quality once it has been decided in period 1, we are allowed to let the retailer take the incentive compatibility into its first period consideration (i.e., the incentive compatibility constraint holds in the period when the quality decision is made). As a consequence, we can only consider one incentive compatibility constraint in terms of such discounted sum of 
profits. We then construct the retailer's modified profit maximization problem:

$$
\begin{aligned}
& \max \Pi_{P L}\left(p_{P L}^{1}, p_{P L}^{t}\right)=\left(p_{P L}^{1}-c_{P L, H}\right) D_{P L}\left(p_{P L}^{1}\right)+\sum_{t=2}^{\infty}\left(p_{P L}^{t}-c_{P L, H}\right) D_{P L}\left(p_{P L}^{t}\right) \\
& \text { s.t. : } \Pi_{P L}\left(p_{P L}^{1}, p_{P L}^{t}\right) \geq \widetilde{\Pi}_{P L}\left(p_{P L}^{1}, p_{P L}^{t}\right) .
\end{aligned}
$$

Note that in the objective function $\Pi\left(p_{P L}^{1}, p_{P L}^{t}\right)$, the retailer is not allowed to exercise price discrimination between the two generations in any period and thus it serves two generations with the same retail price after period 1. The Lagrangian function associated with the problem in (2.10) can be expressed as:

$$
\mathscr{L}\left(p_{P L}^{1}, p_{P L}^{t}, \lambda\right)=\Pi_{P L}\left(p_{P L}^{1}, p_{P L}^{t}\right)+\lambda\left[\Pi_{P L}\left(p_{P L}^{1}, p_{P L}^{t}\right)-\widetilde{\Pi}_{P L}\left(p_{P L}^{1}, p_{P L}^{t}\right)\right] .
$$

The first-order conditions and the complementary slackness are:

$$
\begin{gathered}
\frac{\partial \mathscr{L}}{\partial p_{P L}^{1}}=(1+\lambda) \frac{\partial \Pi_{P L}\left(p_{P L}^{1}, p_{P L}^{t}\right)}{\partial p_{P L}^{1}}+\lambda \frac{\partial \widetilde{\Pi}_{P L}\left(p_{P L}^{1}, p_{P L}^{t}\right)}{\partial p_{P L}^{1}}=0, \\
\frac{\partial \mathscr{L}}{\partial p_{P L}^{t}}=(1+\lambda) \frac{\partial \Pi_{P L}\left(p_{P L}^{1}, p_{P L}^{t}\right)}{\partial p_{P L}^{t}}+\lambda \frac{\partial \widetilde{\Pi}_{P L}\left(p_{P L}^{1}, p_{P L}^{t}\right)}{\partial p_{P L}^{t}}=0, \\
\Pi_{P L}\left(p_{P L}^{1}, p_{P L}^{t}\right) \geq \widetilde{\Pi}_{P L}\left(p_{P L}^{1}, p_{P L}^{t}\right) ; \quad \lambda \geq 0 ; \quad \lambda\left[\Pi_{P L}\left(p_{P L}^{1}, p_{P L}^{t}\right)-\widetilde{\Pi}_{P L}\left(p_{P L}^{1}, p_{P L}^{t}\right)\right]=0 .
\end{gathered}
$$

By solving the first-order conditions (2.11) and (2.12), we can find the equilibrium retail prices with the product line $(0, \mathrm{PL}): \hat{p}_{P L}^{1}=p_{P L}^{*}+\lambda\left(c_{P L, H}-c_{P L, L}\right) / 2$ and $\hat{p}_{P L}^{t}=p_{P L}^{*}+\lambda\left(c_{P L, H}-c_{P L, L}\right) / 2(2+\lambda)$. Because the demands for the PL product and information structures are not changed from period 2 onwards, the retail prices are the same in every period except period 1.

Moreover, it is easy to verify that $\hat{p}_{P L}^{1} \geq \hat{p}_{P L}^{t} \geq p_{P L}^{*}$ with a non-negative multiplier $\lambda$. This is because that the new consumers in every period do not know the quality of the PL product which causes the retailer to charge a high price (relative to the 
perfect information one) to signal the quality. In addition, after period 1, there is a portion of consumers (i.e., the old generation) who know the actual quality of such product. The retailer can therefore lower the retail price (but still greater than the perfect information one) for the purpose of signaling the quality to the new consumers only.

We study the equilibriums in different cases depending on the cost of the PL product. More specifically, there exists a threshold on $c_{P L, H}$, denoted by $c_{P L, H}^{b}$, below which the retailer charges the PL product at first-best price, i.e., $p_{P L}^{1}=p_{P L}^{t}=p_{P L}^{*}$. Moreover, there is another critical value on $c_{P L, H}$, denoted by $c_{P L, H}^{c}$, such that $c_{P L, H}^{a}>$ $c_{P L, H}^{c}>c_{P L, H}^{b}$ and the retailer charges higher prices for the PL product than those under perfect information, i.e., $\hat{p}_{P L}^{1} \geq \hat{p}_{P L}^{t} \geq p_{P L}^{*}$, if $c_{P L, H}^{b} \leq c_{P L, H}<c_{P L, H}^{c}$.

On the consumer side, we let $S_{N}^{t}=\left\{\alpha_{N}^{t}, \beta_{N}^{t}\right\}$ be the belief system of the new consumers who enter in period $t(t \geq 1)$ and $S_{O}^{t}=\left\{\alpha_{O}^{t}, \beta_{O}^{t}\right\}$ be the belief system of the old consumers. Since the retailer may change its product line in period 2, the belief systems of the new customers who enter in period 2 and onwards may be different from that of new consumers who enter in period $1 .^{7}$ We focus on pure strategy equilibrium only. Consequently, the value of $\alpha$ and $\beta$ can only be "0" or "1".

Note that in this signaling game, the beliefs in set $S_{N}^{t}$ depend on the observed retail prices and the product line (while $S_{O}^{t}$ depends on the old consumers' consumption experience). Thus the (new) consumers will not believe the PL product is of high quality (e.g., $\alpha$ and/or $\beta$ is equal zero) if they observe a price that violates the retailer's incentive compatibility constraint. For example, if the price on the PL product is too low to make the constraint to hold in a given range of costs, consumers' belief about the probability of high quality PL product will be zero. With these in mind, we have

\footnotetext{
${ }^{7}$ This may not be an issue with the product line $(0, \mathrm{PL})$ because the retailer does not wish to change the product line in this case. But that is a matter with the product line (NB, PL) which will be discussed in 4.2 .
} 
following

Lemma 2.3 Under imperfect information, the perfect Bayesian equilibrium (PBE) with the product line $(0, P L)$ is characterized by the following.

(i) In the case where $c_{P L, H}<c_{P L, H}^{b}$, the retailer sets the prices at $p_{P L}^{1}=p_{P L}^{t}=p_{P L}^{*}$. The belief system is $\left\{\alpha_{N}^{1}, \beta_{N}^{1}\right\}=\left\{\alpha_{N}^{t}, \beta_{N}^{t}\right\}=\left\{\alpha_{O}^{t}, \beta_{O}^{t}\right\}=\{1,1\}$ if $p_{P L}^{1} \geq p_{P L}^{*}$ and $p_{P L}^{t} \geq p_{P L}^{*}$, and $\left\{\alpha_{N}^{1}, \beta_{N}^{1}\right\}=\left\{\alpha_{N}^{t}, \beta_{N}^{t}\right\}=\left\{\alpha_{O}^{t}, \beta_{O}^{t}\right\}=\{0,0\}$ otherwise. The retailer's equilibrium profit is $\Pi_{P L}=\Pi_{P L}^{*}$.

(ii) In the case where $c_{P L, H}^{b} \leq c_{P L, H}<c_{P L, H}^{c}$, the retailer sets the prices at $p_{P L}^{1}=\hat{p}_{P L}^{1}$ and $p_{P L}^{t}=\hat{p}_{P L}^{t}$. The belief system is $\left\{\alpha_{N}^{1}, \beta_{N}^{1}\right\}=\left\{\alpha_{N}^{t}, \beta_{N}^{t}\right\}=\left\{\alpha_{O}^{t}, \beta_{O}^{t}\right\}=\{1,1\}$ if $p_{P L}^{1} \geq \hat{p}_{P L}^{1}$ and $p_{P L}^{t} \geq \hat{p}_{P L}^{t}$, and $\left\{\alpha_{N}^{1}, \beta_{N}^{1}\right\}=\left\{\alpha_{N}^{t}, \beta_{N}^{t}\right\}=\left\{\alpha_{O}^{t}, \beta_{O}^{t}\right\}=\{0,0\}$ otherwise. The retailer's equilibrium profit is $\Pi_{P L}=\hat{\Pi}_{P L} \in\left(0, \Pi_{P L}^{*}\right]$.

(iii) In the case where $c_{P L, H}^{c} \leq c_{P L, H}<c_{P L, H}^{a}$, $\Pi_{P L}=0$, there is no PBE with the high-quality $P L$ product in this case.

In addition, $c_{P L, H}^{b}=\delta \theta\left(s_{P L, H}-s_{P L, L}\right) /(2+\delta)+c_{P L, L}, c_{P L, H}^{c} \in\left(c_{P L, H}^{b}, \bar{\theta}\left(s_{P L, H}-s_{P L, L}\right) /(2+\right.$ $\left.c_{P L, L}\right)$.

Lemma 2.3 implies that the retailer's profit from selling product line (0, PL) depends on the cost of the PL product. More specifically, if that cost is low enough, i.e., $c_{P L, H}<c_{P L, H}^{b}$, the retailer will charge perfect information price on the PL product and enjoy the first-best profit in equilibrium. If the cost of the PL product is intermediate, i.e., if $c_{P L, H}^{b} \leq c_{P L, H}<c_{P L, H}^{c}$, the retailer needs to charge higher prices (than that under perfect information) on that product to signal the product is of high quality. Because of these higher retail prices, the retailer's profit from selling the PL product is lower than that in benchmark case, i.e., $\hat{\Pi}_{P L} \leq \Pi_{P L}^{*}$. Moreover, in both case (i) and (ii), consumers believe that the quality of the PL product is high when they observe the associated equilibrium retail prices. 
On the other hand, if such cost is very high, i.e., $c_{P L, H}^{c} \leq c_{P L, H}<c_{P L, H}^{a}$, there is no equilibrium with the sales of the high-quality PL product. This is because the incentive compatibility in the problem (2.10) prevents the retailer from selling the low-quality products and, if the cost of high-quality product is very high, the PBE will not exist in this situation.

One difference here from the two-period model about the product line $(0, \mathrm{PL})$ is that the equilibrium of selling high-quality product may not exist in the OLG model. This is because in the present case, the retailer needs to deal with the problem of asymmetric information with a relatively higher retail price in every period. Accordingly, if the cost of the high-quality PL product is too high, selling such product would not satisfy the retailer's incentive compatibility constraint. In the two-period model, however, the quality of the PL product becomes known to all consumers in period 2 and the retailer can then obtain the first-best profit.

Moreover, as we discussed in 2.3.3.2, another situation of the retailer's disagreement option takes place when it chooses $(\mathrm{NB}, 0)$ in period 1 . That is, the retailer has no chance to sell the PL product in case where the negotiation is failed. Accordingly, the retailer can only sell the PL product from period 2 onwards (by incurring a developing cost $K$ in period 2). In this case, the retailer's disagreement option yields a reduced payoff than that in the above case. Such reduced payoff can be expressed by $\hat{\Pi}_{P L}^{-1}$ which value also varies on $c_{P L, H}$. The value of $\hat{\Pi}_{P L}^{-1}$ is provided in the proof of Lemma 2.3.

\subsubsection{The Equilibrium with the Product Line (NB, PL)}

Given Lemma 2.2, the equilibrium in section 2.3 shows that the retailer does not wish to sell a positive amount of NB products accompanied with the PL product under perfect information because of high cost on the former. In a world of imperfect 
information, however, this result may be changed in three aspects. First, a higher retail price on the PL product (due to imperfect information) may cause the demand for the NB product to become positive. Specifically, the higher price drives down the demand for the PL product, leading some consumers to switch to the NB product. With this in mind, we will examine the possibility that the retailer carries and sells a positive amount of the NB products.

Second, because the problem from asymmetric information arises in every period, the retailer may carry and sell the NB product all the time. Namely, the presence of asymmetric information may cause a higher price on the PL product in a long term, leading the demand for the NB product to be positive in every period.

Third, because there are always some consumers (i.e., the old generation) who know the actual quality of the PL product after period 1, the retailer may charge a relatively lower price on such product than that in period 1 . This lower price may change the retailer's willingness to carry the NB product. In other words, if the retail price on the PL product is low enough after period 1(but still greater than that under perfect information), the retailer may wish to drop the NB product after period 1.

As in 2.3.2., the retailer also maximizes the joint profit under imperfect information. We construct the retailer's profit maximization problem with the product line $(\mathrm{NB}, \mathrm{PL})$ at stage three in period 1 :

$$
\begin{aligned}
& \max \prod_{N B, P L}\left(\mathbf{p}_{N B}, \mathbf{p}_{P L}\right)=\left(p_{N B}^{1}-c_{N B}\right) D_{P L}\left(p_{N B}^{1}, p_{P L}^{1}\right)+\left(p_{P L}^{1}-c_{P L, H}\right) D_{P L}\left(p_{N B}^{1}, p_{P L}^{1}\right) \\
& +\sum_{t=2}^{\infty} 2 \delta^{t-1}\left[\left(p_{N B}^{t}-c_{N B}\right) D_{N B}\left(p_{N B}^{t}, p_{P L}^{t}\right)+\left(p_{P L}^{t}-c_{P L, H}\right) D_{P L}\left(p_{N B}^{t}, p_{P L}^{t}\right)\right] \\
& \text { s.t. : } \prod_{N B, P L}\left(\mathbf{p}_{N B}, \mathbf{p}_{P L}\right) \geq \widetilde{\Pi}_{N B, P L}\left(\mathbf{p}_{N B}, \mathbf{p}_{P L}\right) ; \quad D_{N B}\left(p_{N B}^{1}, p_{P L}^{1}\right) \geq 0 ; \quad D_{N B}\left(p_{N B}^{t}, p_{P L}^{t}\right) \geq 0
\end{aligned}
$$

where $\mathbf{p}_{N B}$ and $\mathbf{p}_{P L}$ respectively denote that the vector of prices on the NB and PL 
product over periods, and the demand function $D_{N B}(\cdot)$ and $D_{P L}(\cdot)$ are given by $(2.3)$ and (2.4), and

$$
\begin{aligned}
\widetilde{\Pi}_{N B, P L}\left(\mathbf{p}_{N B}, \mathbf{p}_{P L}\right)= & \left(p_{N B}^{1}-c_{N B}\right) D_{N B}\left(p_{N B}^{1}, p_{P L}^{1}\right)+\left(p_{P L}^{1}-c_{P L, L}\right) D_{P L}\left(p_{N B}^{1}, p_{P L}^{1}\right) \\
& \sum_{t=2}^{\infty} \delta^{t-1}\left[\left(p_{N B}^{t}-c_{N B}\right) D_{N B}\left(p_{N B}^{t}, p_{P L}^{t}\right)+\left(p_{P L}^{t}-c_{P L, L}\right) D_{P L}\left(p_{N B}^{t}, p_{P L}^{t}\right)\right]
\end{aligned}
$$

represents the retailer's discounted sum of profits from selling the low-quality PL product. Note that the profit $\widetilde{\Pi}(\cdot)$ is dependent on the retail prices in every period. Because the retailer is not permitted to change the product quality once it has been decided in period 1 , the new consumers can still purchase from the low-quality retailer from period 2 onwards. The Lagrangian function to the problem (2.14) is

$$
\begin{aligned}
\mathscr{L}\left(\mathbf{p}_{N B}, \mathbf{p}_{P L}\right)= & \Pi_{N B, P L}\left(\mathbf{p}_{N B}, \mathbf{p}_{P L}\right)+\nu\left[\Pi_{N B, P L}\left(\mathbf{p}_{N B}, \mathbf{p}_{P L}\right)-\widetilde{\Pi}_{N B, P L}\left(\mathbf{p}_{N B}, \mathbf{p}_{P L}\right)\right] \\
& +\xi_{1} D_{N B}\left(p_{N B}^{1}, p_{P L}^{1}\right)+\xi_{2} D_{N B}\left(p_{N B}^{t}, p_{P L}^{t}\right) .
\end{aligned}
$$

Solving the Lagrange function $\mathscr{L}\left(\mathbf{p}_{N B}, \mathbf{p}_{P L}\right)$ yields

$$
\left\{\begin{array} { l } 
{ p _ { N B } ^ { 1 } = p _ { N B } ^ { * } - \xi _ { 1 } / 2 ; } \\
{ p _ { P L } ^ { 1 } = p _ { P L } ^ { * } + \nu ( c _ { P L , H } - c _ { P L , L } ) / 2 , }
\end{array} \quad \text { and } \quad \left\{\begin{array}{l}
p_{N B}^{t}=p_{N B}^{*}-\xi_{2} / 2(2+n u) ; \\
p_{P L}^{t}=p_{P L}^{*}+\nu\left(c_{P L, H}-c_{P L, L}\right) / 2(2+\nu) .
\end{array}\right.\right.
$$

Similar to the case of the product line $(0, \mathrm{PL})$, the retailer charges a price on the PL product that no smaller than the perfect information one in every period. In addition, it can be verified that $p_{P L}^{1} \geq p_{P L}^{t} \geq p_{P L}^{*}$. On the one hand, if the demand for the NB product is zero in every period, i.e., if $\xi_{1} \geq 0$ and $\xi_{2} \geq 0$, the equilibrium with the product line $(\mathrm{NB}, \mathrm{PL})$ will be identical to that with $(0, \mathrm{PL})$. On the other hand, if the demand for the NB product is positive in any period, i.e., if $\xi_{1}=0$ or/and $\xi_{2}=0$, the retailer's equilibrium retail prices will vary on the value of $c$. 
More specifically, when the retailer's incentive compatibility constraint in (2.14) is non-binding, i.e., $\nu=0$, the demand for the NB product cannot be positive in any period. This is because that in this case the retailer charges the PL product at perfect information level, i.e., $p_{P L}^{1}=p_{P L}^{t}=p_{P L}^{*}$, and, given Lemma 2.2, the cost of the NB product is so high that the retailer does not wish to carry it.

However, when the retailer's incentive compatibility constraint is binding, the retail price on the PL product will be increased by a term of $\hat{\nu}$, which can be derived from $\Pi_{N B, P L}\left(\mathbf{p}_{N B}, \mathbf{p}_{P L}\right)-\widetilde{\Pi}_{N B, P L}\left(\mathbf{p}_{N B}, \mathbf{p}_{P L}\right)=0$. In this case, we have following

Lemma 2.4 Under imperfect information, the retailer will carry and sell the NB product if it has done so in period 1, i.e., $D_{N B}\left(p_{N B}^{t}, p_{P L}^{t}\right) \geq 0$ if $D_{N B}\left(p_{N B}^{1}, p_{P L}^{1}\right)>0$.

Lemma 2.4 reveals that the retailer would not sell the NB product if it has not done so in period 1. In fact, the motivation of the retailer to sell the NB product is from a higher retail price on the PL product. Because of a relatively smaller price distortion on such retail price after period one, i.e., $p_{P L}^{t} \leq p_{P L}^{1}$, the retailer will not sell the NB product after period 1 if it has not done so in period 1.

We then study the equilibrium with this product line in different cases according to the cost of the NB product. Specifically, there exists an threshold on $c_{N B}$, denoted by $c_{N B}^{c}>c_{N B}^{b}$, below which the retailer sells both products in every period and, the equilibrium prices are denoted by $p_{N B}^{1}=\hat{\hat{p}}_{N B}^{1}, p_{P L}^{1}=\hat{\hat{p}}_{P L}^{1}$ and $p_{N B}^{t}=\hat{\hat{p}}_{N B}^{t}, p_{P L}^{t}=\hat{\hat{p}}_{P L}^{t}$. Furthermore, there is another threshold on $c_{N B}$, denoted by $c_{N B}^{d} \in\left(c_{N B}^{c}, c_{N B}^{a}\right)$, such that the retailer sells both product only in period 1 if $c_{N B}^{c} \leq c_{N B}<c_{N B}^{d}$ and drops the NB product after period 1. The equilibrium prices in this case are denoted by $p_{N B}^{1}=\hat{\hat{\hat{p}}}_{N B}^{1}<\hat{\hat{p}}_{N B}^{1}, p_{P L}^{1}=\hat{\hat{\hat{p}}}_{P L}^{1}<\hat{\hat{p}}_{P L}^{1}$ and $p_{P L}^{t}=\hat{\hat{\hat{p}}}_{P L}^{t}<\hat{\hat{p}}_{P L}^{t}$ (the value of the prices are also provided in the appendix).Specifically, we have following

Lemma 2.5 Under imperfect information, the perfect Bayesian equilibrium (PBE) with the product line $(N B, P L)$ is characterized by the following. 
(i) In the case where $c_{P L, H}^{b} \leq c_{P L, H}<c_{P L, H}^{c}$ and $c_{N B}^{b} \leq c_{N B}<c_{N B}^{c}$, the retailer sets the price at $p_{N B}^{1}=\hat{\hat{p}}_{N B}^{1}, p_{P L}^{1}=\hat{\hat{p}}_{P L}^{1}$ and $p_{N B}^{t}=\hat{\hat{p}}_{N B}^{t}, p_{P L}^{t}=\hat{\hat{p}}_{P L}^{t}$. The belief system is $\left\{\alpha_{N}^{1}, \beta_{N}^{1}\right\}=\left\{\alpha_{N}^{t}, \beta_{N}^{t}\right\}=\left\{\alpha_{O}^{t}, \beta_{O}^{t}\right\}=\{1,0\}$ if $p_{P L}^{1} \geq \hat{\hat{p}}_{P L}^{1}$ and $p_{P L}^{t} \geq \hat{\hat{p}}_{P L}^{t}$, and $\left\{\alpha_{N}^{1}, \beta_{N}^{1}\right\}=\left\{\alpha_{N}^{t}, \beta_{N}^{t}\right\}=\left\{\alpha_{O}^{t}, \beta_{O}^{t}\right\}=\{0,0\}$ otherwise. The equilibrium (joint) profit is $\Pi_{N B, P L}=\hat{\Pi}_{N B, P L}$ and $D_{N B}^{1}>0, D_{N B}^{t \geq 2}>0$.

(ii) In the case where $c_{P L, H}^{b} \leq c_{P L, H}<c_{P L, H}^{c}$ and $c_{N B}^{c} \leq c_{N B}<c_{N B}^{d}$, the retailer sets the price at $p_{N B}^{1}=\hat{\hat{\hat{p}}}_{N B}^{1}, p_{P L}^{1}=\hat{\hat{\hat{p}}}_{P L}^{1}$ and $p_{P L}^{t}=\hat{\hat{\hat{p}}}_{P L}^{t}$. The belief system is $\left\{\alpha_{N}^{1}, \beta_{N}^{1}\right\}=\{1,0\},\left\{\alpha_{N}^{t}, \beta_{N}^{t}\right\}=\left\{\alpha_{O}^{t}, \beta_{O}^{t}\right\}=\{1,1\}$ and if $p_{P L}^{1} \geq \hat{\hat{\hat{p}}}_{P L}^{1}$ and $p_{P L}^{t} \geq \hat{\hat{\hat{p}}}_{P L}^{t}$, and $\left\{\alpha_{N}^{1}, \beta_{N}^{1}\right\}=\left\{\alpha_{N}^{t}, \beta_{N}^{t}\right\}=\left\{\alpha_{O}^{t}, \beta_{O}^{t}\right\}=\{0,0\}$ otherwise. The equilibrium (joint) profit is $\Pi_{N B, P L}=\hat{\hat{\hat{\Pi}}}_{N B, P L}$ and $D_{N B}^{1}>0, D_{N B}^{t \geq 2}=0$.

(iii) In the case where cplhb $\leq c_{P L, H}<c_{P L, H}^{c}$ and $c_{N B}^{d} \leq c_{N B}<c_{N B}^{a}, \Pi_{N B, P L}=0$, there is no PBE with high-quality PL product in this case.

where $c_{N B}^{c} \in\left(c_{N B}^{b}, \bar{\theta}\left(s_{N B}-2 s_{P L, H}+s_{P L, L}\right)+4 c_{P L, H}-3 c_{P L, L}\right)$, and $c_{N B}^{d} \in\left(\bar{\theta}\left(s_{N B}-\right.\right.$ $\left.\left.2 s_{P L, H}+s_{P L, L}\right)+4 c_{P L, H}-3 c_{P L, L}, c_{N B}^{a}\right)$. In addition, the $\operatorname{set}\left(c_{N B}^{b}, \bar{\theta}\left(s_{N B}-2 s_{P L, H}+s_{P L, L}\right)+\right.$ $\left.4 c_{P L, H}-3 c_{P L, L}\right)$ and $\left(\bar{\theta}\left(s_{N B}-2 s_{P L, H}+s_{P L, L}\right)+4 c_{P L, H}-3 c_{P L, L}, c_{N B}^{a}\right)$ are non-empty if $c_{P L, H}^{b} \leq c_{P L, H}<c_{P L, H}^{c}$.

Lemma 2.5 provides the stage-three equilibrium with the product line (NB, PL) in the case where the retail price on the PL product is upward distorted, i.e., the retailer's incentive compatibility constraint is binding. More specifically, if the cost of the NB product is low enough, i.e., if in case (i), the retailer will sell both PL and NB product in every period. If that cost is higher, i.e., in case (ii), the retailer will sell both products in period 1 only, and, sell only the PL product in all subsequent periods. If the cost of the NB product is even higher, i.e., in case (iii), a diversified product line cannot be part of an equilibrium. The retailer will therefore sell either only NB product or only PL product depending on the profit from the corresponding 
product line (to be discussed later).

The first two cases in Lemma 2.5 indicate that under imperfect information, the retailer may sell a positive amount of NB products in equilibrium although it would not have done so under perfect information. On the surface, this is because the relatively higher retail price on the PL product drives its demand down and causes some PL customers switch to the NB product. Moreover, the presence of the NB product enables the retailer to reduce the upward distortion in the price of the PL product. Indeed, as shown in the proof of Lemma 2.5, the retail price of the PL product is lower in the product line (NB, PL) than that in (0, PL), i.e., $\hat{p}_{P L} \geq \hat{\hat{p}}_{P L} \geq$ $\hat{\hat{\hat{p}}}_{P L}$. This suggests that the(upward) price distortion on the PL product is reduced with the sales of the NB product.

In terms of using the brand stretching strategy, the consumers' belief on the PL product could be "1" in the presence of the NB product, i.e., consumers believe that the PL product is of high quality when they observe the NB product is also offered. This implies that the NB product can play a role to credibly signal the quality of the PL product is high.

Moreover, if $c_{N B}$ (the cost of NB) is relatively high, in equilibrium the retailer sells the NB product in period 1 only, i.e., the retailer changes its product line in period 2. In such a situation, the benefit from selling the NB product becomes smaller after period 1 because the old consumers are informed about the quality of the PL product in each subsequent period. As a result, the retailer stops selling the NB product from period 2 onwards. This result is similar to that in the two-period model which shows that the retailer prefers to sell the NB product in period 1 only. The difference is, in the two-period model, all the consumers in period 2 can find out the true quality of the PL product (due to perfect information dissemination) and the retailer can then earn the first-best profit from selling only the PL product. In the present model, 
however, asymmetric information exists in every period but there is less of need to sell the NB product because the presence of informed (old) consumers after period 1 .

At stage two in period 1, the retailer's bargaining payoff with the product line (NB, PL) is $\gamma \Pi_{N B, P L}+(1-\gamma) \Pi_{P L}$, where both $\Pi_{N B, P L}$ and $\Pi_{P L}$ are determined by the value of $c_{N B}$ and $c_{P L, H}$ derived in Lemma 2.3 and 2.5. Moreover, the retailer's bargaining payoff with the product line $(\mathrm{NB}, 0)$ is $\gamma \Pi_{N B}^{*}+(1-\gamma)\left(\hat{\Pi}_{P L}^{-1}-\delta K\right)$ if it fails to reach an agreement with the NB supplier, where $\hat{\Pi}_{P L}^{-1}$ represents the discounted sum of profits from selling the PL product after period 1 which has been discussed in 2.4.1. Moreover, the value of $\hat{\Pi}_{P L}^{-1}$ under imperfect information is determined by the value of $c_{P L, H}$ which is derived in Lemma 2.3. Specifically, those bargaining payoffs can be summarized in the table below.

Table 2.3: The equilibrium profit to the retailer at stage two in period 1

\begin{tabular}{|c|c|c|c|}
\hline $\begin{array}{l}\text { The Range of } \\
c_{N B} \text { and } c_{P L, H}\end{array}$ & $(\mathrm{NB}, 0)$ & $(0, \mathrm{PL})$ & $(\mathrm{NB}, \mathrm{PL})$ \\
\hline$c_{P L, H}<c_{P L, H}^{b}$ & $\gamma \Pi_{N B}^{*}+(1-\gamma)\left(\Pi_{P L}^{*-1}-\delta K\right)$ & $\Pi_{P L}^{*}$ & $\Pi_{P L}^{*}$ \\
\hline $\begin{array}{c}c_{P L, H}^{b} \leq c_{P L, H}<c_{P L, H}^{c} \\
\text { and } \\
c_{N B}^{b} \leq c_{N B}<c_{N B}^{c}\end{array}$ & $\gamma \Pi_{N B}^{*}+(1-\gamma)\left(\hat{\Pi}_{P L}^{-1}-\delta K\right)$ & $\hat{\Pi}_{P L}$ & $\gamma \hat{\Pi}_{N B, P L}+(1-\gamma) \hat{\Pi}_{P L}$ \\
\hline $\begin{array}{c}c_{P L, H}^{b} \leq c_{P L, H}<c_{P L, H}^{c} \\
\text { and } \\
c_{N B}^{c} \leq c_{N B}<c_{N B}^{d}\end{array}$ & $\gamma \Pi_{N B}^{*}+(1-\gamma)\left(\hat{\Pi}_{P L}^{-1}-\delta K\right)$ & $\hat{\Pi}_{P L}$ & $\hat{\hat{\Pi}}_{N B, P L}+(1-\gamma) \hat{\Pi}_{P L}$ \\
\hline $\begin{array}{c}c_{P L, H}^{b} \leq c_{P L, H}<c_{P L, H}^{c} \\
\text { and } \\
c_{N B}^{d} \leq c_{N B}<c_{N B}^{a}\end{array}$ & $\gamma \Pi_{N B}^{*}+(1-\gamma)\left(\hat{\Pi}_{P L}^{-1}-\delta K\right)$ & $\hat{\Pi}_{P L}$ & 0 \\
\hline$c_{P L, H}^{c} \leq c_{P L, H}<c_{P L, H}^{a}$ & $\gamma \Pi_{N B}^{*}$ & 0 & 0 \\
\hline
\end{tabular}

At stage one in period 1, the retailer makes a decision on its product line. If the PL product is chosen into the product line at this stage, the retailer incurs a developing cost $K$. With the help of Table 2.3, we can derive the PBE under imperfect information. The equilibrium contains the retailer's product line and the correspond- 
ing belief systems of consumers. Again, those beliefs are conditional on retail prices and product line observed by the consumers. The conditions (e.g., the equilibrium prices) have been stated in Lemma 2.3 and 2.5, the stage-two equilibrium profits (to the retailer) have been summarized in Table 2.3 .

Proposition 2.2 Under imperfect information, the perfect Bayesian equilibrium is characterized by the following.

(i) In the case where $c_{P L, H}<c_{P L, H}^{b}$, the product line is ( $\left.0, P L\right)$ and the retailer sets the price at $p_{P L}^{1}=p_{P L}^{t}=p_{P L}^{*}$. The belief system is $\left\{\alpha_{N}^{1}, \beta_{N}^{1}\right\}=\left\{\alpha_{N}^{t}, \beta_{N}^{t}\right\}=$ $\left\{\alpha_{O}^{t}, \beta_{O}^{t}\right\}=\{1,1\}$, if $p_{P L}^{1} \geq p_{P L}^{*}$ and $p_{P L}^{t} \geq p_{P L}^{*}$, and $\left\{\alpha_{N}^{1}, \beta_{N}^{1}\right\}=\left\{\alpha_{N}^{t}, \beta_{N}^{t}\right\}=$ $\left\{\alpha_{O}^{t}, \beta_{O}^{t}\right\}=\{0,0\}$ otherwise. The retailers equilibrium profit is $\Pi_{P_{L}}^{*}-K$.

(ii) In the case where $c_{P L, H}^{b} \leq c_{P L, H}<c_{P L, H}^{c}$ and $c_{N B}^{d} \leq c_{N B}<c_{N B}^{a}$, the product line is $(0, P L)$ and the retailer sets the price at $p_{P L}^{1}=\hat{p}_{P L}^{1}$ and $p_{P L}^{t}=\hat{p}_{P L}^{t}$. The belief system is $\left\{\alpha_{N}^{1}, \beta_{N}^{1}\right\}=\left\{\alpha_{N}^{t}, \beta_{N}^{t}\right\}=\left\{\alpha_{O}^{t}, \beta_{O}^{t}\right\}=\{0,1\}$ if $p_{P L}^{1} \geq \hat{p}_{P L}^{1}$ and $p_{P L}^{t} \geq \hat{p}_{P L}^{t}$, and $\left\{\alpha_{N}^{1}, \beta_{N}^{1}\right\}=\left\{\alpha_{N}^{t}, \beta_{N}^{t}\right\}=\left\{\alpha_{O}^{t}, \beta_{O}^{t}\right\}=\{0,0\}$ otherwise. The retailer's equilibrium profit is $\hat{\Pi}_{P L}-K \leq \Pi_{P L}^{*}-K$.

(iii) In the case where $c_{P L, H}^{b} \leq c_{P L, H}<c_{P L, H}^{c}$ and $c_{N B}^{c} \leq c_{N B}<c_{N B}^{d}$, the product line is $(N B, P L)$ and the retailer sets the price at $p_{N B}^{1}=\hat{\hat{\hat{p}}}_{N B}^{1}, p_{P L}^{1}=\hat{\hat{\hat{p}}}_{P L}^{1}$ and $p_{P L}^{t}=\hat{\hat{\hat{p}}}_{P L}^{t}$. The belief system is $\left\{\alpha_{N}^{1}, \beta_{N}^{1}\right\}=\{1,0\},\left\{\alpha_{N}^{t}, \beta_{N}^{t}\right\}=\left\{\alpha_{O}^{t}, \beta_{O}^{t}\right\}=\{1,1\}$ if $p_{P L}^{1} \geq$ $\hat{\hat{\hat{p}}}_{P L}^{1}$ and $p_{P L}^{t} \geq \hat{\hat{\hat{p}}}_{P L}^{t}$, and $\left\{\alpha_{N}^{1}, \beta_{N}^{1}\right\}=\left\{\alpha_{N}^{t}, \beta_{N}^{t}\right\}=\left\{\alpha_{O}^{t}, \beta_{O}^{t}\right\}=\{0,0\}$ otherwise. The retailer's equilibrium profit is $\gamma \hat{\hat{\Pi}}_{N B, P L}+(1-\gamma) \hat{\Pi}_{P L}-K$ and $D_{N B}^{1}>0$, $D_{N B}^{t \geq 2}=0$.

(iv) In the case where $c_{P L, H}^{b} \leq c_{P L, H}<c_{P L, H}^{c}$ and $c_{N B}^{b} \leq c_{N B}<c_{N B}^{c}$, the product line is $(N B, P L)$ and the retailer sets the price at $p_{N B}^{1}=\hat{\hat{p}}_{N B}^{1}, p_{P L}^{1}=\hat{\hat{p}}_{P L}^{1}$ and $p_{N B}^{t}=\hat{\hat{p}}_{N B}^{t}$, $p_{P L}^{t}=\hat{\hat{p}}_{P L}^{t}$. The belief system is $\left\{\alpha_{N}^{1}, \beta_{N}^{1}\right\}=\left\{\alpha_{N}^{t}, \beta_{N}^{t}\right\}=\left\{\alpha_{O}^{t}, \beta_{O}^{t}\right\}=\{1,0\}$ if $p_{P L}^{1} \geq \hat{\hat{p}}_{P L}^{1}$ and $p_{P L}^{t} \geq \hat{\hat{p}}_{P L}^{t}$, and $\left\{\alpha_{N}^{1}, \beta_{N}^{1}\right\}=\left\{\alpha_{N}^{t}, \beta_{N}^{t}\right\}=\left\{\alpha_{O}^{t}, \beta_{O}^{t}\right\}=\{0,0\}$ 
otherwise. The retailer's equilibrium profit is $\gamma \hat{\hat{\Pi}}_{N B, P L}+(1-\gamma) \hat{\Pi}_{P L}-K$ and $D_{N B}^{1}>0, D_{N B}^{t \geq 2}>0$.

(v) In the case where $c_{P L, H}^{c} \leq c_{P L, H}<c_{P L, H}^{a}$, the product line is $(N B, 0)$ and the retailer sets the price at $p_{N B}^{1}=p_{N B}^{t}=p_{N B}^{*}$. The belief system is $\left\{\alpha_{N}^{1}, \beta_{N}^{1}\right\}=$ $\left\{\alpha_{N}^{t}, \beta_{N}^{t}\right\}=\left\{\alpha_{O}^{t}, \beta_{O}^{t}\right\}=\{0,0\}$ for all $p_{P L}^{1}$ and $p_{P L}^{t}$. The retailer's equilibrium profit is $\gamma \Pi_{N B}^{*}$.

Proposition 2.2 implies that the retailer's equilibrium product line crucially depends on the marginal costs of the NB and PL products. Specifically, if the cost of the PL product is small enough, i.e., in case (i), the retailer will sell only the PL product in equilibrium. If the cost advantage of the PL product is smaller, i.e., if in case (ii), the retailer will still sell only the PL products but the profit is reduced than that in situation (i) since $\hat{\Pi}_{P L} \leq \Pi_{P L}^{*}$. The difference between case (i) and (ii) is that in the latter the retailer needs to charge a relatively higher retail price on the PL product to signal such product is of high quality. The higher retail price reduces the equilibrium profit to be lower than that under perfect information.

If the cost advantage of the PL product is even smaller, i.e., in case (iii) and (iv), the retailer will carry a positive NB products into the product line. Note that with the presence of the NB product, the consumers' belief on the quality of the PL product is one. As we shown in Lemma 2.5, the presence of the NB product enables the retailer to reduce the retail price of the PL product. Such a lower price would not signal the PL product is of high quality if the NB product were absent in the product line, i.e., consumers' belief on the quality of the PL product in $(0, \mathrm{PL})$ would be " 0 " with such a low price. Therefore, the sales of the NB product in the two cases can be viewed as a brand stretching strategy with which the retailer is able to use the NB product to signal the quality of the PL product.

The difference between case (iii) and case (iv) is the length of time during which 
such strategy is used. More specifically, given that the old consumers know the quality of the PL product from period 2 onwards, there is no longer a need to signal its quality after period 1 if the cost advantage of the PL product is relatively large, i.e., in case (iii).

Finally, if the cost of the PL product is very high, as in situation (v), the retailer will sell only the NB product in equilibrium because there is no PBE with the product line $(0, \mathrm{PL})$ or $(\mathrm{NB}, \mathrm{PL})$.

A difference between the results here and those in the two-period model is that the retailer may sell the NB product accompanied with the PL product in a long term under OLG framework. This reveals that the impact of the consumers' beliefs on the product quality with different product line (as well as observed prices in equilibrium). That is, asymmetric information arises from the new generation in every period, thus the retailer may have incentive to signal the quality of its own brand product is of high quality with selling the NB product, i.e., use brand stretching. This enables us to provide an explanation to the observation that the retailer sells the NB product not only in the period when it initially introduces its own brand product (but also in subsequent periods).

Similar to the two-period model, the retailer in the present model may sell the NB product in period 1 only. In the former model, the role of the NB product can be viewed a signal on the quality of the PL product in period 1. And due to perfect information dissemination, the retailer can earn the first-best profit from selling only the PL product in period 2. In the latter model, however, although asymmetric information still presents after period 1, there is less of a need to signal such quality after period 1 because the product quality can be known by the old consumers. Accordingly, for some ranges of costs $\left(c_{N B}\right.$ and $\left.c_{P L, H}\right)$, the retailer will not offer such product after period 1 even though it cannot obtain a first-best profit from selling 
only the PL product.

\subsection{Conclusion}

We have constructed and studied an overlapping-generation model of the private label product under both perfect and imperfect information about the product quality. In the benchmark case of perfect information, we have shown that in the case where the cost of the national brand product is high, the retailer does not wish to carry and sell such product in equilibrium.

In an environment of asymmetric information where the quality of the private label product cannot be observed by the consumers before they purchase, we have first found that the retailer cannot always benefit from launching the private label product if the cost of such product is very high. This is because that asymmetric information causes higher price on the private label product in every period. Accordingly, the demand for such product will be driven down to zero if its cost exceeds a certain value.

In addition, we have shown that the retailer may sell a positive amount of national brand product accompanied with the private label product in the case where it would have not done so under perfect information. There are several implications from this result. First, it implies that the sales of such branded product can be viewed as a credible signal on the quality of the private label product, i.e., brand stretching.

Second, given that there are new consumers enter the market in every period under the overlapping-generation framework, if the cost advantage of the private label product (with respect to that of the national brand product) is not very large, the retailer will use the brand stretching strategy in every period. This explains that the retailer's motivation of selling the national brand product (accompanied with the private label) in a long term under imperfect information. 
Finally, because the old consumer can know the product quality in period 2 onwards, the retailer has stronger incentive to signal such quality in period 1 . If the cost advantage of the private label product is large, the retailer will use the brand stretching strategy in period 1 only.

Moreover, the conditions in equilibrium we have discussed may change when some assumptions on consumers are modified. More specifically, there are two main modifications that are worthwhile to be mentioned here. (1) The information about product quality is partially disseminated between the generations. (2) The size of cohort is unbalanced (i.e., different cohorts have different sizes). Although two distinct modifications, both of them will affect the retailer's ability of using the brand stretching strategy. For instance, if some new consumers can also know the quality of the private label product (or the size of new cohort is smaller than its old counterpart), the retailer will have less incentive to signal such quality. But, the retailer still needs to do so as long as asymmetric information exists. Further, if the fraction of informed consumers approaches zero (or the size of one cohort is close to the size of the other

cohort), we may still obtain the same results as the present study by continuity. Moreover, if the information is perfectly disseminated between the two generations, the results will be the same as that in the basic two-period model. The model presented in this chapter simply eases the difficulties from relaxing those assumptions and, does not deviate from the rationale for the use of brand stretching by a retailer with the private label product.

\section{Bibliography}

Belleflamme, P. And M. Peitz (2010): Industrial Organization: Markets and Strategies, Cambridge: Cambridge University Press. 
Bergès-Sennou, F. (2006): "Store Loyalty, Bargaining Power and the Private Label Production Issue," European Review of Agricultural Economics, 33, 315-335.

Gabrielsen, T. S. and L. Sorgard (2007): "Private Labels, Price Rivalry, and Public Policy," European Economic Review, 51, 403-424.

Harsanyi, J. And R. Selten (1972): "A Generalized Nash Solution for Twoperson Bargaining Games with Incomplete Information," Management Science, 18, 80-106.

Mills, D. E. (1999): "Private Labels and Manufacturer Counterstrategies," European Review of Agriculatural Economics, 26, 125-145.

Mussa, M. And S. Rosen (1978): "Monopoly and Product Quality," Review of Industrial Organization, 18, 301-017.

Narasimhan, C. And R. T. Wilcox (1998): "Private Labels and the Channel Relationship: A Cross-Category Analysis," The Journal of Business, 71, 573-600.

Spengler, J. J. (1950): "Vertical Integration and Antitrust Policy," Journal of Political Economy, 58, 347-352.

Tirole, J. (1988): The Theory of Industrial Organization, The MIT Press. 


\section{Chapter 3}

\section{Private Labels: Bargaining Power and Strategic Retail Pricing}

\subsection{Introduction}

Private labels (PLs) play a significant role for consumer goods in many categories. Examples include, canned products, pasta, cereals, sodas, detergents, and other household items. According to the Nielsen Company, sales of PL food products in the U.S. increased from $\$ 95$ billion in 2011 to $\$ 113$ billion by 2014. A report from the Agriculture and Agri-Food Canada also shows that PL introduction has increased substantially between 2004 and 2009 (Agriculture and Agri-Food Canada, 2013).

Given the increased importance of PLs in retailing, it is not surprising that a number of papers have studied the firms' incentives to introduce PLs. ${ }^{1}$ In particular, these studies focus on 1) how the introduction of PLs can increase a retailer's profit by segmenting the market and facilitating price discrimination among consumers with heterogeneous tastes (e.g., Narasimhan and Wilcox, 1998 and Bergès-Sennou, 2006); 2) how the introduction of PLs can enhance a retailer's bargaining position against

\footnotetext{
${ }^{1}$ See Bergès-Sennou et al. (2004) for a survey.
} 
its NB manufacturers with respect to the wholesale prices (e.g., Mills, 1995; Bontems et al., 1999 and Gabrielsen and Sorgard, 2007). First, with the launch of the PL product, a substitute for the national brand (NB) product, the retailer is able to create an environment of product differentiation to serve heterogeneous consumers. In addition, with the possibility that some NB customers switch to the PL, the NB manufacturer may wish to lower the price of its brand to better compete with the PL. This, in turn, could mitigate the effect of double-marginalization in the pricing of the NB.

Apart from theoretical studies, empirical studies have tried to disentangle the effect of PLs on retailer's prices and profits. These papers can be summarized into the following three groups. First, some of them point that the margins provided by PLs are higher than that by NBs (e.g., Hoch and Banerji, 1993; Raju et al., 1995 and Ailawadi and Harlam, 2004). Specifically, relatively lower wholesale prices on PLs lead to competitive retail prices on the associated products relative to their NB counterparts. Such prices help PL products to better capture market share in the category, leading to higher percentage margins for the retailer.

Second, a branch of empirical studies highlights the retailer's benefit from setting the retail marketing mix in the presence of PLs. (e.g., Chintagunta, 2002; Sayman et al., 2002 and Pauwels and Srinivasan, 2004). The main results from these studies provide empirical evidence to support the findings from the theoretical papers about market segmentation with PL products.

Third, the PLs that are imitations of NBs enable the retailer to obtain better terms in negotiations of prices (Bontemps et al., 2008 and Meza and Sudhir, 2010). This is because the imitating PLs strengthen the retailer's bargaining power against manufacturers selling the imitated NB products.

The ability of the retailer to affect wholesale prices and to set its retail prices in the 
presence of PLs, however, have not been widely discussed in the empirical analyses cited above. To my knowledge, Meza and Sudhir (2010) is the only empirical study on these two issues. They find that when PL products are introduced by the retailer, NB manufacturers lower their prices for their imitated products. Furthermore, the retailer strategically supports their own brand products by keeping the retail prices of those imitated NB products at high levels. However, they do not consider the effects of those PL products that are not imitations of the NB. As opposed to the imitating PLs, the non-imitating ones do not seek to mimic the packaging, design and appearance of the NBs.

In the spirit of the existing empirical studies, the objective of this study is therefore to test whether PLs can help the retailer obtain lower wholesale prices from NB manufacturers - this is the bargaining power hypothesis - and, whether the retailer has an incentive to strategically set retail prices to favor the PLs - this is the strategic retail pricing hypothesis. I also use a simulation exercise to evaluate the benefit of PLs to the consumers.

In particular, I test the two hypotheses inserting a term into the associated profit maximization problems. This term measures the departure of the observed margins from the predicted levels (Chintagunta, 2002; Villas-Boas and Zhao, 2005 and Meza and Sudhir, 2010). Meza and Sudhir (2010) and Villas-Boas and Zhao (2005) refer to this term as a deviation of the observed margins from their predicted counterparts.

Specifically, using an econometric model, I first calculate such deviations from the observed and predicted margins, then regress the deviations on the associated PL variables to evaluate the effect of the launch of PLs on both retail and wholesale prices. To understand the deviations, I start with the retail side. In order to better help its PL products to penetrate the market, the retailer may tend to charge relatively higher prices on NB products to steer consumers to their PL substitutes. The 
manufacturers may also react to such a pricing behavior by the retailer with lower wholesale prices. Specifically, when strategic retail pricing disfavors the market share of the NB products, their manufacturers will tend to lower the wholesale price on such products to prevent their market shares from falling via a pass-through mechanism. The difference between the observed prices in the data and the predicted ones resulting from the retailer and manufacturers' behavior can be captured by the deviations mentioned above.

Following Meza and Sudhir (2010), I measure such deviations in both the retailer and manufacturers' profit maximization problems using the difference between their observed and predicted margins. For my empirical application, I use data on cereals for Dominick's Finer Foods, a large super-market chain in the Chicago area, from 1989 to 1997. My empirical strategy for testing the bargaining power and strategic retail pricing hypotheses controls for the effect of the changed market structure on wholesale and retail prices due to the presence of PLs using a structural approach.

In terms of methodology, I begin by estimating a logit demand model for the cereal brands offered by Dominick's. ${ }^{2}$ Given the demand estimates, I solve for the retail and wholesale predicted margins assuming the retailer-manufacturers two-stage sequential model by Sudhir (2001). I then compute the differences between the observed margins and the margins implied by the model and analyze how these differences are related to the number of PLs offered by Dominick's using a regression approach. Finally, I use a simple back-of-envelope (BOE) exercise to measure the benefit of PLs to the consumers following Hausman and Leonard (2002). For this purpose, I first construct a counterfactual scenario, where the retailer offers no PLs. I then calculate the consumer surplus with and without the PLs to evaluate the welfare effects of the PL introduction.

\footnotetext{
${ }^{2}$ I employ the logit demand model here following Berry (1994).
} 
Broadly speaking, this chapter makes three contributions to the empirical literature on PL products. First, I evaluate how the launch of PLs affects the retailer's ability to lower the prices from its NB manufacturers. I find that the observed wholesale prices of NB products are lowered below their predicted levels when the retailer offers a larger number of PLs in support of the bargaining power hypothesis.

Second, by testing the strategic retail pricing hypothesis, I find that as the number of PLs increases, the observed retail prices of NB products increase above their predicted levels, which implies that the retailer wishes to favor its own brand products over NB products by rising the prices of the latter.

According to my results, there is an overall decrease (increase) on wholesale prices (retail prices) of NB products when the number of PLs increases. These changes take place in both imitated and non-imitated NB products, suggesting that the retailer's ability of affecting NB prices in the presence of PLs is not only limited to those imitated products, but also non-imitated products. Moreover, the motivation of the retailer to strategically increase the prices of NB products affects both imitated and non-imitated groups.

Finally, by simulating a counterfactual situation where PLs are not offered by the retailer, I find that consumers benefited substantially from the introduction of PL products. The benefit to consumers in this study is mostly focused on the improvement on the retailer's product line, i.e., the increase in the variety of brands.

The rest of the chapter is organized as follows. Section 3.2 describes the model. In Section 3.3, I discuss the estimation strategy. In Section 3.4, I present the result of my empirical analysis including the simulation exercise to evaluate the consumer benefits from the PLs. Section 3.5 concludes. 


\subsection{Model}

The objective of my empirical exercise is to test the two hypotheses mentioned in Section 3.1 by measuring the differences between the observed and predicted retail and the wholesale margins while accounting for the change in market structure due to the presence of PLs. To achieve this objective, I first develop a demand model. I then use my demand model estimates to calculate the predicted retail and wholesale margins assuming a two-stage game in a vertical chain that consists of a retailer and several manufacturers. Finally, I assess departures (i.e., deviations) from predicted margins by comparing the observed margins in the data with the ones implied by my model.

\subsubsection{Demand Side}

Given that my application uses the cereal category that has a large number of brands, a parsimonious demand system is necessary. For this reason, I use a logit demand model as in Berry (1994), where the utility of a product is modeled as a function of a few characteristics and price. In the case of cereals, examples of such characteristics are nutrition facts and sugar content. ${ }^{3}$ In addition, due to the fact that Dominick's stores are grouped into four price zones catering to different consumer demographics, and the stores' pricing and promotion strategies are the same in each price zone, I specify the demand model at the price zone level. ${ }^{4}$

Following the approach in Berry, the conditional indirect utility of consumer $i$ from brand $j$ in zone $z$ and time $t$ is

$$
u_{i j z t}=x_{j} \beta-\alpha p_{j z t}+\xi_{j z t}+\varepsilon_{i j z t},
$$

\footnotetext{
${ }^{3}$ Figure 3.1 gives an example of the nutrition facts and sugar content of Frosted Flakes by Kellogg's.

${ }^{4}$ More details about Dominick's price zones will be discussed later.
} 


$$
i=1, \ldots, N, j=1, \ldots, J, z=1, \ldots, Z, t=1, \ldots, T \text {. }
$$

In terms of notation, $p_{j z t}$ denotes the price and $x_{j}$ is a vector of non-price characteristics. The coefficients $\beta$ capture consumers' tastes for the brand characteristics, and $\alpha$ captures the consumers' distaste for price. The aggregate demand shock $\xi_{j z t}$ plays the role of the structural demand shock. It includes the unobserved - to the econometrician - features of the product, such as quality and brand equity that affect demand. The presence of $\xi_{j z t}$ implies endogeneity of prices because both consumers and firms observe $\xi$ and, hence, its value enters the firms' pricing decisions. Finally, $\varepsilon_{i j z t}$ is a mean-zero iid demand shock that captures consumer heterogeneity and it is distributed according to a Type I extreme-value distribution. As it is shown below (in equation (3.2)), this stochastic term will be integrated out and it will not serve as a source of econometric uncertainty.

I also define the mean utility for a brand that is common across all consumers, which will play an important role for my derivations below, using the following

$$
\delta_{j z t}=x_{j} \beta-\alpha p_{j z t}+\xi_{z j t}
$$

Following the standard approach in the literature, it is possible for consumers to purchase none of the $J$ available brands; that is, I treat no purchases as the "outside good" with mean utility $\delta_{0 z t}=0$. Hence, I allow consumers to not purchase at all if they find cereal prices too high. In the absence of the outside good, consumers are forced to choose from the "inside goods" and demand depends only on differences in prices. Therefore, a general increase in prices will not decrease aggregate output. Simply put, without the outside good, it is not possible to calculate an aggregate price elasticity.

A consumer chooses brand $j$ that maximizes her utility, $u_{i j z t} \geq u_{i k z t}$, for all 
$k \neq j$. Given the distributional assumptions regarding consumer heterogeneity, the probability that consumer $i$ chooses brand $j$ in zone $z$ and time $t$ is given by

$$
P_{i j z t}\left(\delta_{j z t}, \varepsilon_{i j z t}\right)=\frac{\exp \left(\delta_{j z t}+\varepsilon_{i j z t}\right)}{\sum_{k=0}^{J} \exp \left(\delta_{k z t}+\varepsilon_{i k z t}\right)} .
$$

Using $A_{j z t}\left(=\left\{\varepsilon_{i 0 z t}, \ldots, \varepsilon_{i J z t} \mid u_{i j z t}>u_{i l z t}, \forall l=0,1, \ldots, J\right\}\right.$ to denote the set of consumers choosing brand $j$ in zone $z$ and market $t$, the market share of brand $j$ is just the integral of consumers in the region $A_{j z t}$

$$
s_{j z t}\left(\delta_{j z t}\right)=\int_{A_{j z t}} P_{i j z t}\left(\delta_{j z t}, \varepsilon_{i j z t}\right)=\frac{\exp \left(\delta_{j z t}\right)}{\sum_{k=0}^{J} \exp \left(\delta_{k z t}\right)} .
$$

The demand $q_{j z t}$ is obtained by multiplying the market share in (3.4) by the total potential market $M_{z t}$ size in each zone

$$
q_{j z t}=M_{z t} s_{j z t}\left(\delta_{j z t}\right)
$$

The own and cross price elasticities of the market shares are then given by

$$
\begin{aligned}
& \frac{\partial s_{j z t}}{\partial p_{j z t}}=-\alpha s_{j z t}\left(1-s_{j z t}\right) \\
& \frac{\partial s_{j z t}}{\partial p_{k z t}}=\alpha s_{k z t} s_{j z t} .
\end{aligned}
$$

\subsubsection{Supply Side}

On the supply side, I consider a two-stage game between manufacturers and a retailer. As illustrated in Figure 3.2, upstream cereal manufacturers compete in a static Bertrand game and simultaneously set wholesale prices in the first stage of the model. Having observed the set of wholesale prices, the retailer sets prices in the second stage of the model. One-sided arrows represent sequential decisions between 
manufacturers and the retailer, while double-sided arrows indicate simultaneous decisions among manufacturers. Assuming this setup, I proceed using backward induction solving first the retailer pricing model.

\subsubsection{Retail Pricing}

Given that the retailer sets the price for a brand $j$ in a price zone $z$ at time $t$, its profit function is given by

$$
\Pi_{z t}=\sum_{j=1}^{J}\left(p_{j z t}-w_{j z t}\right) s_{j z t} M_{z t}
$$

where $p$ and $w$ denote the retail and wholesale price, respectively. In addition, $s$ denotes the market share and $M$ denotes the total market size that accounts for the possibility of no purchasing. Assuming that the consumers of each pricing zone do not shop outside their zone, the first-order conditions with respect to the retail prices in each zone are of the form

$$
s_{j z t}+\sum_{k=1}^{J}\left(p_{k z t}-w_{k z t}\right) \frac{\partial s_{k z t}}{\partial p_{j z t}}=0
$$

Solving the system of $J$ simultaneous equations in (3.9), the retailer's equilibrium prices can be expressed in matrix form as follows

$$
p_{z t}=w_{z t}-\left(\frac{\partial s_{z t}}{\partial p_{z t}}\right)^{-1} s_{z t}
$$

where $\partial s_{z t} / \partial p_{z t}$ is of dimensions $J \times J$ and contains the derivatives of market shares with respect to prices. In order to evaluate the potential deviations from the predicted 
margin, I consider an "as if" objective function for the retailer of the form

$$
\Pi_{z t}=\sum_{j=1}^{J}\left[\left(p_{j z t}-w_{j z t}\right) s_{j z t}-\phi_{j z t}^{R} s_{j z t}\right] M_{z t}
$$

The additional term $\phi_{j z t}^{R}$ in (3.11) compared to (3.8) implies that the retailer may be able to favor (disfavor) the brand $j$ by imposing a negative (positive) weight on the associate market share, $\phi_{j z t}$, which needs to be estimated. ${ }^{5}$ Given the modified objective function, the retail pricing equation in matrix form can be written as follows assuming wholesale prices are available

$$
\underbrace{p_{z t}-w_{z t}}_{\text {observed margin }}=\underbrace{-\left(\frac{\partial s_{z t}}{\partial p_{z t}}\right)^{-1} s_{z t}}_{\text {predicted margin }}+\underbrace{\phi_{z t}^{R}}_{\text {deviation }} .
$$

In equation $(3.12), \phi_{z t}^{R}$ captures the deviations of the observed prices from the data from the predicted retail ones implied by the model. Hence, a positive $\phi_{z t}^{R}$ implies a higher price relative to the predicted price in order to disfavor the brand. I then parameterize deviations in terms of a set of variables $d_{j z t}^{R}$ such that

$$
\phi_{j z t}^{R}=d_{j z t}^{R}+\varepsilon_{j z t}^{R} \quad \varepsilon_{j z t}^{R} \sim N\left(0, \sigma^{2}\right) .
$$

The variables in $d_{j z t}^{R}$ not only control for the presence of PLs, but also control for other brand and promotions effects, such as the strategy the retailer uses when it offers PLs - for example, whether the PL product imitates the existing NB product. Accordingly, $d_{j z t}^{R}$ includes the number of PLs offered by the retailer, promotion variables, imitation variable, wholesaler fixed effect as well as the retailer's price zone fixed effect.

\footnotetext{
${ }^{5}$ Chintagunta (2002) points out that this term represents the importance of store brand market share relative to the importance of category profits.
} 


\subsubsection{Manufacture Pricing}

The cereal manufacturers play a Bertrand game and sell their brands through Dominick's retail chain. Assuming $F$ manufacturers each selling cereal brands in the set $J_{f}$ at time $t$, the objective function of manufacturer $f$ is

$$
\Pi_{t}^{f}=\sum_{j=1}^{J_{f}}\left(w_{j t}-c_{j t}\right) q_{j t}
$$

where $q_{j t}=\sum_{z=1}^{Z} s_{z j t} M_{z t}$ and $c_{j t}$ is the manufacturer's marginal cost. Note also that the wholesale prices do not exhibit variation by zone. Solving for the manufacturers' first-order conditions with respect to the wholesale prices, we obtain the following using matrix notation

$$
w_{t}=c_{t}+\left[\left(-\frac{\partial q_{t}}{\partial w_{t}}\right) \cdot *(\Omega)\right]^{-1} q_{t}=c_{t}+m g_{t}^{M}
$$

where $w_{t}, c_{t}$ and $q_{t}$ are $J \times 1$ vectors, and $\Omega$ is a $J \times J$ ownership matrix whose elements are given by

$$
\Omega(j, r)= \begin{cases}1, & \text { if }\{j, r\} \in J_{f} \\ 0, & \text { otherwise }\end{cases}
$$

Since the retailer sells quantity $q_{z t}$ in each zone (recall that there are four price zones), we can write

$$
q_{t}=\sum_{z=1}^{4} s_{z t} M_{z t} \quad \frac{\partial q_{t}}{\partial w_{t}}=\sum_{z=1}^{4} \frac{\partial s_{z t}}{\partial w_{t}} M_{z t}
$$


Note also that the following holds due to the chain rule

$$
\frac{\partial s_{z t}}{\partial w_{t}}=\frac{\partial s_{z t}}{\partial p_{z t}} \cdot \frac{\partial p_{z t}}{\partial w_{t}}
$$

In terms of notation, $\frac{\partial s_{z t}}{\partial p_{z t}}$ is the familiar $J \times J$ matrix of retailer's zone-specific price derivatives of demand, and $\frac{\partial p_{z t}}{\partial w_{t}}$ is the $J \times J$ matrix of zone-specific pass-through terms. The pass-through terms are obtained using the implicit function theorem in the retailer's first-order conditions. The details about the pass-through terms are provided in the Appendix.

Similar to the retailer deviations, I also allow for deviations from the predicted prices of the manufacturer using also an "as if" profit function that puts a weight on the market share. I then obtain the following manufacturer pricing equation in matrix form

$$
w_{t}=\underbrace{c_{t}}_{\text {cost }}+\underbrace{m g_{t}^{M}}_{\text {predicted margin }}+\underbrace{\phi_{t}^{M}}_{\text {deviation }} .
$$

I further parametrize the deviations $\phi_{j z t}^{M}$ in terms of a set of variables $d_{j z t}^{M}$ (along the lines of that in (3.13)) that account for the presence of PLs but also control for other brand and promotion effect on prices

$$
\phi_{j z t}^{M}=d_{j z t}^{M}+\varepsilon_{j z t}^{M} \quad \varepsilon_{j z t}^{M} \sim N\left(0, \sigma^{2}\right)
$$

\subsection{Estimation}

The model is estimated in two steps. In the first step, I estimate the demand model. In the second step, conditional on the demand estimates, the firms' profit-maximizing pricing equations can be estimated. I start by noting that the demand model can be 
written as

$$
\ln \left(s_{j z t}\right)-\ln \left(s_{0 z t}\right)=\delta_{j z t}=x_{j} \beta-\alpha p_{j z t}+\xi_{z j t} .
$$

To estimate the demand model in (3.21), I use a two-stage least squares (2SLS) approach to address the endogeneity of price. Additionally, in the spirit of Nevo (2001), I use brand-specific dummies instead of detailed product characteristics. There are two main reasons behind this decision. The first is data availability. My sample contains PL brands from Dominick's for which product characteristics are not available given that my sample stops in 1997 . The second is to alleviate somewhat the need to instrument for the correlation between prices and the unobserved quality of the product $\xi_{j z t}$. A brand dummy $\xi_{j}$ allows me to capture some components of the unobserved characteristics that do not vary by price zone and time. Therefore, the correlation between prices and brand-specific means of unobserved quality is accounted for.

To construct valid instruments that address any remaining concerns about price endogeneity, I follow one of the main approaches in the literature and assume that product characteristics are predetermined and are not adjusted by the manufacturers in response to the demand shocks (Berry et al., 1995). Given this assumption, characteristics of other brands will be correlated with price, since the price markups are functions of characteristics based on the profit-maximization first order conditions. Previous papers have suggested using sums or averages of the characteristics of other products produced by the same firm or products produced by rival firms as instruments in many industries, including cereals (Hausman, 1996 and Nevo, 2000). With this in mind and given that I only use brand-specific dummies as opposed to product characteristics, I construct the following instruments: the number of other brands offered by the same cereal manufacturer and the number of brands offered by the rival cereal manufacturers. 
In the second step, with the parameters from the demand estimation, I can estimate the supply side parameters as follows. First, I compute both retail and manufacturer predicted margins conditional on the derivatives of shares with respect to prices, which are a function of the demand parameters

$$
m g_{j z t}^{R}\left(w_{j z t}, s_{j z t}, s_{t}^{*}\right) \text { and } m g_{j z t}^{M}\left(s_{j z t}, s_{t}^{*}\right)
$$

where $s_{t}^{*}$ represents the partial derivatives of shares with respect to prices.

Using (3.12) and (3.13), I compute $\phi_{j z t}^{R}$ as the difference between the observed retail margin $M G_{j z t}^{R}$ and the predicted margin $m g_{j z t}^{R}$ implied by the model

$$
\phi_{j z t}^{R}=M G_{j z t}^{R}-m g_{j z t}^{R}=d_{j z t}^{R}+\varepsilon_{j z t}^{R} .
$$

Similarly, Using (3.19) and (3.20), I compute $\phi_{j z t}^{M}$ as the difference between the manufacturer margin $M G_{j z t}^{M}=w_{j t}-c_{j t}$ and the predicted margin $m g_{j z t}^{M}$

$$
\phi_{j z t}^{M}=M G_{j z t}^{M}-m g_{j z t}^{M}=d_{j z t}^{M}+\varepsilon_{j z t}^{M} .
$$

I then estimate (3.23) and (3.24) using OLS and treating $d_{j z t}^{R}$ and $d_{j z t}^{M}$ as a set of variables of interest mentioned in Section 3.2. The estimation of equation (3.24), however, is complicated by the fact that I do not observe manufacturer margins. Instead, I only observe wholesale prices, which leaves manufacturer cost to be estimated along with the deviations. To be able to separately identify manufacturer costs from the deviations, I assume that manufacturer costs exhibit variation only by manufacturer and time. 


\subsection{Empirical Analysis}

\subsubsection{Preliminaries}

I use publicly available data for the ready-to-eat cereal category in the Dominick's Database at the University of Chicago Booth School of Business. ${ }^{6}$ As a subsidiary of Safeway Inc., Dominick's Finer Foods (DFF) is Chicago's second-largest supermarket operation, trailing only the Jewel-Osco during the sample period. ${ }^{7}$ Other main competitors include ALDI, Save-A-Lot, Mariano's, Whole Foods and Cub Foods. ${ }^{8}$ In addition, Dominick's divides its stores into four price zones: High, Medium, Low and Cub-fighter. The stores designated as "Cub-fighter" are located close to a Cub Foods store and thus compete directly with it. ${ }^{9}$ The other three price zones employ a similar consumer demographics and responsiveness to marketing mix.

The Dominick's data used in this chapter cover cereal sales for roughly 80 stores in four pricing zones form September 1989 to May 1997. The cereal category is well suited for my empirical exercise for, at least, two reasons. First, during this period, Dominick's not only introduced PLs, some of which aimed to imitate existing NBs, but also discontinued some of its existing PLs. ${ }^{10}$ Second, cereals are an economically important category to the retailer, which suggests that strategic behavior is likely for this category.

To keep the number of NBs manageable and taking into account data availability,

\footnotetext{
${ }^{6}$ See http://research.chicagobooth.edu/kilts/marketing-databases/dominicks/ dataset. Note that data are not available for 1995Q2.

${ }^{7}$ By the end of 2007, Dominick's market share in the Chicago region was 15 percent and the Jewel-Osco was 45 percent (Schmeltzer, 2007).

${ }^{8} \mathrm{~A}$ detailed summary about grocery stores in Chicago area is discussed "Chicago's Shifting Grocery Landscape Mirrors Changing City Economics", August, 2014, https ://www.wbez.org/shows/ wbez-news/chicagos-shifting-grocery-landscape-mirrors-changing-city-economics/ ede9578d-87e4-4d65-b09c-a16dc1a4408f.

${ }^{9} \mathrm{Cub}$ Foods is a supermarket chain with seventy-seven stores in Minnesota and Illinois. The company is a wholly owned subsidiary of Eden Prairie, Minnesota-based SuperValu Inc.

${ }^{10}$ Following Meza and Sudhir (2010), I use the names of the PLs to identify which NBs are imitated. Usually, Dominick's names its own brands in the same or similar ones as the NBs.
} 
I use the top 20 NBs that account for $40 \%$ of total dollar sales in the category listed in Table 3.1. Six of these NBs, all manufactured by Kellogg's, have been imitated by Dominick's PLs, and are indicated by an asterisk in the table. I also aggregate the data by brand, such that different size boxes under one brand name are considered one brand. For this reason, I report quantities in oz. and prices $\$ /$ oz. In addition, I use the retail margin data readily available from Dominick's to compute wholesale prices also in $\$ /$ oz. Finally, data on price reductions and other promotions used in the subsequent analysis are also readily available from Dominick's.

Table 3.2 provides NB-related information by manufacturer and segment (Family, Kids, Health, Taste) as in Nevo (2001). These segments cater to different consumer tastes for particular product characteristics. For example, the health brands aim to attract the more health-conscious consumers and have lower sugar content. Overall, the Family and Kids segments are larger, both in terms of brands and sales (dollars and oz.), and can be viewed as mass-market (Mass), while the Health and Taste segments can be viewed as niche (Niche).

Kellogg's and General Mills dominate NBs both in terms of the number of brands and dollar sales. The two manufacturers account for 19 out the top 20 NBs and for more than $95 \%$ of dollar sales. Depending on the segment, prices lie somewhere between 13 cents/oz. for the Health segment of Post and 24 cents/oz. for the Health segment of Kellogg's with a typical box size being 20 oz. The Niche brands are sold at a premium of 4 (2) cents/oz. in the case of General Mills (Kellogg's). Dominick's retail NB margins are between 15\% (General Mills) and 21\% (Post).

Table 3.3 provides similar information for the PLs. As I already mentioned above, Dominick's launched 18 non-imitating PLs in addition to the 6 imitating PLs during this period. These $24 \mathrm{PLs}$ accounted for about $\$ 9$ million dollars in sales with an average price 11 cents/oz - the non-imitating brands account for about $75 \%$ of the 
PL dollar sales. Overall, the PLs enjoy margins of about 30\%, which are notably higher than those for the NBs, with the imitating PLs selling at a higher price and earning a higher dollar margin compared to the non-imitating PLs.

\subsubsection{Demand Estimation}

The demand estimates reported in this section are based on quarterly brand-level sales, prices, and promotions in each of Dominick's four price zones. Given Dominick's employs common pricing and promotion strategies across stores in the same pricing zone on the basis of similarity in consumer demographics and responsiveness to marketing mix, the items at a store in the high price zone are sold at higher prices compared to the medium and low prices zones. Moreover, Domininck's stores in the "Cub-fighter" zone compete head-to-head with Cub Foods stores. There are 20 NBs and 24 PLs and a market is defined as a price zone and quarter combination for a total of 124 markets. To obtain the share of the outside good, I consider the sales for all products, including those not selected for the purpose of demand estimation, as the total market. The share of the outside good is then computed using 1-(inside sales/total sales), with inside sales denoting the sales of the products used in demand estimation.

Tables 3.4 and 3.5 display the OLS and 2SLS estimates for a variety of specifications including price reductions and other promotions (e.g., coupons and "bonus buy"), and price zone dummies among the explanatory variables. I also control for any market conditions that exhibit variation by time only using either a linear time trend or year-by-quarter dummies. In addition, based on my earlier discussion, I use brand dummies instead of detailed product characteristics. To account for the endogeneity of price and the potential endogeneity of price reductions (when applicable) I use the number of other brands owned by the same firm in the same market, as well 
as the number of the other brands owned by the rival firms. Finally, I use standard errors that are robust to hetersokedasticity for the purpose of inference.

Based on the OLS regressions, the price coefficients are negative and highly significant in all models considered and range from -12 to -10.5 . Price reductions and other promotions enter with a positive coefficient, which is expected, and they are mostly statistically significant. The coefficients of the price zone dummies are also mostly significant. The year-by-quarter dummies do not seem to improve the fit of the model compared to the time trend. The average own-price elasticities implied by the OLS estimates are around -2, depending on the model considered (Table 3.6). Note, however, that there is a large number of observations pointing to inelastic demand, which is problematic.

In the case of the 2SLS regressions, based on the p-value of the the score test by Wooldridge (1995), we strongly reject the null hypothesis that price (and price reductions) are exogenous. We also see a very notable increase (in absolute value) in the price coefficients relative to the OLS case, and, hence, our instruments are successful in addressing the endogeneity concerns. Due to the increase in the price coefficient, we also address the issue of observations with inelastic demands. The average own-price elasticities implied by the 2SLS estimates are now between -12 and -7 depending on the model specification.

\subsubsection{Retailer and Manufacturer Pricing}

I report the OLS estimates from the retailer and manufacturer pricing equations (3.23) and (3.24) for which the dependent variables are the differences between observed and predicted margins in Tables 3.7 to 3.10. Note that the estimates are obtained using observations for NBs only. Among the 2,480 total observations, there are 1,736 observations associated with imitated NBs and 744 observations associ- 
ated with non-imitated NBs. Although the results reported here are based on 2SLS demand estimates from model 4, I note that my conclusions remain qualitatively similar using any of the remaining models given that they produce similar substitution patterns.

Overall, I consider up to 12 models for both equations, which differ in the richness of their specifications. For example, I allow for the interaction of the number of PLs offered by Dominick's with dummies indicating whether the NBs are imitated or non-imitated to allow for the possibility that the retailer deviates differently from optimal pricing in the two groups. I also allow for similar interactions in the case of all promotions, as well as when I distinguish between price reductions and other promotions. The ability of models 1-6 to identify deviations from the predicted prices hinges on the assumption that wholesale costs, which are not observable, exhibit variation only by time. In the case of models $7-12$, the identifying assumption is that wholesale costs exhibit variation not only by time but also by manufacturer.

Starting with the retailing pricing equations (3.23) in Tables 3.7 and 3.8, we see that as the number of PLs offered by the retailer increases, the retailer deviates upward from its predicted prices for NBs. Throughout the models considered, the coefficient on the number of PLs (PLs (\#)), or its interactions with the imitated (PLs $(\#) \times$ Imitated) and non-imitated (PLs (\#) $\times$ Non-Imitated) NB dummy is positive and highly significant. Furthermore, its magnitude does not change dramatically across the various models. Hence, in support of the strategic retail pricing hypothesis, Dominick's exerts its strategic power to support the PL gain market share by making the NBs less attractive to the consumers and more so for non-imitated NBs.

Moving to the wholesale pricing equations (3.24), in Tables 3.9 and 3.10, the coefficient on the number of PLs, or its interactions with the imitated and nonimitated NB dummy is negative and highly significant, and its magnitude remains 
largely the same across specifications. Furthermore, the number of PLs has a similar impact on both imitated and non-imitated NBs. Overall, in support of the bargaining power hypothesis, as the number of PLs offered by Dominick's increases, the cereal manufacturers lower the wholesale prices of NBs below their predicted levels.

\subsubsection{Benefits to the Consumers}

I conclude my empirical analysis using a simple back-of-envelope (BOE) exercise to evaluate the benefits of the PLs to the consumers as in Hausman and Leonard (2002). This BOE exercise entails the calculation of consumer surplus under two scenarios. In the baseline scenario, I calculate the consumer surplus for the set of cereal brands observed in the data. In the counterfactual scenario, I calculate the consumer surplus assuming that PLs are not available.

The idea behind this exercise is as follows. Consumers benefit from PLs in two ways. First, they enjoy the increased variety of cereal brands offered by the retailer (variety effect). Additionally, the presence of PLs creates increased competition for the existing NBs (price effect). Although, the overall effect on the consumers is then the sum of the variety and the price effects, the current version of my exercise ignores the price effect and focuses on the variety effect only. That is, I only remove the PLs from the consumers' choice set without changing the price of the remaining brands.

Using base and $c f$ to refer to the baseline and counterfactual scenarios, respectively, the consumer surplus in each market - defined as a price zone $(z)$ and quarter (t) combination - for scenario $k$ is given by

$$
C S_{z t}^{k}=\frac{\log \left(\sum_{j=1}^{N_{z t}^{k}} \exp \left(\delta_{j z t}\right)\right)}{a}, \quad k \in\{\text { base }, c f\}
$$

where $\delta_{j z t}$ is the mean utility level associated with brand $j$ in zone $z$ and quarter $t$, 
$N_{z t}^{k}$ is the number of brands in the market under consideration for scenario $k$, and $a$ is the price coefficient (marginal utility of income); see, for example, Trajtenberg (1989). Note also that $N_{z t}^{b a s e}>N_{z t}^{c f}$. Recall that Dominick's uses 4 pricing zones with common pricing and marketing mix across the stores in the same zones and the data span the period from September 1989 to May 1997.

In Table 3.11, I provide the values of consumer surplus for both the baseline and the counterfactual scenario by price zone. The High and Medium price zones account for roughly $70 \%$ of the total benefit to the consumers, which is close to $\$ 1.2$ million. Note that these are the price zones with the most units sold.

\subsection{Conclusion}

In this chapter, I test two hypotheses regarding the use of private labels by a retailer. The first is whether private labels enhance the retailer's ability to bargain for lower wholesale price with its national brand manufacturers; the so-called bargaining power hypothesis. The second is whether the retailer strategically sets retail prices for all brands to favor its private labels - the strategic retailer pricing hypothesis. I test the two hypotheses using cereal data from a large super-market chain in Chicago between 1989 and 1997. My empirical strategy for testing both hypotheses controls for the effect of the changed market structure on wholesale and retail prices due to the presence of private labels using a structural approach.

In support of the bargaining power hypothesis, I find that a larger number of private labels allows the retailer to bargain for wholesale prices with its national brand manufacturers below their predicted levels. In support of the strategic retailer pricing hypothesis, a larger number of private labels also leads to an increase in retail prices for the national brands above their predicted levels.

In addition, a counterfactual scenario is simulated to study the consumer surplus 
in the absence of private labels. The simulation results show that consumer surplus would be reduced if private labels were not introduced, suggesting that consumers benefited from the presence of private labels. 
Table 3.1: National brands

\begin{tabular}{lllllll}
\hline \multicolumn{1}{c}{ Description } & \multicolumn{1}{c}{ Manuf. } & Segment & Dollars & Oz & Price $(\$ /$ oz $)$ & Margin $(\$ /$ oz $)$ \\
\hline CHEERIOS* & General Mills & Family & $\$ 11,677,181$ & $58,873,160$ & $\$ 0.20$ & $\$ 0.02$ \\
FROSTED FLAKES* & Kellogg's & Family & $\$ 11,269,938$ & $71,938,082$ & $\$ 0.16$ & $\$ 0.02$ \\
RICE KRISPIES* & Kellogg's & Family & $\$ 9,717,795$ & $54,213,950$ & $\$ 0.18$ & $\$ 0.02$ \\
HONEY NUT CHEERIOS & General Mills & Family & $\$ 8,348,791$ & $43,909,154$ & $\$ 0.19$ & $\$ 0.02$ \\
CORN FLAKES* & Kellogg's & Family & $\$ 7,901,504$ & $70,703,033$ & $\$ 0.11$ & $\$ 0.02$ \\
RAISIN BRAN* & Kellogg's & Taste & $\$ 7,225,190$ & $49,553,193$ & $\$ 0.15$ & $\$ 0.02$ \\
SPECIAL K & Kellogg's & Health & $\$ 6,008,240$ & $26,491,374$ & $\$ 0.23$ & $\$ 0.03$ \\
LUCKY CHARMS & General Mills & Kids & $\$ 4,768,835$ & $22,036,410$ & $\$ 0.22$ & $\$ 0.03$ \\
CINNAMON TOAST CRUNCH & General Mills & Kids & $\$ 4,184,018$ & $19,610,423$ & $\$ 0.21$ & $\$ 0.03$ \\
GOLDEN GRAHAMS & General Mills & Family & $\$ 4,148,933$ & $19,797,138$ & $\$ 0.21$ & $\$ 0.03$ \\
CORN POPS & Kellogg's & Kids & $\$ 3,975,982$ & $19,286,525$ & $\$ 0.21$ & $\$ 0.02$ \\
FRUIT LOOPS* & Kellogg's & Kids & $\$ 3,941,180$ & $20,113,867$ & $\$ 0.20$ & $\$ 0.03$ \\
WHEATIES & General Mills & Family & $\$ 3,664,419$ & $21,475,122$ & $\$ 0.17$ & $\$ 0.02$ \\
TRIX & General Mills & Kids & $\$ 3,618,624$ & $14,954,011$ & $\$ 0.24$ & $\$ 0.03$ \\
CRISPIX & Kellogg's & Family & $\$ 1,149,713$ & $5,502,647$ & $\$ 0.21$ & $\$ 0.04$ \\
APPLE JACKS & Kellogg's & Kids & $\$ 3,339,607$ & $15,257,595$ & $\$ 0.22$ & $\$ 0.03$ \\
WHOLE GRAIN TOTAL & General Mills & Health & $\$ 2,983,540$ & $12,221,496$ & $\$ 0.24$ & $\$ 0.04$ \\
KIX & General Mills & Kids & $\$ 2,926,832$ & $11,691,281$ & $\$ 0.25$ & $\$ 0.04$ \\
CRACKLIN OAT BRAN & Kellogg's & Taste & $\$ 2,818,125$ & $13,120,335$ & $\$ 0.21$ & $\$ 0.03$ \\
GRAPE-NUTS CERE & Post & Health & $\$ 2,756,625$ & $21,120,288$ & $\$ 0.13$ & $\$ 0.02$ \\
\hline \multicolumn{1}{c}{ Total } & & & $\$ 106,425,070$ & $591,869,084$ & $\$ 0.18$ & $\$ 0.02$ \\
\hline
\end{tabular}

Note: National brands imitated by Dominick's PLs are indicated by an asterisk. 
Table 3.2: National brands by manufacturer/segment

\begin{tabular}{|c|c|c|c|c|c|c|c|}
\hline Manufacturer & Family & Kids & Health & Taste & Mass & Niche & Total \\
\hline General Mills & 4 & 4 & 1 & & 8 & 1 & 9 \\
\hline Kellogg's & 4 & 3 & 1 & 2 & 7 & 3 & 10 \\
\hline Post & & & 1 & & & 1 & 1 \\
\hline Total & 8 & 7 & 3 & 2 & 15 & 5 & 20 \\
\hline \multicolumn{8}{|c|}{ (a) brands } \\
\hline Manufacturer & Family & Kids & Health & Taste & Mass & Niche & Total \\
\hline General Mills & $\$ 27,839,323$ & $\$ 15,498,309$ & $\$ 2,983,540$ & & $\$ 43,337,632$ & $\$ 2,983,540$ & $\$ 46,321,172$ \\
\hline Kellogg's & $\$ 30,038,950$ & $\$ 11,256,769$ & $\$ 6,008,240$ & $\$ 10,043,314$ & $\$ 41,295,719$ & $\$ 16,051,554$ & $\$ 57,347,273$ \\
\hline Post & & & $\$ 2,756,625$ & & & $\$ 2,756,625$ & $\$ 2,756,625$ \\
\hline Total & $\$ 57,878,273$ & $\$ 26,755,077$ & $\$ 11,748,405$ & $\$ 10,043,314$ & $\$ 84,633,351$ & $\$ 21,791,719$ & $\$ 106,425,070$ \\
\hline
\end{tabular}
(b) dollars, retail

\begin{tabular}{lccccccc}
\hline Manufacturer & Family & Kids & Health & Taste & Mass & Niche & Total \\
\hline General Mills & $\$ 0.19$ & $\$ 0.23$ & $\$ 0.24$ & & $\$ 0.20$ & $\$ 0.24$ & $\$ 0.21$ \\
Kellogg's & $\$ 0.15$ & $\$ 0.21$ & $\$ 0.23$ & $\$ 0.16$ & $\$ 0.16$ & $\$ 0.18$ & $\$ 0.13$ \\
Post & & $\$ 0.13$ & & $\$ 0.13$ \\
\hline \multicolumn{1}{c}{ Total } & $\$ 0.17$ & $\$ 0.22$ & $\$ 0.20$ & $\$ 0.16$ & $\$ 0.18$ & $\$ 0.18$ \\
\hline
\end{tabular}

(c) price (\$/oz.), retail

\begin{tabular}{lccccccc}
\hline Manufacturer & Family & Kids & Health & Taste & Mass & Niche & Total \\
\hline General Mills & $\$ 0.17$ & $\$ 0.20$ & $\$ 0.21$ & & $\$ 0.18$ & $\$ 0.21$ & $\$ 0.18$ \\
Kellogg's & $\$ 0.13$ & $\$ 0.18$ & $\$ 0.19$ & $\$ 0.14$ & $\$ 0.14$ & $\$ 0.16$ & $\$ 0.14$ \\
Post & & & $\$ 0.11$ & & & $\$ 0.11$ & $\$ 0.11$ \\
\hline \multicolumn{1}{c}{ Total } & $\$ 0.15$ & $\$ 0.19$ & $\$ 0.17$ & $\$ 0.14$ & $\$ 0.16$ & $\$ 0.15$ & $\$ 0.16$ \\
\hline
\end{tabular}

(d) price (\$/oz.), wholesale

Note: The Family and Kids segment can be viewed as "mass-market" while the Health and Taste segments can be viewed as "niche".

Table 3.3: Private labels

\begin{tabular}{lccccc}
\hline & Brands & Dollars & Oz & Price $(\$ /$ oz $)$ & Margin $(\$ /$ oz $)$ \\
\hline Non-Imitating & 18 & $\$ 6,919,777.47$ & $65,402,587$ & $\$ 0.11$ & $\$ 0.03$ \\
Imitating & 6 & $\$ 2,102,634.87$ & $17,014,315$ & $\$ 0.12$ & $\$ 0.04$ \\
\hline Total & 24 & $\$ 9,022,412.34$ & $82,416,902$ & $\$ 0.11$ & $\$ 0.03$ \\
\hline
\end{tabular}


Table 3.4: Logit demand estimates: OLS

\begin{tabular}{|c|c|c|c|c|c|c|}
\hline VARIABLES & $(1)$ & $(2)$ & (3) & $(4)$ & $(5)$ & $(6)$ \\
\hline Price & $\begin{array}{c}-11.8888 * * * \\
(0.4006)\end{array}$ & $\begin{array}{c}-10.5034 * * * \\
(0.4511)\end{array}$ & $\begin{array}{c}-10.5770 * * * \\
(0.4772)\end{array}$ & $\begin{array}{c}-12.0205 * * * \\
(0.3993)\end{array}$ & $\begin{array}{c}-10.6416^{* * *} \\
(0.4492)\end{array}$ & $\begin{array}{c}-10.7987 * * * \\
(0.4761)\end{array}$ \\
\hline Price Reduction & $\begin{array}{l}0.0004 * \\
(0.0002)\end{array}$ & $\begin{array}{c}0.0017 * * * \\
(0.0003)\end{array}$ & $\begin{array}{c}0.0030 * * * \\
(0.0004)\end{array}$ & $\begin{array}{c}0.0001 \\
(0.0002)\end{array}$ & $\begin{array}{c}0.0014 * * * \\
(0.0003)\end{array}$ & $\begin{array}{c}0.0025 * * * \\
(0.0003)\end{array}$ \\
\hline Other Promotions & $\begin{array}{c}0.0008 * * * \\
(0.0001)\end{array}$ & $\begin{array}{c}0.0008 * * * \\
(0.0001)\end{array}$ & $\begin{array}{c}0.0011 * * * \\
(0.0001)\end{array}$ & & & \\
\hline Time Trend & & $\begin{array}{c}-0.0074 * * * \\
(0.0008)\end{array}$ & & & $\begin{array}{c}-0.0074 * * * \\
(0.0008)\end{array}$ & \\
\hline Zone 2 & $\begin{array}{c}-0.1150 * * * \\
(0.0198)\end{array}$ & $\begin{array}{c}-0.1147 * * * \\
(0.0197)\end{array}$ & $\begin{array}{c}-0.1244 * * * \\
(0.0191)\end{array}$ & $\begin{array}{c}-0.1033 * * * \\
(0.0197)\end{array}$ & $\begin{array}{c}-0.1028^{* * *} \\
(0.0196)\end{array}$ & $\begin{array}{c}-0.1085^{* * *} \\
(0.0189)\end{array}$ \\
\hline Zone 3 & $\begin{array}{c}0.0302 \\
(0.0205)\end{array}$ & $\begin{array}{c}0.0472 * * \\
(0.0204)\end{array}$ & $\begin{array}{c}0.0614 * * * \\
(0.0202)\end{array}$ & $\begin{array}{c}0.0108 \\
(0.0198)\end{array}$ & $\begin{array}{c}0.0276 \\
(0.0196)\end{array}$ & $\begin{array}{c}0.0344 * \\
(0.0193)\end{array}$ \\
\hline Zone 4 & $\begin{array}{c}-0.1039 * * * \\
(0.0205)\end{array}$ & $\begin{array}{c}-0.0808 * * * \\
(0.0205)\end{array}$ & $\begin{array}{c}-0.0662 * * * \\
(0.0204)\end{array}$ & $\begin{array}{c}-0.1244 * * * \\
(0.0196)\end{array}$ & $\begin{array}{c}-0.1016 * * * \\
(0.0197)\end{array}$ & $\begin{array}{c}-0.0949 * * * \\
(0.0193)\end{array}$ \\
\hline Constant & $\begin{array}{c}-0.0544 \\
(0.0863) \\
\end{array}$ & $\begin{array}{c}-0.2455^{* * *} \\
(0.0909)\end{array}$ & $\begin{array}{c}-0.3233 * * * \\
(0.1046) \\
\end{array}$ & $\begin{array}{c}0.0101 \\
(0.0850) \\
\end{array}$ & $\begin{array}{c}-0.1795 * * \\
(0.0896) \\
\end{array}$ & $\begin{array}{c}-0.2547 * * \\
(0.1039) \\
\end{array}$ \\
\hline Brand Dummies & $\mathrm{X}$ & $\mathrm{X}$ & $\mathrm{X}$ & $\mathrm{X}$ & $\mathrm{X}$ & X \\
\hline Time Trend & & $\mathrm{X}$ & & & $\mathrm{X}$ & \\
\hline Year x Quarter Dummies & & & $\mathrm{X}$ & & & $\mathrm{X}$ \\
\hline Obs. & 3945 & 3945 & 3945 & 3945 & 3945 & 3945 \\
\hline R-squared & 0.9159 & 0.9173 & 0.9231 & 0.9155 & 0.9168 & 0.9225 \\
\hline
\end{tabular}

Note: Robust standard errors in parentheses. Brand dummies are included but not displayed. The asterisks denote statistical significance as follows: *** $(1 \%),{ }^{* *}(5 \%),{ }^{*}(10 \%)$. 
Table 3.5: Logit demand estimates: IV

\begin{tabular}{|c|c|c|c|c|c|c|}
\hline VARIABLES & $(1)$ & $(2)$ & (3) & $(4)$ & $(5)$ & $(6)$ \\
\hline Price & $\begin{array}{c}-43.3153 * * * \\
(5.2025)\end{array}$ & $\begin{array}{c}-68.6720 * * * \\
(11.5446)\end{array}$ & $\begin{array}{c}-59.9376 * * * \\
(18.8486)\end{array}$ & $\begin{array}{c}-42.8987 * * * \\
(5.0421)\end{array}$ & $\begin{array}{c}-68.2083 * * * \\
(11.6590)\end{array}$ & $\begin{array}{c}-66.9903 * * \\
(28.7551)\end{array}$ \\
\hline Price Reduction & $\begin{array}{c}0.0209 * * * \\
(0.0054)\end{array}$ & $\begin{array}{c}0.0011 \\
(0.0057)\end{array}$ & $\begin{array}{l}-0.0189 \\
(0.0165)\end{array}$ & $\begin{array}{c}0.0204 * * * \\
(0.0052)\end{array}$ & $\begin{array}{c}0.0013 \\
(0.0064)\end{array}$ & $\begin{array}{l}-0.0232 \\
(0.0234)\end{array}$ \\
\hline Other Promotions & $\begin{array}{c}0.0021 * * * \\
(0.0005)\end{array}$ & $\begin{array}{c}0.0002 \\
(0.0006)\end{array}$ & $\begin{array}{l}-0.0019 \\
(0.0020)\end{array}$ & & & \\
\hline Time Trend & & $\begin{array}{c}0.0404^{* * *} \\
(0.0100)\end{array}$ & & & $\begin{array}{c}0.0398 * * * \\
(0.0111)\end{array}$ & \\
\hline Zone 2 & $\begin{array}{c}-0.4056 * * * \\
(0.0736)\end{array}$ & $\begin{array}{c}-0.4120 * * * \\
(0.0927)\end{array}$ & $\begin{array}{c}-0.2356 * * * \\
(0.0618)\end{array}$ & $\begin{array}{c}-0.3731 * * * \\
(0.0675)\end{array}$ & $\begin{array}{c}-0.4086^{* * *} \\
(0.0887)\end{array}$ & $\begin{array}{c}-0.2757 * * * \\
(0.0696)\end{array}$ \\
\hline Zone 3 & $\begin{array}{c}0.0402 \\
(0.0429)\end{array}$ & $\begin{array}{c}-0.2822 * * * \\
(0.0893)\end{array}$ & $\begin{array}{l}-0.4278^{*} \\
(0.2561)\end{array}$ & $\begin{array}{l}-0.0031 \\
(0.0388)\end{array}$ & $\begin{array}{c}-0.2813 * * * \\
(0.0906)\end{array}$ & $\begin{array}{l}-0.4603 \\
(0.3238)\end{array}$ \\
\hline Zone 4 & $\begin{array}{c}-0.2220 * * * \\
(0.0409)\end{array}$ & $\begin{array}{c}-0.6556 * * * \\
(0.1249)\end{array}$ & $\begin{array}{c}-0.7681 * * \\
(0.3355)\end{array}$ & $\begin{array}{c}-0.2649 * * * \\
(0.0403)\end{array}$ & $\begin{array}{c}-0.6527 * * * \\
(0.1285)\end{array}$ & $\begin{array}{l}-0.8295^{*} \\
(0.4452)\end{array}$ \\
\hline Constant & $\begin{array}{c}5.8758 * * * \\
(0.9572)\end{array}$ & $\begin{array}{c}10.8949 * * * \\
(2.2010)\end{array}$ & $\begin{array}{l}9.1027 * * \\
(3.6937)\end{array}$ & $\begin{array}{c}5.8812 * * * \\
(0.9417)\end{array}$ & $\begin{array}{c}10.8121 * * * \\
(2.2267)\end{array}$ & $\begin{array}{l}10.4163 * \\
(5.5685)\end{array}$ \\
\hline Brand Dummies & $\mathrm{X}$ & $\mathrm{X}$ & $\mathrm{X}$ & $\mathrm{X}$ & $\mathrm{X}$ & $\mathrm{X}$ \\
\hline Time Trend & & $\mathrm{X}$ & & & $\mathrm{X}$ & \\
\hline Year x Quarter Dummies & & & $\mathrm{X}$ & & & $\mathrm{X}$ \\
\hline Obs. & 3945 & 3945 & 3945 & 3945 & 3945 & 3945 \\
\hline Exog. test & $\begin{array}{l}168.00 \\
{[0.000]}\end{array}$ & $\begin{array}{c}118.70 \\
{[0.000]}\end{array}$ & $\begin{array}{c}76.68 \\
{[0.000]}\end{array}$ & $\begin{array}{l}160.70 \\
{[0.000]}\end{array}$ & $\begin{array}{l}110.50 \\
{[0.000]}\end{array}$ & $\begin{array}{c}72.38 \\
{[0.000]}\end{array}$ \\
\hline
\end{tabular}

Note: Robust standard errors in parentheses. Brand dummies are included but not displayed. The asterisks denote statistical significance as follows: *** $(1 \%), * *(5 \%), *(10 \%)$. P-values for the exogeneity test of Wooldridge (1995) in squared brackets.

Table 3.6: Own price elasticities: summary statistics by model

\begin{tabular}{ccccc}
\hline & \multicolumn{2}{c}{ OLS } & \multicolumn{2}{c}{ 2SLS } \\
\cline { 2 - 5 } model & mean & std. deviation & mean & std. deviation \\
\hline 3 & -1.81 & 0.54 & -10.28 & 3.03 \\
4 & -2.06 & 0.61 & -7.36 & 2.17 \\
5 & -1.82 & 0.54 & -11.70 & 3.45 \\
6 & -1.85 & 0.55 & -11.49 & 3.39 \\
\hline
\end{tabular}

Note: The summary statistics reported in this table are based on the OLS and 2SLS demand estimates for models 3, 4, 5, and 6 reported in Tables 3.4 and 3.5. 
Table 3.7: Retailer Pricing Model I

\begin{tabular}{|c|c|c|c|c|c|c|}
\hline VARIABLES & (1) & (2) & (3) & (4) & (5) & (6) \\
\hline PLs (\#) & $\begin{array}{c}0.0029^{* * *} \\
(0.0005)\end{array}$ & $\begin{array}{c}0.0033 * * * \\
(0.0006)\end{array}$ & & & & \\
\hline PLs $(\#) \times$ Imitated & & & $\begin{array}{c}0.0023 * * * \\
(0.0005)\end{array}$ & $\begin{array}{c}0.0028 * * * \\
(0.0006)\end{array}$ & $\begin{array}{c}0.0027 * * * \\
(0.0006)\end{array}$ & $\begin{array}{c}0.0026^{* * *} \\
(0.0006)\end{array}$ \\
\hline PLs $(\#) \times$ Non-Imitated & & & $\begin{array}{c}0.0031^{* * *} \\
(0.0005)\end{array}$ & $\begin{array}{c}0.0035^{* * *} \\
(0.0006)\end{array}$ & $\begin{array}{c}0.0036^{* * *} \\
(0.0006)\end{array}$ & $\begin{array}{c}0.0035^{* * *} \\
(0.0006)\end{array}$ \\
\hline Imitated & $\begin{array}{c}-0.0098^{* * *} \\
(0.0006)\end{array}$ & $\begin{array}{c}-0.0097 * * * \\
(0.0007)\end{array}$ & $\begin{array}{l}-0.0001 \\
(0.0013)\end{array}$ & $\begin{array}{l}-0.0007 \\
(0.0013)\end{array}$ & $\begin{array}{l}-0.0007 \\
(0.0013)\end{array}$ & $\begin{array}{l}-0.0004 \\
(0.0013)\end{array}$ \\
\hline All Promotions $\times$ Imitated & & $\begin{array}{l}-0.0000 * \\
(0.0000)\end{array}$ & & & & $\begin{array}{l}-0.0000 \\
(0.0000)\end{array}$ \\
\hline All Promotions $\times$ Non-Imitated & & $\begin{array}{c}-0.0000^{* * *} \\
(0.0000)\end{array}$ & & & & $\begin{array}{c}-0.0000^{* * *} \\
(0.0000)\end{array}$ \\
\hline Price Reductions & & & & $\begin{array}{c}-0.0000^{* * *} \\
(0.0000)\end{array}$ & & \\
\hline Other Promotions & & & & $\begin{array}{l}-0.0000 \\
(0.0000)\end{array}$ & & \\
\hline Price Reductions $\times$ Imitated & & & & & $\begin{array}{l}-0.0000 \\
(0.0000)\end{array}$ & \\
\hline Price Reductions $\times$ Non-Imitated & & & & & $\begin{array}{c}-0.0001 * * * \\
(0.0000)\end{array}$ & \\
\hline Other Promotions $\times$ Imitated & & & & & $\begin{array}{c}0.0000 \\
(0.0000)\end{array}$ & \\
\hline Other Promotions $\times$ Non-Imitated & & & & & $\begin{array}{l}-0.0000 \\
(0.0000)\end{array}$ & \\
\hline Constant & $\begin{array}{c}-0.0217 * * * \\
(0.0018) \\
\end{array}$ & $\begin{array}{c}-0.0225^{* * *} \\
(0.0018) \\
\end{array}$ & $\begin{array}{c}-0.0246^{* * *} \\
(0.0018) \\
\end{array}$ & $\begin{array}{c}-0.0252^{* * *} \\
(0.0018) \\
\end{array}$ & $\begin{array}{c}-0.0252^{* * *} \\
(0.0018) \\
\end{array}$ & $\begin{array}{c}-0.0252 * * * \\
(0.0018)\end{array}$ \\
\hline Obs. & 2,480 & 2,480 & 2,480 & 2,480 & 2,480 & 2,480 \\
\hline R-squared & 0.4730 & 0.4751 & 0.4832 & 0.4861 & 0.4873 & 0.4856 \\
\hline
\end{tabular}

Note: Robust standard errors in parentheses. Quarter-by-year dummies are included but not repefted. The asterisks denote statistical significance as follows: *** $(1 \%), * *(5 \%), *(10 \%)$.. 
Table 3.8: Retailer Pricing Model II

\begin{tabular}{|c|c|c|c|c|c|c|}
\hline VARIABLES & $(7)$ & $(8)$ & $(9)$ & $(10)$ & $(11)$ & $(12)$ \\
\hline \multirow[t]{2}{*}{ PLs $(\#) \times$ Imitated } & $0.0027 * * *$ & $0.0026^{* * *}$ & $0.0026^{* * *}$ & $0.0026^{* * *}$ & $0.0027 * * *$ & $0.0026^{* * *}$ \\
\hline & $(0.0006)$ & $(0.0006)$ & $(0.0006)$ & $(0.0006)$ & $(0.0006)$ & $(0.0006)$ \\
\hline \multirow[t]{2}{*}{ PLs $(\#) \times$ Non-Imitated } & $0.0036^{* * *}$ & $0.0035 * * *$ & $0.0036^{* * *}$ & $0.0035 * * *$ & $0.0036 * * *$ & $0.0035 * * *$ \\
\hline & $(0.0006)$ & $(0.0005)$ & $(0.0005)$ & $(0.0005)$ & $(0.0006)$ & $(0.0005)$ \\
\hline \multirow[t]{2}{*}{ Imitated } & -0.0017 & -0.0014 & & & & \\
\hline & $(0.0014)$ & $(0.0013)$ & & & & \\
\hline \multirow[t]{2}{*}{ All Promotions $\times$ Imitated } & & -0.0000 & & -0.0000 & & -0.0000 \\
\hline & & $(0.0000)$ & & $(0.0000)$ & & $(0.0000)$ \\
\hline \multirow[t]{2}{*}{ All Promotions $\times$ Non-Imitated } & & $-0.0000 * * *$ & & $-0.0000 * * *$ & & $-0.0000 * * *$ \\
\hline & & $(0.0000)$ & & $(0.0000)$ & & $(0.0000)$ \\
\hline \multirow[t]{2}{*}{ Price Reductions $\times$ Imitated } & -0.0000 & & -0.0000 & & -0.0000 & \\
\hline & $(0.0000)$ & & $(0.0000)$ & & $(0.0000)$ & \\
\hline \multirow[t]{2}{*}{ Price Reductions $\times$ Non-Imitated } & $-0.0001 * * *$ & & $-0.0001 * * *$ & & $-0.0001 * * *$ & \\
\hline & $(0.0000)$ & & $(0.0000)$ & & $(0.0000)$ & \\
\hline \multirow[t]{2}{*}{ Other Promotions $\times$ Imitated } & 0.0000 & & 0.0000 & & 0.0000 & \\
\hline & $(0.0000)$ & & $(0.0000)$ & & $(0.0000)$ & \\
\hline \multirow[t]{2}{*}{ Other Promotions $\times$ Non-Imitated } & -0.0000 & & -0.0000 & & -0.0000 & \\
\hline & $(0.0000)$ & & $(0.0000)$ & & $(0.0000)$ & \\
\hline \multirow[t]{2}{*}{ General Mills } & $0.0106^{* * *}$ & $0.0105 * * *$ & $0.0106 * * *$ & $0.0105 * * *$ & $0.0102 * * *$ & $0.0102 * * *$ \\
\hline & $(0.0008)$ & $(0.0008)$ & $(0.0008)$ & $(0.0008)$ & $(0.0008)$ & $(0.0008)$ \\
\hline \multirow[t]{2}{*}{ Kellogg's } & $0.0112 * * *$ & $0.0111 * * *$ & $0.0110^{* * *}$ & $0.0110^{* * *}$ & $0.0117 * * *$ & $0.0117 * * *$ \\
\hline & $(0.0009)$ & $(0.0009)$ & $(0.0009)$ & $(0.0009)$ & $(0.0009)$ & $(0.0009)$ \\
\hline \multirow[t]{2}{*}{ General Mills $\times$ Imitated } & & & & & 0.0013 & 0.0017 \\
\hline & & & & & $(0.0016)$ & $(0.0015)$ \\
\hline \multirow[t]{2}{*}{ Kellogg's $\times$ Imitated } & & & & & $-0.0028 * *$ & $-0.0025^{*}$ \\
\hline & & & & & $(0.0014)$ & $(0.0013)$ \\
\hline \multirow[t]{2}{*}{ Constant } & $-0.0353 * * *$ & $-0.0351 * * *$ & $-0.0357 * * *$ & $-0.0355 * * *$ & $-0.0353 * * *$ & $-0.0351 * * *$ \\
\hline & $(0.0019)$ & $(0.0019)$ & $(0.0019)$ & $(0.0019)$ & $(0.0019)$ & $(0.0019)$ \\
\hline Obs. & 2,480 & 2,480 & 2,480 & 2,480 & 2,480 & 2,480 \\
\hline R-squared & 0.5058 & 0.5040 & 0.5056 & 0.5038 & 0.5077 & 0.5059 \\
\hline
\end{tabular}

Note: Robust standard errors in parentheses. Quarter-by-year dummies are included but not rePrefted. The asterisks denote statistical significance as follows: *** $(1 \%), * *(5 \%), *(10 \%)$. 
Table 3.9: Manufacturer Pricing Model I

\begin{tabular}{|c|c|c|c|c|c|c|}
\hline VARIABLES & (1) & (2) & (3) & (4) & $(5)$ & (6) \\
\hline PLs (\#) & $\begin{array}{c}-0.0067^{* * *} \\
(0.0014)\end{array}$ & $\begin{array}{c}-0.0046^{* * *} \\
(0.0014)\end{array}$ & & & & \\
\hline PLs $(\#) \times$ Imitated & & & $\begin{array}{c}-0.0067^{* * *} \\
(0.0014)\end{array}$ & $\begin{array}{c}-0.0041 * * * \\
(0.0014)\end{array}$ & $\begin{array}{c}-0.0043 * * * \\
(0.0014)\end{array}$ & $\begin{array}{c}-0.0046^{* * *} \\
(0.0014)\end{array}$ \\
\hline PLs $(\#) \times$ Non-Imitated & & & $\begin{array}{c}-0.0067^{* * *} \\
(0.0014)\end{array}$ & $\begin{array}{c}-0.0046^{* * *} \\
(0.0014)\end{array}$ & $\begin{array}{c}-0.0043 * * * \\
(0.0014)\end{array}$ & $\begin{array}{c}-0.0046^{* * *} \\
(0.0014)\end{array}$ \\
\hline Imitated & $\begin{array}{c}-0.0381^{* * *} \\
(0.0014)\end{array}$ & $\begin{array}{c}-0.0390 * * * \\
(0.0019)\end{array}$ & $\begin{array}{c}-0.0385 * * * \\
(0.0038)\end{array}$ & $\begin{array}{c}-0.0406^{* * *} \\
(0.0038)\end{array}$ & $\begin{array}{c}-0.0385 * * * \\
(0.0040)\end{array}$ & $\begin{array}{c}-0.0393 * * * \\
(0.0038)\end{array}$ \\
\hline All Promotions $\times$ Imitated & & $\begin{array}{c}-0.0001^{* *} \\
(0.0000)\end{array}$ & & & & $\begin{array}{c}-0.0001 * * \\
(0.0000)\end{array}$ \\
\hline All Promotions $\times$ Non-Imitated & & $\begin{array}{c}-0.0002 * * * \\
(0.0000)\end{array}$ & & & & $\begin{array}{c}-0.0002 * * * \\
(0.0000)\end{array}$ \\
\hline Price Reductions & & & & $\begin{array}{c}-0.0002 * * * \\
(0.0000)\end{array}$ & & \\
\hline Other Promotions & & & & $\begin{array}{c}-0.0001^{* * *} \\
(0.0000)\end{array}$ & & \\
\hline Price Reductions $\times$ Imitated & & & & & $\begin{array}{c}-0.0001^{* * *} \\
(0.0000)\end{array}$ & \\
\hline Price Reductions $\times$ Non-Imitated & & & & & $\begin{array}{c}-0.0003 * * * \\
(0.0001)\end{array}$ & \\
\hline Other Promotions $\times$ Imitated & & & & & $\begin{array}{l}-0.0000 \\
(0.0000)\end{array}$ & \\
\hline Other Promotions $\times$ Non-Imitated & & & & & $\begin{array}{c}-0.0001 * * * \\
(0.0000)\end{array}$ & \\
\hline Constant & $\begin{array}{c}0.0940 * * * \\
(0.0042)\end{array}$ & $\begin{array}{c}0.0904 * * * \\
(0.0042)\end{array}$ & $\begin{array}{c}0.0941 * * * \\
(0.0043)\end{array}$ & $\begin{array}{c}0.0905 * * * \\
(0.0043)\end{array}$ & $\begin{array}{c}0.0895 * * * \\
(0.0043)\end{array}$ & $\begin{array}{c}0.0905 * * * \\
(0.0043)\end{array}$ \\
\hline Obs. & 2,480 & 2,480 & 2,480 & 2,480 & 2,480 & 2,480 \\
\hline R-squared & 0.3465 & 0.3580 & 0.3465 & 0.3595 & 0.3631 & 0.3580 \\
\hline
\end{tabular}

Note: Robust standard errors in parentheses. Quarter-by-year dummies are included but not reportêd. The asterisks denote statistical significance as follows: *** (1\%), **(5\%), *(10\%). 
Table 3.10: Manufacturer Pricing Model II

\begin{tabular}{|c|c|c|c|c|c|c|}
\hline VARIABLES & (7) & (8) & (9) & (10) & (11) & (12) \\
\hline PLs $(\#) \times$ Imitated & $\begin{array}{c}-0.0042 * * * \\
(0.0012)\end{array}$ & $\begin{array}{c}-0.0045^{* * *} \\
(0.0012)\end{array}$ & $\begin{array}{c}-0.0063^{* * *} \\
(0.0012)\end{array}$ & $\begin{array}{c}-0.0065^{* * *} \\
(0.0012)\end{array}$ & $\begin{array}{c}-0.0042^{* * *} \\
(0.0012)\end{array}$ & $\begin{array}{c}-0.0045^{* * *} \\
(0.0012)\end{array}$ \\
\hline PLs $(\#) \times$ Non-Imitated & $\begin{array}{c}-0.0041^{* * *} \\
(0.0012)\end{array}$ & $\begin{array}{c}-0.0045^{* * *} \\
(0.0012)\end{array}$ & $\begin{array}{c}-0.0032 * * * \\
(0.0012)\end{array}$ & $\begin{array}{c}-0.0036^{* * *} \\
(0.0012)\end{array}$ & $\begin{array}{c}-0.0042^{* * *} \\
(0.0012)\end{array}$ & $\begin{array}{c}-0.0046^{* * *} \\
(0.0012)\end{array}$ \\
\hline Imitated & $\begin{array}{c}-0.0412^{* * *} \\
(0.0036)\end{array}$ & $\begin{array}{c}-0.0422 * * * \\
(0.0035)\end{array}$ & & & & \\
\hline All Promotions $\times$ Imitated & & $\begin{array}{c}-0.0001^{* *} \\
(0.0000)\end{array}$ & & $\begin{array}{c}-0.0001 * * * \\
(0.0000)\end{array}$ & & $\begin{array}{c}-0.0001^{* * *} \\
(0.0000)\end{array}$ \\
\hline All Promotions $\times$ Non-Imitated & & $\begin{array}{c}-0.0002 * * * \\
(0.0000)\end{array}$ & & $\begin{array}{c}-0.0002 * * * \\
(0.0000)\end{array}$ & & $\begin{array}{c}-0.0002 * * * \\
(0.0000)\end{array}$ \\
\hline Price Reductions $\times$ Imitated & $\begin{array}{c}-0.0001^{* * *} \\
(0.0000)\end{array}$ & & $\begin{array}{l}-0.0000 \\
(0.0000)\end{array}$ & & $\begin{array}{c}-0.0001 * * * \\
(0.0000)\end{array}$ & \\
\hline Price Reductions $\times$ Non-Imitated & $\begin{array}{c}-0.0004^{* * *} \\
(0.0000)\end{array}$ & & $\begin{array}{c}-0.0004^{* * *} \\
(0.0000)\end{array}$ & & $\begin{array}{c}-0.0004^{* * *} \\
(0.0000)\end{array}$ & \\
\hline Other Promotions $\times$ Imitated & $\begin{array}{l}-0.0000 \\
(0.0000)\end{array}$ & & $\begin{array}{c}-0.0001^{* * *} \\
(0.0000)\end{array}$ & & $\begin{array}{l}-0.0000^{*} \\
(0.0000)\end{array}$ & \\
\hline Other Promotions $\times$ Non-Imitated & $\begin{array}{c}-0.0001 * * * \\
(0.0000)\end{array}$ & & $\begin{array}{c}-0.0001 * * * \\
(0.0000)\end{array}$ & & $\begin{array}{c}-0.0001 * * * \\
(0.0000)\end{array}$ & \\
\hline General Mills & $\begin{array}{c}0.0839 * * * \\
(0.0016)\end{array}$ & $\begin{array}{c}0.0836 * * * \\
(0.0016)\end{array}$ & $\begin{array}{c}0.0833^{* * *} \\
(0.0017)\end{array}$ & $\begin{array}{c}0.0829 * * * \\
(0.0018)\end{array}$ & $\begin{array}{c}0.0809^{* * *} \\
(0.0016)\end{array}$ & $\begin{array}{c}0.0806 * * * \\
(0.0016)\end{array}$ \\
\hline Kellogg's & $\begin{array}{c}0.0772 * * * \\
(0.0015)\end{array}$ & $\begin{array}{c}0.0769^{* * *} \\
(0.0016)\end{array}$ & $\begin{array}{c}0.0743^{* * *} \\
(0.0017)\end{array}$ & $\begin{array}{c}0.0736^{* * *} \\
(0.0018)\end{array}$ & $\begin{array}{c}0.0820^{* * *} \\
(0.0015)\end{array}$ & $\begin{array}{c}0.0817^{* * *} \\
(0.0016)\end{array}$ \\
\hline General Mills $\times$ Imitated & & & & & $\begin{array}{c}-0.0144 * * * \\
(0.0034)\end{array}$ & $\begin{array}{c}-0.0153 * * * \\
(0.0033)\end{array}$ \\
\hline Kellogg's $\times$ Imitated & & & & & $\begin{array}{c}-0.0508^{* * *} \\
(0.0035)\end{array}$ & $\begin{array}{c}-0.0516^{* * *} \\
(0.0034)\end{array}$ \\
\hline Constant & $\begin{array}{c}0.0136^{* * *} \\
(0.0037)\end{array}$ & $\begin{array}{c}0.0150 * * * \\
(0.0037)\end{array}$ & $\begin{array}{c}0.0031 \\
(0.0041) \\
\end{array}$ & $\begin{array}{c}0.0043 \\
(0.0041) \\
\end{array}$ & $\begin{array}{c}0.0138^{* * *} \\
(0.0036)\end{array}$ & $\begin{array}{c}0.0151^{* * *} \\
(0.0037)\end{array}$ \\
\hline Obs. & 2,480 & 2,480 & 2,480 & 2,480 & 2,480 & 2,480 \\
\hline R-squared & 0.5552 & 0.5487 & 0.5298 & 0.5197 & 0.5815 & 0.5750 \\
\hline
\end{tabular}

Note: Robust standard errors in parentheses. Quarter-by-year dummies are included but not re-

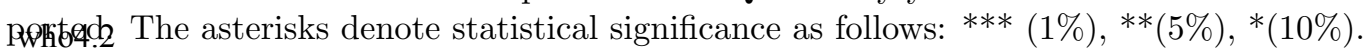


Table 3.11: Consumer surplus under the baseline and counterfactual scenarios

\begin{tabular}{cccc}
\hline Price zone & $\begin{array}{c}(1) \\
\text { Baseline }\end{array}$ & $\begin{array}{c}(2) \\
\text { Counterfactual }\end{array}$ & $\begin{array}{c}(3)=(1)-(2) \\
\text { Gain }\end{array}$ \\
\hline High & $\$ 3,460,443$ & $\$ 2,919,139$ & $\$ 541,304$ \\
Medium & $\$ 4,807,168$ & $\$ 4,118,330$ & $\$ 688,838$ \\
Low & $\$ 1,615,381$ & $\$ 1,371,808$ & $\$ 243,573$ \\
Cub fighter & $\$ 1,442,519$ & $\$ 1,240,559$ & $\$ 201,960$ \\
\hline Total & $\$ 11,325,512$ & $\$ 9,649,837$ & $\$ 1,675,675$ \\
\hline
\end{tabular}

Note: In the baseline scenario I calculate consumer surplus given the set of brands observed in the

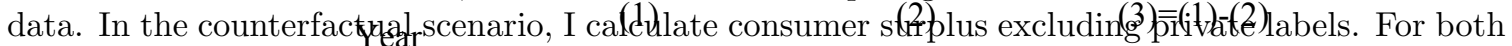

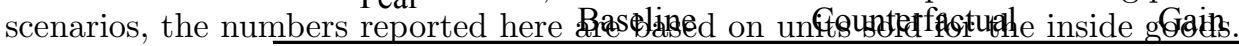

\begin{tabular}{cccc}
\hline 1989 & $\$ 422,364$ & $\$ 415,999$ & $\$ 6,365$ \\
1990 & $\$ 1,621,959$ & $\$ 1,476,021$ & $\$ 145,938$ \\
1991 & $\$ 1,808,863$ & $\$ 1,523,397$ & $\$ 285,466$ \\
1992 & $\$ 1,791,964$ & $\$ 1,369,243$ & $\$ 422,721$ \\
1993 & $\$ 1,511,766$ & $\$ 1,312,714$ & $\$ 199,052$ \\
1994 & $\$ 1,511,497$ & $\$ 1,265,438$ & $\$ 246,058$ \\
1995 & $\$ 723,201$ & $\$ 602,718$ & $\$ 120,482$ \\
1996 & $\$ 1,462,927$ & $\$ 1,279,470$ & $\$ 183,456$ \\
1997 & $\$ 470,973$ & $\$ 404,836$ & $\$ 66,137$ \\
\hline Total & $\$ 11,325,512$ & $\$ 9,649,837$ & $\$ 1,675,675$ \\
\hline
\end{tabular}

\begin{tabular}{ccccc}
\hline \multirow{2}{*}{ Price zone } & $(1)$ & $(2)$ & \multicolumn{2}{c}{ Gain: $(3)=(1)-(2)$} \\
\cline { 4 - 5 } & Baseline & Counterfactual & Value & Percentage \\
\hline High & $\$ 2,084,633$ & $\$ 927,451$ & $\$ 1,157,182$ & $55.5 \%$ \\
Medium & $\$ 2,901,569$ & $\$ 1,353,464$ & $\$ 1,548,104$ & $53.4 \%$ \\
Low & $\$ 968,156$ & $\$ 435,097$ & $\$ 533,059$ & $55.1 \%$ \\
Cub fighter & $\$ 886,581$ & $\$ 429,475$ & $\$ 457,106$ & $51.6 \%$ \\
\hline
\end{tabular}

\begin{tabular}{ccccc}
\hline \multirow{2}{*}{ Price zone } & $(1)$ & $(2)$ & \multicolumn{2}{c}{ Gain: $(3)=(1)-(2)$} \\
\cline { 4 - 5 } & Baseline & Counterfactual & Value & Percentage \\
\hline High & $\$ 1,983,735$ & $\$ 1,313,814$ & $\$ 669,921$ & $33.8 \%$ \\
Medium & $\$ 2,741,523$ & $\$ 1,807,747$ & $\$ 933,776$ & $34.1 \%$ \\
Low & $\$ 945,437$ & $\$ 641,719$ & $\$ 303,719$ & $32.1 \%$ \\
Cub fighter & $\$ 804,809$ & $\$ 521,070$ & $\$ 283,740$ & $35.3 \%$ \\
\hline
\end{tabular}


Figure 3.1: Example of cereal product characteristics

\section{Kellogg's \\ Frosted Flakes ${ }^{\circledR}$}

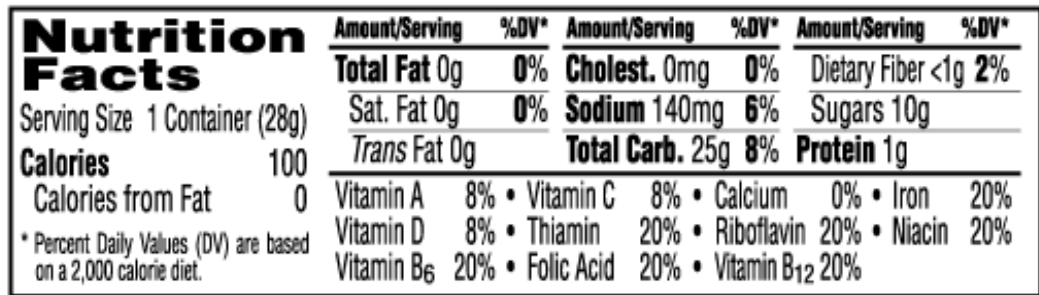

INGREDIENTS: MILLED CORN, SUGAR, CONTAINS $2 \%$ OR LESS OF MALT FLAVOR, SALT, BHT FOR FRESHNESS.

VITAMINS AND MINERALS: IRON, VITAMIN C (ASCORBIC ACID AND SODIUM ASCORBATE), NIACINAMIDE, VITAMIN B6

(PYRIDOXINE HYDROCHLORIDE), VITAMIN B 2 (RIBOFLAVIN), VITAMIN B (THIAMIN HYDROCHLORIDE), VITAMIN A

PALMITATE, FOLIC ACID, VITAMIN D, VITAMIN B 12 .

CORN USED IN THIS PRODUCT MAY CONTAIN TRACES OF SOYBEANS.

NLI\#09918 


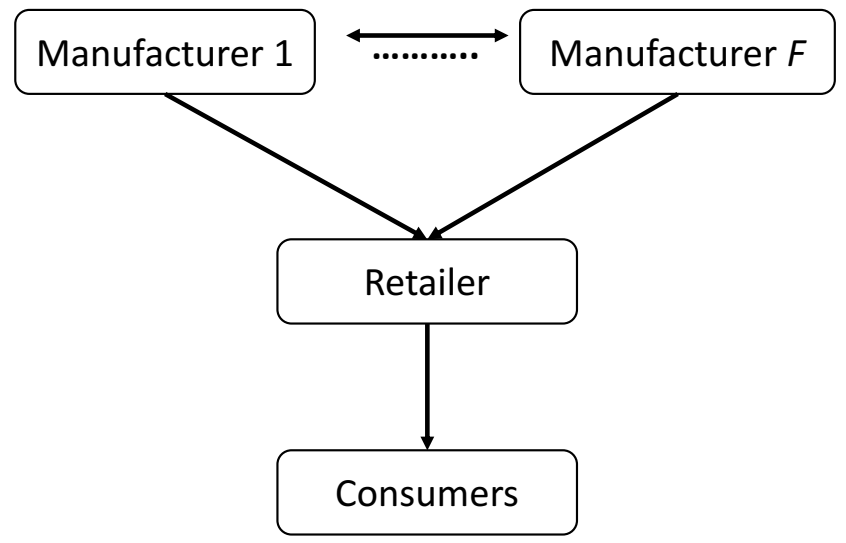

\section{Bibliography}

Ailawadi, K. L. And B. Harlam (2004): "An Empirical Analysis of the Determinants of Retail Margins: The Role of Store-Brand Share," Journal of Marketing, $68,147-165$.

Bergès-Sennou, F. (2006): "Store Loyalty, Bargaining Power and the Private Label Production Issue," European Review of Agricultural Economics, 33, 315-335.

Bergès-Sennou, F., F. Bontems, and V. Rèquillart (2004): "Economics of Private Labels: A Survey of Literature," Journal of Agricultural \&3 Food Industrial Organization, 2, 1-23.

Berry, S. T. (1994): "Estimating Discrete-Choice Models of Product Differentiation," The RAND Journal of Economics, 25, 242-262.

Berry, S. T., J. Levinsohn, And A. Pakes (1995): "Automobile Prices in Market Equilibrium," Econometirca, 63, 841-890. 
Bontemps, C., V. Orozco, and V. Requillart (2008): "Private Labels, National Brands and Food Prices," Review of Industrial Organization, 33, 1-22.

Bontems, P., S. Monier-Dilhan, and V. Requillart (1999): "Strategic Effects of Private Labels," European Review of Agricultural Economics, 26, 147-165.

Chintagunta, P. K. (2002): "Investigating Category Pricing Behavior at a Retail Chain," Journal of Marketing Research, 39, 141-154.

Gabrielsen, T. S. and L. Sorgard (2007): "Private Labels, Price Rivalry, and Public Policy," European Economic Review, 51, 403-424.

Hausman, J. (1996): "Valuation of New Goods under Perfect and Imperfect Competition," in The Economics of New Goods, ed. by T. Bresnahan and R. Gordon, University of Chicago Press.

Hausman, J. And G. Leonard (2002): "The Competitive Effects of a New Product Introduction: A Case Study," The Journal of Industrial Economics, 50, 237-263.

Hoch, S. J. and S. BanerJi (1993): "When Do Private Labels Succeed?" Sloan Management Review, 34, 57-67.

Meza, S. And K. Sudhir (2010): "Do Private Labels Increase Retailer Bargaining Power?" Quantitative Marketing and Economics, 8, 333-363.

Mills, D. E. (1995): "Why Retailers Sell Private Labels," Journal of Economics \&3 Management Strategy, 4, 509-528.

Narasimhan, C. And R. T. Wilcox (1998): "Private Labels and the Channel Relationship: A Cross-Category Analysis," The Journal of Business, 71, 573-600. 
Nevo, A. (2000): "A Practitioner's Guide To Estimation Of Random-Coefficients Logit Models Of Demand," Journal of Economics \& Management Strategy, 9, 513548.

(2001): "Measuring Market Power In The Ready-To-Eat Cereal Industry," Econometrica, 69, 307-342.

Pauwels, K. and S. SRinivasan (2004): "Who Benefits From Store Brand Entry?" Marketing Science, 23, 364-390.

Raju, J. S., R. Sethuraman, and S. Ghar (1995): "The Introduction and Performance of Store Brands," Managment Science, 41, 957-978.

Sayman, S., S. J. H, And J. S. RaJu (2002): "Positioning of Store Brands," Marketing Science, 21, 378-397.

Schmeltzer, J. (2007): "Roundy's Joins Chicago Grocery Fray: Milwaukee Chain Plains Rapid Expansion Here," http:// articles . chicagotribune. com/2007-02-13/business/0702130239_1_ chicago-market-fresh-store-concept-market-share.

SudHIR, K. (2001): "Structureal Analysis of Manufacturer Pricing in the Presence of a Strategic Retailer," Marketing Science, 20, 244-264.

TrajtenberG, M. (1989): "The Welfare Analysis of Product Innovations, with an Application to Computed Tomography Scanners," The Journal of Political Economiy, 97, 444-479.

Villas-Boas, J. M. and Y. ZhaO (2005): "Retailer, Manufacturers, and Individual Consumers: Modeling the Supply Side in the Ketchup Marketplace," Journal of Marketing Research, 42, 83-95. 
Wooldridge, J. M. (1995): "Score diagnostics for linear models estimated by two stage least squares," in Advances in Econometrics and Quantitative Economics: Essays in Honor of Professor C. R. Rao, ed. by G. S. Maddala, P. C. B. Phillips, and T. N. Srinivasan, Oxford: Oxford: Blackwell, 66-87. 


\section{Appendix A}

\section{Appendix for Chapter 1}

Proof of $w=c_{N B}$ in the case of perfect information: To show that $w=c_{N B}$ under perfect information, we write the retailer's profit maximization problem as:

$$
\max \Pi\left(p_{N B}, p_{P L}\right)=(1+\delta)\left[\left(p_{N B}-w\right) D_{N B}\left(p_{N B}, p_{P L}\right)+\left(p_{P L}-c_{P L, H}\right)\left(p_{N B}, p_{P L}\right)\right] .
$$

The retailer does not have incentive to change the retail prices through the two periods since the information about the product quality is perfect. The first-order conditions to the associated problem are:

$$
\begin{aligned}
& D_{N B}\left(p_{N B}, p_{P L}\right)+\left(p_{N B}-w\right) D_{N B, 1}\left(p_{N B}, p_{P L}\right)+\left(p_{P L}-c_{P L, H}\right) D_{P L, 1}\left(p_{N B}, p_{P L}\right)=0, \\
& D_{P L}\left(p_{N B}, p_{P L}\right)+\left(p_{N B}-w\right) D_{N B, 2}\left(p_{N B}, p_{P L}\right)+\left(p_{P L}-c_{P L, H}\right) D_{P L, 2}\left(p_{N B}, p_{P L}\right)=0,
\end{aligned}
$$

where $D_{i j}(\cdot)$ represents the marginal effect from the good $j$ 's price on the good $i$ 's demand. Moreover, the negotiated wholesale price on the good 1 must maximize the 
joint profit between the two firms, that is

$$
\begin{aligned}
w^{*} & =\operatorname{argmax} \quad \Pi_{\text {joint }} \\
& =(1+\delta)\left[\left(p_{N B}(w)-c_{N B}\right] D_{N B}\left(p_{N B}(w), p_{P L}(w)\right)+\left(p_{P L}(w)-c_{P L, H}\right) D_{P L}\left(p_{N B}(w), p_{P L}(w)\right)\right] .
\end{aligned}
$$

The solution to above problem must satisfy the first-order condition

$$
\begin{aligned}
& p_{N B}^{\prime}(w)\left[D_{N B}\left(p_{N B}, p_{P L}\right)+\left(p_{N B}-w\right) D_{N B, 1}\left(p_{N B}, p_{P L}\right)\right. \\
& \left.\quad+\left(p_{P L}-c_{P L, H}\right) D_{P L, 1}\left(p_{N B}, p_{P L}\right)\right] \\
& \quad+p_{P L}^{\prime}(w)\left[D_{P L}\left(p_{N B}, p_{P L}\right)+\left(p_{N B}-w\right) D_{N B, 2}\left(p_{N B}, p_{P L}\right)\right. \\
& \left.\quad+\left(p_{P L}-c_{P L, H}\right) D_{P L, 2}\left(p_{N B}, p_{P L}\right)\right]=0 .
\end{aligned}
$$

Both $p_{N B}^{\prime}(w)$ and $p_{P L}^{\prime}(w)$ are positive. In order to satisfy the first-order conditions (A.1.1)-(A.1.3) at the same time, the wholesale price $w$ has to be equal to $c_{N B}$. Q.E.D.

Proof of $w=c_{N B}$ in the case of imperfect information: If the information about the quality of the good 2 (e.g., the PL product) is imperfect, an incentive compatibility constraint is taken into account, the retailer's (constrained) profit maximization problem is:

$\max \Pi\left(p_{N B}^{t}, p_{P L}^{t}\right)=\Pi_{N B}^{1}\left(p_{N B}^{t}, p_{P L}^{t}\right)+\Pi_{P L}^{1}\left(p_{N B}^{t}, p_{P L}^{t}\right)+\delta\left[\Pi_{N B}^{2}\left(p_{N B}^{t}, p_{P L}^{t}\right)+\Pi_{P L}^{2}\left(p_{N B}^{t}, p_{P L}^{t}\right)\right]$

s.t. : $\quad \Pi\left(p_{N B}^{t}, p_{P L}^{t}\right) \geq \widetilde{\Pi}\left(p_{N B}^{t}, p_{P L}^{t}\right)$

Where $\widetilde{\Pi}\left(p_{N B}^{t}, p_{P L}^{t}\right)$ represents the retailer's profit from selling the low-quality product and it is independent of the second period prices since all consumers will refuse to buy from this retailer in period 2 if they detected the inferior good at the end of period 1. A Lagrange function to the associated problem can be written as:

$$
\mathscr{L}\left(p_{N B}^{t}, p_{P L}^{t}\right)=(1+\lambda) \Pi\left(p_{N B}^{t}, p_{P L}^{t}\right)-\lambda \widetilde{\Pi}\left(p_{N B}^{t}, p_{P L}^{t}\right)
$$


And the first-order conditions are:

$$
\begin{gathered}
\mathscr{L}_{1}^{1}(\cdot)=(1+\lambda) \frac{\partial \Pi\left(p_{N B}^{t}, p_{P L}^{t}\right)}{\partial p_{N B}^{1}}-\lambda \frac{\partial \widetilde{\Pi}\left(p_{N B}^{t}, p_{P L}^{t}\right)}{\partial p_{N B}^{1}}=0, \\
\mathscr{L}_{2}^{1}(\cdot)=(1+\lambda) \frac{\partial \Pi\left(p_{N B}^{t}, p_{P L}^{t}\right)}{\partial p_{P L}^{1}}-\lambda \frac{\partial \widetilde{\Pi}\left(p_{N B}^{t}, p_{P L}^{t}\right)}{\partial p_{P L}^{1}}=0, \\
\mathscr{L}_{1}^{2}(\cdot)=(1+\lambda) \frac{\partial \Pi\left(p_{N B}^{t}, p_{P L}^{t}\right)}{\partial p_{N B}^{2}}, \\
\mathscr{L}_{2}^{2}(\cdot)=(1+\lambda) \frac{\partial \Pi\left(p_{N B}^{t}, p_{P L}^{t}\right)}{\partial p_{P L}^{2}} .
\end{gathered}
$$

Moreover, the joint profit maximization problem is:

$$
\begin{array}{ll}
\max & \Pi\left(p_{N B}^{t *}(w), p_{P L}^{t *}(w)\right) \\
\text { s.t. : } & \Pi\left(p_{N B}^{t *}(w), p_{P L}^{t *}(w)\right) \geq \widetilde{\Pi}\left(p_{N B}^{t *}(w), p_{P L}^{t *}(w)\right) .
\end{array}
$$

The Lagrange function to the associated problem is:

$$
\mathscr{L}\left(p_{N B}^{t *}(w), p_{P L}^{t *}(w)\right)=(1+\lambda) \Pi\left(p_{N B}^{t *}(w), p_{P L}^{t *}(w)\right)-\lambda \widetilde{\Pi}\left(p_{N B}^{t *}(w), p_{P L}^{t *}(w)\right) .
$$

The first-order condition is:

$$
\begin{aligned}
\mathscr{L}^{\prime}(\cdot) & =(1+\delta)\left[\frac{\partial \Pi(\cdot)}{\partial p_{N B}^{1 *}} p_{N B}^{1 *^{\prime}}+\frac{\partial \Pi(\cdot)}{\partial p_{P L}^{1 *}} p_{P L}^{1 *^{\prime}}+\frac{\partial \Pi(\cdot)}{\partial p_{N B}^{2 *}} p_{N B}^{2 *^{\prime}}+\frac{\partial \Pi(\cdot)}{\partial p_{P L}^{2 *}} p_{P L}^{2 *^{\prime}}\right] \\
& -\lambda\left[\frac{\partial \widetilde{\Pi}(\cdot)}{\partial p_{N B}^{1 *}} p_{N B}^{1 *^{\prime}}+\frac{\partial \widetilde{\Pi}(\cdot)}{\partial p_{P L}^{1 *}} p_{P L}^{1 *^{\prime}}\right]=0 .
\end{aligned}
$$

Again, to satisfy the first-order conditions (A.1.4)-(A.1.7) and (A.1.8) at the same time, $w$ has to be equal to $c_{N B}$. An alternative thinking is that, the wholesale prices can be negotiated for two periods at the beginning of period 1, i.e., $w^{1}$ and $w^{2}$. This will not change the result and we can eventually obtain $w^{1}=w^{2}=c_{N B}$ in efficiency. 
Q.E.D.

Proof of Lemma 1.1: Substituting $p_{N B}^{*}$ and $p_{P L}^{*}$ into (1.5) and (1.6) in the text and making them to be strictly positive yields that $c_{N B}<c_{N B}^{b}$ from $D_{N B}\left(p_{N B}^{*}, p_{P L}^{*}\right)$ and $c_{N B}>c_{N B}^{c}$ from $D_{P L}\left(p_{N B}^{*}, p_{P L}^{*}\right)$.

In addition, $c_{N B}^{b}-c_{N B}^{c}=\frac{\left(s_{N B}-s_{P L, H}\right)\left(c_{P L, H}^{a}-c_{P L, H}\right)}{s_{P L, H}-s_{P L, L}}$, which is strictly positive since $c_{P L, H}<c_{P L, H}^{a}$. Similar to $c_{N B}^{a}-c_{N B}^{b}=c_{P L, H}^{a}-c_{P L, H}>0$. Moreover, $c_{N B}^{c}-c_{P L, H}=$ $\frac{\left(s_{N B}-s_{P L, H}\right)\left(c_{P L, H}-c_{P L, L}\right)}{s_{P L, H}-s_{P L, L}}>0$. Q.E.D.

Proof of Proposition 1.1: The retailer's bargaining payoff from (NB, PL) is $\gamma \Pi_{N B, P L}+(1-\gamma) \Pi_{P L}^{*}-K=\gamma \Pi_{N B}^{*}+(1-\gamma) \Pi_{P L}^{*}-K$ since $\Pi_{N B, P L}=\Pi_{N B}^{*}$ in this case. If the product line $(\mathrm{NB}, \mathrm{PL})$ is more profitable than $(\mathrm{NB}, 0)$, we have

$$
\gamma \Pi_{N B}^{*}+(1-\gamma) \Pi_{P L}^{*}-K \geq \gamma \Pi_{N B}^{*}, \quad \text { or } \quad(1-\gamma) \Pi_{P L}^{*}-K \geq 0
$$

This always holds since we have assumed that is very small.

On the other hand, if the product line $(\mathrm{NB}, \mathrm{PL})$ is more profitable than $(0, \mathrm{PL})$, we have:

$$
\gamma \Pi_{N B}^{*}+(1-\gamma) \Pi_{P L}^{*} \geq \gamma \Pi_{P L}^{*}, \quad \text { or } \quad \Pi_{N B}^{*} \geq \Pi_{P L}^{*}
$$

Substituting the equilibrium parameters into the above inequality yields:

$$
\begin{aligned}
& {\left[\bar{\theta}\left(s_{N B}-s_{P L, L}\right)-\left(c_{N B}-c_{P L, L}\right)\right]\left[\bar{\theta}-\frac{c_{N B}-c_{P L, L}}{s_{N B}-s_{P L, L}}\right]} \\
& \quad-\left[\bar{\theta}\left(s_{P L, H}-s_{P L, L}\right)-\left(c_{P L, H}-c_{P L, L}\right)\right]\left[\bar{\theta}-\frac{c_{P L, H}-c_{P L, L}}{s_{P L, H}-s_{P L, L}}\right] \geq 0 .
\end{aligned}
$$

The difference of the first brackets from the two terms in the inequality shows that: $c_{N B}^{b}-c_{N B}>0$. And the difference of the second brackets from the corresponding terms in the inequality shows that: $\frac{c_{N B}^{c}-c_{N B}}{s_{N B}-s_{P L, L}} \geq 0$. Therefore, $\Pi_{N B, P L} \geq \Pi_{P L}^{*}$. In addition, the demand for the PL product is zero in equilibrium since $c_{N B} \leq c_{N B}^{c}$ in this case. 
Q.E.D.

Proof of Proposition 1.2: The retailer's bargaining payoff from (NB, PL) is $\gamma \Pi_{N B, P L}+(1-\gamma) \Pi_{P L}^{*}-K=\Pi_{P L}^{*}-K$ since $\Pi_{N B, P L}=\Pi_{P L}^{*}$ in this case. Moreover, if $(0, \mathrm{PL})$ is more profitable than $(\mathrm{NB}, 0)$, we have $\Pi_{P L}^{*}-\gamma \Pi_{N B}^{*}-K \geq 0$. Moreover,

$$
\begin{aligned}
\Pi_{P L}^{*}-\Pi_{N B}^{*} & =\frac{1-\delta}{4}\left\{\left[\bar{\theta}\left(s_{P L, H}-s_{P L, L}-\left(c_{P L, H}-c_{P L, L}\right)\right]\left[\bar{\theta}-\frac{c_{P L, H}-c_{P L, L}}{s_{P L, H}-s_{P L, L}}\right]\right.\right. \\
& \left.-\left[\bar{\theta}\left(s_{N B}-s_{P L, L}\right)-\left(c_{N B}-c_{P L, L}\right)\right]\left[\bar{\theta}-\frac{c_{N B}-c_{P L, L}}{s_{N B}-s_{P L, L}}\right]\right\} \geq 0 .
\end{aligned}
$$

The difference of the first brackets from the two terms in the inequality shows that: $c_{N B}-c_{N B}^{b} \geq 0$. And the difference of the second brackets from the corresponding terms in the inequality shows that: $\frac{c_{N B}-c_{N B}^{c}}{s_{N B}-s_{P L, L}}>0$. Therefore, $\Pi_{P L} \geq \Pi_{N B}$ and thus $\Pi_{P L}^{*}-\gamma \Pi_{N B}^{*}-K \geq 0$ for $\gamma \in[0,1]$ and a very small $K$. Q.E.D.

Proof of Proposition 1.3: The retailer's bargaining payoff from (NB, PL) is $\gamma \Pi_{N B, P L}^{*}+(1-\gamma) \Pi_{P L}^{*}-K$. If $(\mathrm{NB}, \mathrm{PL})$ is more profitable than $(\mathrm{NB}, 0)$, we have: $\gamma \Pi_{N B, P L}^{*}+(1-\gamma) \Pi_{P L}^{*}-K \geq \gamma \Pi_{N B}^{*}$. In this inequality, the term $(1-\gamma) \Pi_{P L}^{*}-K$ is positive for a very small $K$. And $\Pi_{N B, P L}^{*}-\Pi_{N B}^{*}$ can be expressed as:

$$
\begin{aligned}
& \frac{1+\delta}{4} \frac{1}{s_{N B}-s_{P L, H}} \frac{1}{s_{N B}-s_{P L, L}} \frac{1}{s_{P L, H}-s_{P L, L}} \\
& \quad \times\left[c_{N B}\left(s_{P L, H}-s_{P L, L}\right)-c_{P L, H}\left(s_{N B}-s_{P L, L}\right)+c_{P L, L}\left(s_{N B}-s_{P L, H}\right)\right]^{2},
\end{aligned}
$$

which is positive. Therefore, $\gamma\left(\Pi_{N B, P L}^{*}-\Pi_{N B}^{*}\right)+(1-\gamma) \Pi_{P L}^{*}-K \geq 0$ for a very small $K$.

On the other hand, if (NB, PL) is more profitable than (0, PL), we have: $\gamma \prod_{N B, P L}^{*}+$ $(1-\gamma) \Pi_{P L}^{*}-K \geq \Pi_{P L}^{*}-K$, or $\Pi_{N B, P L}^{*} \geq \Pi_{P L}^{*} \cdot \Pi_{N B, P L}^{*}-\Pi_{P L}^{*}$ can be expressed as:

$$
\frac{1+\delta}{4} \frac{1}{s_{N B}-s_{P L, H}}\left[\bar{\theta}\left(s_{N B}-s_{P L, H}\right)-\left(c_{N B}-c_{P L, H}\right)\right]^{2},
$$


which is positive. Therefore, $\Pi_{N B, P L} \geq \Pi_{P L}^{*}$ in this case.

As a whole, the product line $(\mathrm{NB}, \mathrm{PL})$ is the most profitable one in this case. In addition, the demands for both $\mathrm{NB}$ and PL product are positive in equilibrium. Q.E.D.

Proof of Lemma 1.2: If the incentive compatibility constraint is non-binding, the multiplier $\lambda$ equals to zero (this comes from the supplementary slackness (1.11) in the text). Solving (1.9) and (1.10) in the text implies that the retail price on the PL product is $p_{P L}^{*}$ in both periods. Substituting this price into the non-binding incentive compatibility constraint $\Pi\left(p_{P L}^{1}, p_{P L}^{2}\right)-\widetilde{\Pi}\left(p_{P L}^{1}\right)>0$, we obtain: $c_{P L, H}<c_{P L, H}^{b}$. In addition, the equilibrium profit in this situation is identical to the perfect information one since $p_{P L}=p_{P L}^{*}$.

On the other hand, if the incentive compatibility constraint is binding, then $\lambda \geq 0$. Solving (1.9) and (1.10) in the text gives $p_{P L}^{1}=p_{P L}^{*}+\frac{\lambda}{2}\left(c_{P L, H}-c_{P L, L}\right)$ and $p_{P L}^{2}=p_{P L}^{*}$. We can solve for the expression of $\lambda$ by substituting these prices into the binding incentive compatibility constraint $\Pi\left(p_{P L}^{1}, p_{P L}^{2}\right)-\widetilde{\Pi}\left(p_{P L}^{1}\right)=0$ :

$\hat{\lambda}=-1-\frac{\delta \bar{\theta}^{2}\left(s_{P L, H}-s_{P L, L}\right)^{2}-2(1+\delta) \bar{\theta}\left(s_{P L, H}-s_{P L, L}\right)\left(c_{P L, H}-c_{P L, L}\right)+\delta\left(c_{P L, H}-c_{P L, L}\right)^{2}}{2\left(c_{P L, H}-c_{P L, L}\right)^{2}}$.

For $\hat{\lambda} \geq 0$ we have: $c_{P L, H}^{b} \leq c_{P L, H}<c_{P L, H}^{a}$. In addition, the equilibrium profit from $(0$, $\mathrm{PL})$ in this situation is $\hat{\Pi}_{P L}=\Pi_{P L}^{*}=\frac{\hat{\lambda}^{2}}{4} \frac{\left(c_{P L, H}-c_{P L, L}\right)^{2}}{s_{P L, H}-s_{P L, L}}$. Q.E.D.

Proof of Lemma 1.3: The equilibrium profit from the product line (0, PL) if $c_{P L, H}^{b} \leq c_{P L, H}<c_{P L, H}^{a}$ is: $\hat{\prod}_{P L}=\Pi_{P L}^{*}=\frac{\hat{\lambda}^{2}}{4} \frac{\left(c_{P L, H}-c_{P L, L}\right)^{2}}{s_{P L, H}-s_{P L, L}}$. The expression of $\hat{\lambda}$ is given in Lemma 1.2. If $\hat{\Pi}_{P L}>0$, we have:

$$
\hat{\lambda}<\left|\sqrt{1+\delta} \cdot \frac{\bar{\theta}\left(s_{P L, H}-s_{P L, L}\right)-\left(c_{P L, H}-c_{P L, L}\right)}{c_{P L, H}-c_{P L, L}}\right| .
$$

The absolute sign in condition (A.1.9) can be removed since $\bar{\theta}\left(s_{P L, H}-s_{P L, L}\right)-\left(c_{P L, H}-\right.$ 
$\left.c_{P L, L}\right)>0$ (Assumption 1.2) and $c_{P L, H}>c_{P L, L}$. Thus (A.1.9) becomes:

$$
0<\hat{\lambda}<\sqrt{1+\delta} \cdot \frac{\bar{\theta}\left(s_{P L, H}-s_{P L, L}\right)-\left(c_{P L, H}-c_{P L, L}\right)}{c_{P L, H}-c_{P L, L}} .
$$

The inequality can be solved:

$$
c_{P L, H}<c_{P L, H}^{a} \quad \text { or } \quad c_{P L, H}>\frac{\delta}{(\sqrt{1+\delta}-1)^{2}} \bar{\theta}\left(s_{P L, H}-s_{P L, L}\right)+c_{P L, L},
$$

which can be refined to $c_{P L, H}<c_{P L, H}^{a}$ since $\frac{\delta}{(\sqrt{1+\delta}-1)^{2}}>1$. Therefore, for any $c_{P L, H} \in$ $\left[c_{P L, H}^{b}, c_{P L, H}^{a}\right), \hat{\Pi}_{P L}>0$. Q.E.D.

Proof of Proposition 1.4: If $c_{P L, H}<c_{P L, H}^{b}$, the retailer's bargaining payoff from selling the NB product is $\gamma \Pi_{N B}^{*}+(1-\gamma) \Pi_{P L}^{*}-K$ which is same as the one in case 1 . Moreover, the retailer's payoff $(0, \mathrm{PL})$ is $\Pi_{P L}^{*}-K$. According to Proposition 1.1, the profit from $(\mathrm{NB}, \mathrm{PL})$ is greater than that from $(0, \mathrm{PL})$ in the case of $c_{N B} \leq c_{N B}^{c}$.

On the other hand, if $c_{P L, H}^{b} \leq c_{P L, H}<c_{P L, H}^{a}$, the retailer's bargaining payoff from selling the NB product is $\gamma \Pi_{N B}^{*}+(1-\gamma) \hat{\Pi}_{P L}-K$, and the second term is strictly positive (see Lemma 1.3). This bargaining payoff is greater than the profit from $(0, \mathrm{PL})$ if $\Pi_{N B}^{*} \geq \hat{\Pi}_{P L}$. With the help of Proposition 1.1, that is true since $\Pi_{N B}^{*}>\Pi_{P L}^{*}>\hat{\Pi}_{P L}$, note that $\Pi_{N B}^{*}>\Pi_{P L}^{*}$ comes from Proposition 1.1 and, $\Pi_{P L}^{*}>\hat{\Pi}_{P L}$ comes from Lemma 1.2. Q.E.D.

Proof of Lemma 1.5: We first discuss the situation that the demand for the NB product is zero in period 1, then the second constraint in the associated profit maximization problem is binding and $\lambda_{2} \geq 0$. If the incentive compatibility constraint is non-binding, then $\lambda_{1}=0$, solving the first-order conditions (1.12) and (1.13) in the text yields: $p_{N B}^{1}=p_{N B}^{*}-\frac{\lambda_{2}}{2}$, and $p_{P L}^{1}=p_{P L}^{*}$. Substituting these retail prices into the second (binding) constraint $D_{N B}^{1}\left(p_{N B}^{1}, p_{P L}^{1}\right)=0$ gives: $\hat{\lambda}_{2}=c_{N B}-c_{N B}^{b} \geq 0$. Plugging $p_{N B}^{1}, p_{P L}^{1}$ and $\hat{\lambda}_{2}$ into the (non-binding) incentive compatibility constraint 
gives $c_{P L, H}<c_{P L, H}^{b}$ and the equilibrium demand for the NB product is zero in this situation.

If the incentive compatibility is binding, $\lambda_{1} \geq 0$, solving the first-order conditions (1.12) and (1.13) gives that $p_{N B}^{1}=p_{N B}^{*}-\frac{\lambda_{2}}{2}$ and $p_{P L}^{1}=p_{P L}^{*}+\frac{\lambda_{1}\left(c_{P L, H}-c_{P L, L}\right)}{2}$. Substituting these retail prices into the incentive compatibility constraint yields: $\frac{\left(c_{P L, H}-c_{P L, L}\right)\left(c_{P L, H}^{a}-c_{P L, H}\right)}{s_{P L, H}-s_{P L, L}}+\Pi_{P L}^{2 *}$, which is positive. This implies that the incentive compatibility constraint is always slack and contradicts $\lambda_{1} \geq 0$.

If the demand for the NB product is positive in period 1, the second constraint in the associated profit maximization problem is non-binding, i.e., $\lambda_{2}=0$. If the incentive compatibility constraint is also non-binding, $\lambda_{1}=0$. The first period retail price is same as the perfect information one. Accordingly, the demand for the NB product is zero in equilibrium, which contradicts the non-binding second constraint.

On the other hand, if the incentive compatibility constraint is binding, $\lambda_{1} \geq 0$, the expression of $\lambda_{1}$ can be derived as:

$$
\begin{aligned}
\hat{\lambda}_{1}= & \frac{s_{P L, H}-s_{P L, L}}{\left(s_{N B}-s_{P L, L}\right)\left(c_{P L, H}-c_{P L, L}\right)}\left(c_{N B}-c_{N B}^{c}\right) \\
& -\frac{\delta}{2} \frac{\left(s_{N B}-s_{P L, H}\right)\left(c_{P L, H}^{a}-c_{P L, H}\right)^{2}}{\left(s_{N B}-s_{P L, L}\right)\left(c_{P L, H}-c_{P L, L}\right)^{2}} .
\end{aligned}
$$

Substituting $\hat{\lambda}_{1}$ into the (non-binding) second constraint provides:

$$
\hat{\lambda}_{1}>\frac{c_{N B}-c_{N B}^{b}}{c_{P L, H}-c_{P L, L}}
$$

Solving the inequality in (A.1.12) with the expression of $\hat{\lambda}_{1}$ we can find that $c_{N B}^{b} \leq$ $c_{N B}<c_{N B}^{d}$. Moreover, $c_{N B}^{d} \geq c_{N B}^{b}$ if $c_{P L, H} \geq c_{P L, H}^{b}$. Therefore, the demand for the NB product in the product line $(\mathrm{NB}, \mathrm{PL})$ is positive in period 1 if $c_{P L, H}^{b} \leq c_{P L, H}<c_{P L, H}^{a}$ and $c_{N B}^{b} \leq c_{N B}<c_{N B}^{d}$. Q.E.D.

Proof of Proposition 1.5: If $c_{P L, H}<c_{P L, H}^{b}$, the retailer will sell only the PL 
product in equilibrium and obtain a profit $\Pi_{P L}^{*}$. With the help of Proposition 1.2, this profit is greater than that from selling the NB product although the latter is improved by a positive disagreement payoff. Therefore the retailer in this situation will choose the product line $(0, \mathrm{PL})$ in equilibrium.

If $c_{P L, H}^{b} \leq c_{P L, H}<c_{P L, H}^{a}$ and $c_{N B}^{b} \leq c_{N B}<c_{N B}^{d}$, the joint profit from the product line (NB, PL) is $\hat{\Pi}_{N B, P L}=\Pi_{N B, P L}^{1}\left(p_{N B}^{1}, p_{P L}^{1}, \hat{\lambda}_{1}\right)+\Pi_{P L}^{2 *}$. If the product line (NB, PL) is more profitable than $(0, \mathrm{PL})$, we have: $\hat{\Pi}_{N B, P L}^{1}\left(p_{N B}^{1}, p_{P L}^{1}, \hat{\lambda}_{1}\right)-\hat{\Pi}_{P L}^{1} \geq 0$. Further,

$$
\hat{\Pi}_{N B, P L}^{1}\left(p_{N B}^{1}, p_{P L}^{1}, \hat{\lambda}_{1}\right)-\left.\hat{\Pi}_{P L}^{1}\right|_{c_{N B}=c_{N B}^{*}}=\frac{1}{4} \frac{\left(c_{P L, H}-c_{P L, L}\right)^{2}}{s_{N B}-s_{P L, L}} \lambda^{2}>0,
$$

and

$\hat{\Pi}_{N B, P L}^{1}\left(p_{N B}^{1}, p_{P L}^{1}, \hat{\lambda}_{1}\right)-\left.\hat{\Pi}_{P L}^{1}\right|_{c_{N B}=c_{N B}^{d}}=\frac{1}{4} \frac{1}{s_{N B}-s_{P L, L}}\left(c_{N B}^{b}-c_{N B}\right)^{2}+\frac{1}{4} \frac{\left(c_{P L, H}-c_{P L, L}\right)^{2}}{s_{N B}-s_{P L, L}} \lambda^{2}>0$.

In addition, the value of $\hat{\Pi}_{N B, P L}^{1}\left(p_{N B}^{1}, p_{P L}^{1}, \hat{\lambda}_{1}\right)-\hat{\Pi}_{P L}^{1}$ is monotonically decreasing in $c_{N B} \in\left[c_{N B}^{b}, c_{N B}^{d}\right)$ and always positive in this range. In addition, this value achieves the "bottom" if $c_{N B}=c_{N B}^{d}$. However, since $c_{N B}<c_{N B}^{d}$ instead of $c_{N B} \leq c_{N B}^{d}$, the value only approaches rather equals to the "bottom". As a result, $\forall c_{N B} \in\left[c_{N B}^{b}, c_{N B}^{d}\right)$ : $\Pi_{N B, P L}^{1}\left(p_{N B}^{1}, p_{P L}^{1}, \hat{\lambda}_{1}\right)-\hat{\Pi}_{P L}^{1}>0$, which implies that the product line (NB, PL) is more profitable than $(0, \mathrm{PL})$ in this situation.

On the other hand, if the product line $(\mathrm{NB}, \mathrm{PL})$ is more profitable than $(\mathrm{NB}, 0)$, we have:

$$
\gamma\left\{\left[\hat{\Pi}_{N B, P L}^{1}\left(p_{N B}^{1}, p_{P L}^{1}, \hat{\lambda}_{1}\right)-\Pi_{N B}^{1 *}\right]+\left[\Pi_{P L}^{2 *}-\Pi_{N B}^{2 *}\right]\right\}+(1-\gamma) \Pi_{P L}-K \geq 0 .
$$

The value of $\left.\Pi_{P L}^{2 *}-\Pi_{N B}^{2 *}\right]$ is positive, which is proved in Proposition 1.2. And the value of $\hat{\Pi}_{N B, P L}^{1}\left(p_{N B}^{1}, p_{P L}^{1}, \hat{\lambda}_{1}\right)-\Pi_{N B}^{1 *}$ is monotonically increasing from $c_{N B}^{b}$ to $c_{N B}^{d}$ and 
$\forall c_{N B} \in\left[c_{N B}^{b}, c_{N B}^{d}\right): \hat{\Pi}_{N B, P L}^{1}\left(p_{N B}^{1}, p_{P L}^{1}, \hat{\lambda}_{1}\right)-\Pi_{N B}^{1 *}>0$, which implies that the product line $(\mathrm{NB}, \mathrm{PL})$ is more profitable than $(\mathrm{NB}, 0)$.

Finally, if $c_{P L, H}^{b} \leq c_{P L, H}<c_{P L, H}^{a}$ but $c_{N B}^{d} \leq c_{N B}<c_{N B}^{a}$, the retailer will not diversify its product line by carrying the NB product. Therefore, it will make decision between $(\mathrm{NB}, 0)$ and $(0, \mathrm{PL})$. If the latter is more profitable, we have $\hat{\Pi}_{P L} \geq \gamma \Pi_{N B}^{*}+(1-\gamma) \hat{\Pi}_{P L}$ or $\hat{\Pi}_{P L} \geq \Pi_{N B}^{*}$. Moreover, $\hat{\Pi}_{P L}$ is independent of $c_{N B}$ and $\Pi_{N B}^{*}$ is decreasing in $c_{N B} \in$ $\left[c_{N B} b, c_{N B}^{a}\right)$. Namely, the value of $\Pi_{N B}^{*}$ achieves the highest level if $c_{N B}=c_{N B}^{b}$. In this situation, $\hat{\Pi}_{P L} \geq \Pi_{N B}^{*} \mid c_{N B}=c_{N B}^{b}$ can be expressed as:

$$
(1+\delta) \frac{s_{N B}-s_{P L, H}}{s_{N B}-s_{P L, L}}\left(c_{P L, H}^{a}-c_{P L, H}\right)^{2} \leq \hat{\lambda}^{2}\left(c_{P L, H}-c_{P L, L}\right)^{2}
$$

With the expression of $\hat{\lambda}$, it can be found that the above inequality always holds if $c_{P L, H}<c_{P L, H}^{a}$, which implies that $\Pi_{N B}^{*}$ is always smaller than $\hat{\Pi}_{P L}$ for $c_{N B} \in\left[c_{N B}^{b}, c_{N B}^{a}\right)$. Q.E.D.

Proof of Corollary 1.1: The difference between $P_{N B, P L}$ and $P_{P L}$ can be expressed as:

$$
\begin{gathered}
\frac{1}{2} \frac{1}{s_{P L, H}-s_{P L, L}}\left[-\delta \bar{\theta}^{2}\left(s_{P L, H}-s_{P L, L}\right)^{2}+2(1+\delta) \bar{\theta}^{2}\left(s_{P L, H}-s_{P L, L}\right)\left(c_{P L, H}-c_{P L, L}\right)\right. \\
\left.-(2+\delta)\left(c_{P L, H}-c_{P L, L}\right)^{2}\right] .
\end{gathered}
$$

The inequality can be solved: $c_{P L, H}^{b}<c_{P L, H}<c_{P L, H}^{a}$. This condition on is same as that in the case of brand stretching, Therefore, $P_{N B, P L}>P_{P L}$ in the presence of brand stretching. Q.E.D.

Proof of Corollary 1.2: Recall the price distortion on the PL product in the product line $(\mathrm{NB}, \mathrm{PL})$ and $(0, \mathrm{PL})$ are respectively

$$
\rho_{N B, P L}=\frac{\hat{\lambda}_{1}}{2}\left(c_{P L, H}-c_{P L, H L}\right),
$$


and

$$
\rho_{P L}=\frac{\hat{\lambda}}{2}\left(c_{P L, H}-c_{P L, L}\right)
$$

According to the expression of $\hat{\lambda}_{1}$, it is increasing in $c_{N B} \in\left[c_{N B}^{b}, c_{N B}^{d}\right)$. if $c_{N B}=c_{N B}^{d}$, it can be found that $\hat{\lambda}_{1}=\hat{\lambda}$. This means that the highest level of $\rho_{N B, P L}$ approaches $\rho_{P L}$ for $c_{N B} \in\left[c_{N B}^{b}, c_{N B}^{d}\right)$, but these two cannot be equal since $c_{N B}$ is approaching $c_{N}^{d}$ in this case. Therefore, we can see that the price distortion on the PL product is smaller in the product line (NB, PL). Q.E.D.

Proof of Lemma 1.6: Consider a situation that $D_{P L}^{1}\left(p_{N B}^{1}, p_{P L}^{1}\right)=0$ in this case, and then $\lambda_{2} \geq 0$. Substituting the price $p_{N B}^{1}=p_{N B}^{*}$ and $p_{P L}^{1}=p_{P L}^{*}+\frac{\lambda_{1}}{2}\left(c_{P L, H}-c_{P L, L}\right)-\frac{\lambda_{2}}{2}$ into $D_{P L}^{1}\left(p_{N B}^{1}, p_{P L}^{1}\right)=0$ provides:

$$
\lambda_{2}=\left(c_{P L, H}-c_{P L, L}\right) \lambda_{1}-\frac{s_{P L, H}-s_{P L, L}}{s_{N B}-s_{P L, L}}\left(c_{N B}-c_{N B}^{c}\right) .
$$

If, on the one hand, $\lambda_{2}>0$, (A.1.13) implies: $\lambda_{1}>0$, which implies that the incentive compatibility constraint is binding. However, the incentive compatibility constraint in this case is

$$
\delta\left(p_{N B}^{2}-c_{N B}\right)\left(\bar{\theta}-\frac{p_{N B}^{2}-p_{P L}^{2}}{s_{N B}-s_{P L, H}}\right)>0,
$$

which is non-binding, thus it is a contradiction.

If, on the other hand, $\lambda_{2}=0$, (A.1.13) implies that $\lambda_{1}>0$ again, which implies that the incentive compatibility constraint is binding. However, similar to the previous situation, the constraint is non-binding, thus it is a contradiction.

As a result, the quantity of the PL product in this case cannot be zero in period 1. Q.E.D.

Proof of Lemma 1.7: For positive quantity of the PL product (see Lemma 1.6), the second constraint in the associated profit maximization problem is non-binding, i.e., $\lambda_{2}=0$. 
If the incentive compatibility constraint is binding, $\lambda_{1} \geq 0$, plugging $p_{N B}^{1}$ and $p_{P L}^{1}$ into the (binding) constraint, we can solve for the expression of $\lambda_{1}$ is:

$$
\begin{aligned}
\hat{\hat{\lambda}}_{1}= & \frac{s_{P L, H}-s_{P L, L}}{\left(s_{N B}-s_{P L, L}\right)\left(c_{P L, H}-c_{P L, L}\right)}\left(c_{N B}-c_{N B}^{c}\right) \\
& -\frac{2\left(s_{N B}-s_{P L, H}\right)\left(s_{P L, H}-s_{P L, L}\right)}{\left(s_{N B}-s_{P L, L}\right)\left(c_{P L, H}-c_{P L, L}\right)^{2}} \Pi_{N B, P L}^{2 *} .
\end{aligned}
$$

Note that we add "double-hat" on the $\lambda_{1}$ in this case in order to distinguish from the one in case $2(\mathrm{~A})$. In the meanwhile, plugging the prices into $D_{N B}^{1}\left(p_{N B}^{1}, p_{P L}^{1}\right)>0$ gives:

$$
\hat{\hat{\lambda}}_{1}<\frac{c_{N B}-c_{N B}^{c}}{c_{P L, H}-c_{P L, L}} \frac{s_{P L, H}-s_{P L, L}}{s_{N B}-s_{P L, L}} .
$$

With the help of the expression of $\hat{\hat{\lambda}}_{1}$, we can find that the inequality in (A.1.14) is satisfied since the second term in $\hat{\hat{\lambda}}_{1}$ is strictly positive. Moreover, we also require that $\hat{\hat{\lambda}}_{1} \geq 0$, which can be written as:

$$
\Lambda\left(c_{N B}\right)=\delta\left(c_{N B}^{a}-c_{N B}\right)\left(c_{N B}^{b}-c_{N B}\right)+(2+\delta)\left(c_{N B}-c_{N B}^{c}\right)\left(c_{P L, H}^{b}-c_{P L, H}\right) \leq 0 .
$$

We have:

$$
\left.\Lambda^{\prime}\left(c_{N B}\right)\right|_{c_{N B}=c_{N B}^{c}}<0, \quad \text { and }\left.\quad \Lambda^{\prime}\left(c_{N B}\right)\right|_{c_{N B}=c_{N B}^{b}}=0
$$

and

$$
\Lambda^{\prime \prime}\left(c_{N B}\right)=2 \delta>0
$$

(A.1.16) and (A.1.17) imply that the function $\Lambda\left(c_{N B}\right)$ is convex in $c_{N B}$ and monotonically decreasing in $c_{N B} \in\left(c_{N B}^{c}, c_{N B}^{b}\right)$. Further, the inequality in (A.1.15) can be satisfied if $c_{P L, H} \geq c_{P L, H}^{b}$ since the first term in this inequality is positive and $c_{N B}>c_{N B}^{c}$ in this case. Accordingly, we can conclude the first term is positive and the second term is negative. And $\Lambda\left(c_{N B}^{c}\right)=\delta\left(c_{N B}^{a}-c_{N B}^{c}\right)\left(c_{N B}^{b}-c_{N B}^{c}\right)>0$, 
$\Lambda\left(c_{N B}^{b}\right)=(2+\delta)\left(c_{N B}^{b}-c_{N B}^{c}\right)\left(c_{P L, H}^{b}-c_{P L, H}\right)<0$. By the continuity and monotonicity, we can conclude that there exists a unique value of $c_{N B}=c_{N B}^{e}$ such that $\Lambda\left(c_{N B}^{e}\right)=0$. In addition, $\Lambda\left(c_{N B}^{c} \leq c_{N B}<c_{N B}^{e}\right)>0$ and $\Lambda\left(c_{N B}^{e} \leq c_{N B}<c_{N B}^{b}\right)<0$. Therefore, in this case, the equilibrium with (NB, PL) exists if $c_{P L, H}^{b} \leq c_{P L, H}<c_{P L, H}^{a}$ and $c_{N B}^{e} \leq c_{N B}<c_{N B}^{b}$. And the equilibrium profit from (NB, PL) is $\hat{\Pi}_{N B, P L}\left(\hat{\hat{\lambda}}_{1}\right)$.

On the other hand, if the incentive compatibility constraint is non-binding, then $\hat{\hat{\lambda}}_{1}=0$, which implies that the retail prices on both products are at the benchmark level. Substituting these prices into the (non-binding) incentive compatibility constraint yields $\Lambda\left(c_{N B}>0\right.$. From (A.1.15), if $c_{P L, H}<c_{P L, H}^{b}$ this condition always holds. If $c_{P L, H} \geq c_{P L, H}^{b}$, this condition holds if $c_{N B}^{c}<c_{N B}<c_{N B}^{e}$. Therefore, the equilibrium profit from (NB, PL) is same as the one in case 3, i.e., $\Pi_{N B, P L}^{*}$. Q.E.D.

Proof of Proposition 1.6: Given the first scenario in Lemma 1.7, the joint profit from (NB, PL) is $\hat{\hat{\Pi}}_{N B, P L}$ and the retailer's bargaining profit is $\gamma \hat{\Pi}_{N B, P L}+(1-$ $\gamma) \hat{\Pi}_{P L}$. For simplicity in the following computation, we re-write this bargaining profit as:

$$
\gamma\left\{\left[\hat{\Pi}_{N B, P L}^{1}-\hat{\Pi}_{P L}^{1}\right]+\left[\Pi_{N B, P L}^{2 *}-\Pi_{P L}^{2 *}\right]\right\}+\hat{\Pi}_{P L}-K
$$

If the product line $(\mathrm{NB}, \mathrm{PL})$ is more profitable than $(0, \mathrm{PL})$, we have:

$$
\left[\hat{\hat{\Pi}}_{N B, P L}^{1}-\hat{\Pi}_{P L}^{1}\right]+\left[\Pi_{N B, P L}^{2 *}-\Pi_{P L}^{2 *}\right] \geq 0 .
$$

The second bracket in the left hand side from the above inequality is positive (due to Proposition 1.3). And in terms of the first, we have

$$
\hat{\hat{\Pi}}_{N B, P L}^{1}-\left.\hat{\Pi}_{P L}^{1}\right|_{c_{N B}=c_{N B}^{c}}>0,
$$

and

$$
\hat{\hat{\Pi}}_{N B, P L}^{1}-\left.\hat{\Pi}_{P L}^{1}\right|_{c_{N B}=c_{N B}^{b}}>0 .
$$


That implies even $\hat{\hat{\Pi}}_{N B, P L}^{1}$ is decreasing in $c_{N B}$, the value of $\hat{\hat{\Pi}}_{N B, P L}^{1}-\hat{\Pi}_{P L}^{1}$ stays positive for $c_{N B} \in\left(c_{N B}^{e}, c_{N B}^{b}\right)$, which implies that the product line (NB, PL) is more profitable than $(0, \mathrm{PL})$.

On the other hand, if the product line $(\mathrm{NB}, \mathrm{PL})$ is more profitable than $(\mathrm{NB}, 0)$, we have:

$$
\gamma\left[\left(\hat{\Pi}_{N B, P L}^{1}-\hat{\Pi}_{N B}^{1 *}\right)+\left(\Pi_{N B, P L}^{2 *}-\Pi_{N B}^{2 *}\right)\right]+(1-\gamma) \Pi_{P L}-K \geq 0 .
$$

The value of $(1-\gamma) \Pi_{P L}-K$ is positive for a very small $K$, and the value of $\prod_{N B, P L}^{2 *}-\Pi_{N B}^{2 *}$ is also positive. In terms of $\hat{\Pi}_{N B, P L}^{1}-\hat{\Pi}_{N B}^{1 *}$,

$$
\hat{\hat{\Pi}}_{N B, P L}^{1}-\left.\hat{\Pi}_{N B}^{1 *}\right|_{c_{N B}=c_{N B}^{e}}>0
$$

and

$$
\hat{\hat{\Pi}}_{N B, P L}^{1}-\left.\hat{\Pi}_{N B}^{1 *}\right|_{c_{N B}=c_{N B}^{b}}>0 .
$$

That implies the value of $\hat{\Pi}_{N B, P L}^{1}-\hat{\Pi}_{N B}^{1 *}$ is always positive for $c_{N B} \in\left(c_{N B}^{e}, c_{N B}^{b}\right)$ since $\Pi_{N B}^{1 *}$ is decreasing in $c_{N B} \in\left(c_{N B}^{e}, c_{N B}^{b}\right)$, which implies the product line (NB, PL) is more profitable than $(\mathrm{NB}, 0)$.

Given the second scenario in Lemma 1.7, the joint profit from (NB, PL) is same as the one under perfect information, i.e., $\Pi_{N B, P L}^{*}$. In Proposition 1.3, we have proved that the product line (NB, PL) with this joint profit is the most profitable one and the retailer will choose it in equilibrium. Q.E.D.

Proof of Proposition 1.7: In case of $c_{N B}^{b} \leq c_{N B}<c_{N B}^{d}$ and $c_{P L, H}^{b} \leq c_{P L, H}<c_{P L, H}^{a}$, The difference between these two consumer surplus is:

$$
\begin{aligned}
C S_{N B, P L}-C S_{P L}= & \frac{s_{N B}}{2} D_{N B}^{1}\left(\hat{\lambda}_{1}\right)-p_{N B} D_{N B}^{1}\left(\hat{\lambda}_{1}\right)+\frac{s_{P L, H}}{2}\left[D_{P L}^{1}\left(\hat{\lambda}_{1}\right)+D_{P L}^{1}(\hat{\lambda})\right]\left[D_{P L}^{1}\left(\hat{\lambda}_{1}\right)-D_{P L}^{1}(\hat{\lambda})\right] \\
& \left.\left.+p_{P L}^{1}(\hat{\lambda})\right) D_{P L}^{1}(\hat{\lambda})-p_{P L}^{1}(\hat{\lambda})_{1}\right) D_{P L}^{1}\left(\hat{\lambda}_{1}\right) .
\end{aligned}
$$


In addition, we can find that:

$$
\left.\left[D_{P L}^{1}\left(\hat{\lambda}_{1}\right)-D_{P L}^{1}(\hat{\lambda})\right]\right|_{c_{N B}=c_{N B}^{b}}=0
$$

and

$$
\left.\left[D_{P L}^{1}\left(\hat{\lambda}_{1}\right)-D_{P L}^{1}(\hat{\lambda})\right]\right|_{c_{N B}=c_{N B}^{d}}>0
$$

which implies that the second term of $C S_{N B, P L}-C S_{P L}$ is positive. Moreover,

$$
\left.\left.p_{P L}^{1}(\hat{\lambda})\right) D_{P L}^{1}(\hat{\lambda})-p_{P L}^{1}(\hat{\lambda})_{1}\right)\left.D_{P L}^{1}\left(\hat{\lambda}_{1}\right)\right|_{c_{N B}=c_{N B}^{b}}>0
$$

which implies that the last term of $C S_{N B, P L}-C S_{P L}$ is also positive. Overall, the consumer surplus is greater in the product line (NB, PL). Q.E.D. 


\section{Appendix B}

\section{Appendix for Chapter 2}

Proof of Lemma 2.1: Given the prices in (2.7), we discuss different cases according to the constraints in the problem (2.5).

Case 1: If $D_{N B}^{1}>0, D_{N B}^{t \geq 2}>0, D_{P L}^{1}>0$ and $D_{P L}^{t}=0$. The retailer drops the PL product in period 2. The prices are therefore $p_{N B}^{1}=p_{N B}^{t}=p_{N B}^{*}, p_{P L}^{1}=p_{P L}^{*}$ and $p_{P L}^{t}=p_{P L}^{*}-\mu_{2}^{t} / 2$. Given that $D_{P L}^{1}>0$ and $p_{P L}^{t} \leq p_{P L}^{1}, D_{P L}^{t}$ should satisfy that $D_{P L}^{t} \geq D_{P L}^{1}>0$, which is a contradiction.

Case 2: If $D_{N B}^{1}>0, D_{N B}^{t \geq 2}>0, D_{P L}^{1}=0$ and $D_{P L}^{t}>0$. The retailer adds the PL product in period 2. The prices are therefore $p_{N B}^{1}=p_{N B}^{t}=p_{N B}^{*}, p_{P L}^{1}=p_{P L}^{*}-\mu_{2}^{1} / 2$ and $p_{P L}^{t}=p_{P L}^{*}$. Given that $D_{P L}^{1}=0$ and $p_{P L}^{1} \leq p_{P L}^{t}, D_{P L}^{t}$ should satisfy that $D_{P L}^{t} \geq D_{P L}^{1}=0$, which is a contradiction.

Case 3: If $D_{N B}^{1}>0, D_{N B}^{t \geq 2}=0, D_{P L}^{1}>0$ and $D_{P L}^{t}>0$. The retailer drops the NB product in period 2. The prices are therefore $p_{N B}^{1}=p_{N B}^{*}, p_{N B}^{t}=p_{N B}^{*}-\mu_{1}^{t} / 2$ and $p_{P L}^{1}=p_{P L}^{t}=p_{P L}^{*}$. Given that $D_{N B}^{t \geq 2}=0$ and $p_{N B}^{t} \leq p_{N B}^{1}, D_{N B}^{1}$ should satisfy that $D_{N B}^{1} \geq D_{N B}^{t \geq 2}=0$, which is a contradiction.

Case 4: If $D_{N B}^{1}=0, D_{N B}^{t \geq 2}>0, D_{P L}^{1}>0$ and $D_{P L}^{t}>0$. The retailer adds the NB product in period 2. The prices are therefore $p_{N B}^{1}=p_{N B}^{*}-\mu_{1}^{1} / 2, p_{N B}^{t}=p_{N B}^{*}$ and $p_{P L}^{1}=p_{P L}^{t}=p_{P L}^{*}$. Given that $D_{N B}^{1}=0$ and $p_{N B}^{1} \leq p_{N B}^{t}, D_{N B}^{t \geq 2}$ should satisfy that 
$D_{N B}^{t \geq 2} \leq D_{N B}^{1}=0$, which is a contradiction.

Case 5: If $D_{N B}^{1}>0, D_{N B}^{t \geq 2}=0, D_{P L}^{1}=0$ and $D_{P L}^{t}>0$. The retailer replaces the NB product by the PL product in period 2. The prices are therefore $p_{N B}^{1}=p_{N B}^{*}, p_{N B}^{t}=$ $p_{N B}^{*}-\mu_{1}^{t} / 2$ and $p_{P L}^{1}=p_{P L}^{*}-\mu_{2}^{1} / 2$ and $p_{P L}^{t}=p_{P L}^{*}$. Given that $p_{N B}^{1} \geq p_{N B}^{t}$ and $p_{P L}^{1} \leq p_{P L}^{t}$, dnbfst and $D_{P L}^{1}$ should satisfy that $D_{N B}^{1} \leq D_{N B}^{t \geq 2}=0$ and $D_{P L}^{1} \geq D_{P L}^{t}>0$, which is a contradiction.

Case 6: If $D_{N B}^{1}=0, D_{N B}^{t \geq 2}>0, D_{P L}^{1}>0$ and $D_{P L}^{t}=0$. The retailer replaces the PL product by the NB product in period 2. The prices are therefore $p_{N B}^{1}=$ $p_{N B}^{*}-\mu_{1}^{1} / 2, p_{N B}^{t}=p_{N B}^{*}$ and $p_{P L}^{1}=p_{P L}^{*}$ and $p_{P L}^{t}=p_{P L}^{*}-\mu_{2}^{t} / 2$. Given that $p_{N B}^{1} \leq p_{N B}^{t}$ and $p_{P L}^{t} \leq p_{P L}^{1}$, dnbfst and $D_{P L}^{1}$ should satisfy that $D_{N B}^{1} \geq D_{N B}^{t \geq 2}>0$ and $D_{P L}^{1} \leq D_{P L}^{t}=0$, which is a contradiction.

As a result, in the case where the negotiation between the two firms is successful, the retailer does not wish to change its product line in period 2. Q.E.D.

Proof of Lemma 2.2: In the profit maximization problem (2.5), if $D_{N B}^{1}=$ $D_{N B}^{t \geq 2}=0, D_{P L}^{1} \geq 0$ and $D_{P L}^{t}>0$. The retail prices are therefore $p_{N B}=p_{N B}^{*}-\mu_{1} / 2$ and $p_{P L}=p_{P L}^{*}$. Substituting these retail prices into the constraints, we can obtain

$$
D_{N B}=\frac{\bar{\theta}}{2}\left(s_{N B}-s_{P L, H}\right)-\frac{c_{N B}}{2}+\frac{c_{P L, H}}{2}+\frac{\mu_{1}}{2}=0,
$$

and

$$
D_{P L}=\frac{\bar{\theta}}{2}\left(s_{P L, H}-s_{P L, L}\right)-\frac{c_{P L, H}}{2}+\frac{c_{P L, L}}{2}>0 .
$$

(A.2.1) solves that $\mu_{1}=c_{N B}-\bar{\theta}\left(s_{N B}-s_{P L, H}\right)-c_{P L, H}$, and $\mu_{1} \geq 0$ requires that $c_{N B} \geq \bar{\theta}\left(s_{N B}-s_{P L, H}\right)+c_{P L, H}$, which is $c_{N B}^{b}$. Moreover, (A.2.2) shows that $c_{P L, H}<c_{P L, H}^{a}$, which is equivalent to Assumption 2.2. Q.E.D.

Proof of Proposition 2.1: Given Table 2.2 in the text, the retailers bargaining payoff from the product line (NB, PL) is $\Pi_{P L}^{*}-K$. The retailer's bargaining payoff 
from the product line $(\mathrm{NB}, 0)$ is $\gamma \Pi_{N B}^{*}+(1-\gamma)\left(\Pi_{P L}^{-1 *}-\delta K\right)$. Moreover, we have

$$
\begin{aligned}
\Pi_{P L}^{*}- & \Pi_{N B}^{*}=\frac{1+\delta}{1-\delta} \frac{1}{s_{P L, H}-s_{P L, L}}\left[\bar{\theta}\left(s_{P L, H}-s_{P L, L}\right)\right. \\
- & \left.\left(c_{P L, H}-c_{P L, L}\right)\right]^{2}-\frac{1+\delta}{1-\delta} \frac{1}{s_{N B}-s_{P L, L}}\left[\bar{\theta}\left(s_{N B}-s_{P L, L}\right)-\left(c_{N B}-c_{P L, L}\right)\right]^{2} \\
= & \frac{1+\delta}{1-\delta}\left\{\left[\bar{\theta}\left(s_{P L, H}-s_{P L, L}\right)-\left(c_{P L, H}-c_{P L, L}\right)\right]\left[\bar{\theta}-\frac{c_{P L, H}-c_{P L, L}}{s_{P L, H}-s_{P L, L}}\right]\right. \\
- & {\left.\left[\bar{\theta}\left(s_{N B}-s_{P L, L}\right)-\left(c_{N B}-c_{P L, L}\right)\right]\left[\bar{\theta}-\frac{c_{N B}-c_{P L, L}}{s_{N B}-s_{P L, L}}\right]\right\} \geq 0 . }
\end{aligned}
$$

This implies that $\Pi_{N B, P L}-\Pi_{N B}=\gamma\left(\Pi_{P L}^{*}-\Pi_{N B}^{*}\right)-(1-\gamma)\left(\Pi_{P L}^{*}-\Pi_{P L}^{-1 *}+\delta K\right)-K>0$ for a small $K$. As a result, the retailer will choose the product line $(0, \mathrm{PL})$ in equilibrium and, not have incentive to engage into a negotiation with the NB supplier. Q.E.D.

Proof of Lemma 2.3: Given that $p_{P L}^{1}=p_{P L}^{*}+\lambda\left(c_{P L, H}-c_{P L, L}\right) / 2$ and $p_{P L}^{t}=p_{P L}^{*}+$ $\lambda\left(c_{P L, H}-c_{P L, L}\right) / 2(2+\lambda)$. If the retailer's incentive compatibility constraint in $(2.10)$ is non-binding, the retail price will be identical to that under perfect information, i.e., $p_{P L}^{1}=p_{P L}^{t}=p_{P L}^{*}$. Substituting $p_{P L}^{*}$ into the incentive compatibility constraint yields

$$
\left(c_{P L, L}-c_{P L, H}\right) D_{P L}\left(p_{P L}^{*}\right)+\frac{\delta}{1-\delta}\left(p_{P L}^{*}-2 c_{P L, H}+c_{P L, L}\right) D_{P L}\left(D_{P L}^{*}\right)>0
$$

which implies that $c_{P L, H}<c_{P L, H}^{b}=\delta \theta\left(s_{P L, H}-s_{P L, L}\right) /(2+\delta)+c_{P L, L}$. In addition, the profit in this case is same as that under perfect information, i.e., $\Pi_{P L}=\Pi_{P L}^{*}$.

On the other hand, if the incentive compatibility constraint in (2.10) is binding, then the multiplier $\lambda$ is non-negative and can be determined by $\Pi\left(p_{P L}^{1}, p_{P L}^{t}\right)-$ $\widetilde{\Pi}\left(p_{P L}^{1}, p_{P L}^{t}\right)=0$. Note that this equation reveals that the punishment to the lowquality retailer is at a cut-off point, i.e., zero. For the computational simplicity, we consider a situation that the punishment in period 1 is zero. In fact, such punishment is a negative value because all consumers do not know the quality of the PL product and the retailer can obtain a strictly positive payoff from cheating on them. Accord- 
ingly, we can obtain the critical values on the marginal costs which are greater than the actual one by assuming the punishment in period 1 is zero. Following this line, we can write the binding incentive compatibility constraint in (2.10) as

$$
\left(\frac{\hat{\lambda}}{2+\hat{\lambda}}\right)^{2}-2\left(\frac{\hat{\lambda}}{2+\hat{\lambda}}\right)-A=0
$$

where

$$
A=\frac{\left[\bar{\theta}\left(s_{P L, H}-s_{P L, L}\right)-\left(c_{P L, H}-c_{P L, L}\right)\right]\left[\bar{\theta}\left(s_{P L, H}-s_{P L, L}\right)-3\left(c_{P L, H}-c_{P L, L}\right)\right]}{\left(c_{P L, H}-c_{P L, L}\right)^{2}} \leq 0
$$

for $c_{P L, H}^{b} \leq c_{P L, H}<c_{P L, H}^{a}$. Solving (A.2.3) yields

$$
\hat{\lambda}=\frac{2(1-\sqrt{1+A}}{\sqrt{1+A}} .
$$

Moreover, the retailer's profit from $(0, \mathrm{PL})$ is positive if

$$
\left(\frac{\hat{\lambda}}{2+\hat{\lambda}}\right)^{2}<\left[\frac{\bar{\theta}\left(s_{P L, H}-s_{P L, L}\right)-\left(c_{P L, H}-c_{P L, L}\right)}{c_{P L, H}-c_{P L, L}}\right]^{2} .
$$

With the expression of $\hat{\lambda},($ A.2.4) implies that

$$
\bar{\theta}\left(s_{P L, H}-s_{P L, L}\right) / 3+c_{P L, L} \leq c_{P L, H}<\bar{\theta}\left(s_{P L, H}-s_{P L, L}\right) / 2+c_{P L, L} .
$$

Therefore, there exists a $c_{P L, H}-c_{P L, H}$, which is smaller than $\bar{\theta}\left(s_{P L, H}-s_{P L, L}\right) / 2+c_{P L, L}$ that satisfies both (A.2.3) and (A.2.4). In addition, the retail prices on the PL product are increased by a term $\hat{\lambda}\left(c_{P L, H}\right)$ and, the retailer's profit is reduced to $\hat{\Pi}_{P L}>0$. If $c_{P L, H}$ is no smaller than this critical value, the retailer's profit will be non-positive.

Moreover, if the retailer chooses this product in period 2, then the profit maxi- 
mization problem will be modified to

$$
\begin{aligned}
& \max \Pi_{P L}\left(p_{P L}^{t}\right)=\sum_{t=2}^{\infty} 2 \delta^{t-1}\left(p_{P L}^{t}-c_{P L, H}\right) D_{P L}\left(p_{P L}^{t}\right) \\
& \text { s.t. : } \Pi_{P L}\left(p_{P L}^{t}\right) \geq \widetilde{\Pi}_{P L}\left(p_{P L}^{t}\right),
\end{aligned}
$$

where $\widetilde{\Pi}_{P L}\left(p_{P L}^{t}\right)=\sum_{t=2}^{\infty} \delta^{t-1}\left(p_{P L}^{t}-c_{P L, L}\right) D_{P L}\left(p_{P L}^{t}\right)$. By applying the same method as the above proof, the equilibrium profit in this case is $\Pi_{P L}^{*}$ less the first-period profit if $c_{P L, H}<c_{P L, H}^{b}$, which is denoted by $\Pi_{P L}^{-1 *}$. If $c_{P L, H}^{b} \leq c_{P L, H}<\bar{\theta}\left(s_{P L, H}-s_{P L, L}\right) / 2+c_{P L, L}$, the equilibrium profit is $\hat{\Pi}_{P L}$ less the first-period profit, denoted by $\hat{\Pi}_{P L}^{-1}$. The equilibrium profit is zero if $\bar{\theta}\left(s_{P L, H}-s_{P L, L}\right) / 2+c_{P L, L} \leq c_{P L, H}<c_{P L, H}^{a}$. Q.E.D.

Proof of Lemma 2.4: In the problem (2.14), if the retailer's incentive compatibility constraint is non-binding, i.e., if $\nu=0$ and the price of the PL product is $p_{P L}^{*}$ in every period. In this case, the equilibrium demand for the NB product cannot be positive in any period. For instance, if the demand for the NB product is positive in period $t, t \geq 1$, then $\xi=0$ in such period, which causes that the price on the NB product to be identical to $p_{N B}^{*}$. Given the hold of Lemma 2.2, i.e., $c_{N B} \geq c_{N B}^{b}$, the demand cannot be positive with retail prices $\left(p_{N B}^{*}, p_{P L}^{*}\right)$. As a result, the demand for the NB product can only be zero if the incentive compatibility constraint is non-binding. In addition, this situation is same as that in Lemma 2.3.

Moreover, if the retailer's incentive compatibility constraint is binding, i.e., if $\xi \geq 0$, the retail price on the PL product will be increased by a term of $\nu$. Consider the case that $D_{N B}^{1}=0$ and $D_{N B}^{t \geq 2}>0$, i.e., $\xi_{1} \geq 0$ and $\xi_{2}=0$. Then $D_{N B}^{1}=0$ and $D_{N B}^{t \geq 2}>0$ respectively show that

$$
\xi_{1}+\nu\left(c_{P L, H}-c_{P L, L}\right)=c_{N B}-c_{N B}^{b}
$$


and

$$
-\xi_{1}-\nu\left(c_{P L, H}-c_{P L, L}\right)+\frac{\nu}{2+\nu}\left(c_{P L, H}-c_{P L, L}\right)>0 .
$$

Note that condition (A.2.6) cannot hold since $\nu>\nu /(2+\nu)$. Therefore, it is not possible that the demand for the NB product is zero in period 1 and positive in period 2 .

Consider the case, on the other hand, that $D_{N B}^{1}>0$ and $D_{N B}^{t \geq 2}=0$, i.e., $\xi_{1}=0$ and $\xi_{2} \geq 0$. Then $D_{N B}^{1}>0$ and $D_{N B}^{t \geq 2}=0$ respectively show that

$$
\nu>\frac{c_{N B}=c_{N B}^{b}}{c_{P L, H}-c_{P L, L}},
$$

and

$$
\xi_{2}=(2+\nu)\left(c_{N B}-c_{N B}^{b}\right)-\nu\left(c_{P L, H}-c_{P L, L}\right) .
$$

It is possible that both (A.2.7) and (A.2.8) hold at the same time.

Finally, if $D_{N B}^{1}=D_{N B}^{t \geq 2}=0$, i.e., $\xi \geq 0$ and $\xi_{2} \geq 0$, then the case will be identical to that of $(0, \mathrm{PL})$ with binding incentive compatibility, and the equilibrium will be same to that in Lemma 2.3. Q.E.D.

Proof of Lemma 2.5: In case where the demand for the NB product is positive in every period, $\xi_{1}=\xi_{2}=0$. Here, we still follow the method in the proof of Lemma 2.3 , i.e., assume that the punishment to the retailer is zero in period 1 . The binding incentive compatibility in the problem (2.14) shows that

$$
\left(\frac{\hat{\lambda}}{2+\hat{\lambda}}\right)^{2}-2\left(\frac{\hat{\lambda}}{2+\hat{\lambda}}\right)-B=0,
$$


where

$$
\begin{aligned}
B= & \frac{\left[\left(c_{N B}\left(s_{P L, H}-s_{P L, L}\right)-c_{P L, H}\left(s_{N B}-s_{P L, L}\right)+c_{P L, L}\left(s_{N B}-s_{P L, H}\right)\right]\right.}{\left(s_{N B}-s_{P L, L}\right)\left(s_{P L, H}-c_{P L, L}\right)^{2}} \\
& \times\left[\bar{\theta}\left(s_{P L, H}-s_{P L, L}\right)-3\left(c_{P L, H}-c_{P L, L}\right)\right] .
\end{aligned}
$$

In addition, the demand for the NB product is positive requires that

$$
\frac{\hat{\lambda}}{2+\hat{\lambda}}>\frac{c_{N B}-c_{N B}^{b}}{c_{P L, H}-c_{P L, L}}
$$

The condition (A.2.10) solves that $c_{N B}^{b} \leq c_{N B}<\bar{\theta}\left(s_{N B}-2 s_{P L, H}+s_{P L, L}\right)+4 c_{P L, H}-3 c_{P L, L}$, which implies the actual critical value on $c_{N B}$, i.e., $c_{N B}=c_{N B}^{c}$, exists and smaller than $\bar{\theta}\left(s_{N B}-2 s_{P L, H}+s_{P L, L}\right)+4 c_{P L, H}-3 c_{P L, L}$ (but still greater than $c_{N B}^{b}$ if $c_{P L, H}^{b} \leq c_{P L, H}<$ $\left(c_{P L, H}^{c}\right)$.

On the other hand, if the demand for the NB product is positive in period 1 only, $\xi_{1}=0$ and $\xi_{2} \geq 0$. Moreover, $\hat{\xi}_{2}=(2+\hat{\nu})\left(c_{N B}-c_{N B}^{b}\right)-\hat{\nu}\left(c_{P L, H}-c_{P L, L}\right) \geq 0$, which is oppose to the condition (A.2.10). The binding incentive compatibility constraint in the problem (2.14) shows that

$$
\left(\frac{\hat{\nu}}{2+\hat{\nu}}\right)^{2}-2\left(\frac{\hat{\nu}}{2+\hat{\nu}}-\Gamma=0,\right.
$$

where

$$
\Gamma=\frac{\left[\bar{\theta}\left(s_{P L, H}-s_{P L, L}\right)-\left(c_{P L, H}-c_{P L, L}\right)\right]\left[\bar{\theta}\left(s_{P L, H}-s_{P L, L}\right)-3\left(c_{P L, H}-c_{P L, L}\right)\right]}{\left(s_{N B}-s_{P L, L}\right)\left(c_{P L, H}-c_{P L, L}\right)^{2}} .
$$

The condition (A.2.11) can solve that

$$
\hat{\nu}=\frac{2(1-\sqrt{1+\Gamma}}{\sqrt{1+\Gamma}}
$$


And for non-negative $\hat{\xi}_{2}$, we have $\bar{\theta}\left(s_{N B}-2 s_{P L, H}+s_{P L, L}\right)+4 c_{P L, H}-3 c_{P L, L} \leq c_{N B}<c_{N B}^{a}$, which implies that $c_{N B}$ is no smaller than the critical value in the previous case. Further, the upper-bound on $c_{N B}$ in this case is $c_{N B}^{a}$. Because we have assumed that the punishment to the retailer is zero in period 1, the actual upper-bound is smaller than $c_{N B}^{a}$. Consequently, there exists a range on $c_{N B}$, say $c_{N B}^{d} \leq c_{N B}<c_{N B}^{a}$, such that $\hat{\nu}$ is negative. In addition, the set $\left(c_{N B}^{d}, c_{N B}^{a}\right)$ is non-empty for non-zero punishment to the retailer in period 1 if $c_{P L, H}^{b} \leq c_{P L, H}<c_{P L, H}^{a}$.

Furthermore, it can be easily found that $\Gamma<A$, which implies that $\hat{\nu}<\hat{\lambda}$. The retail prices of the PL product in the product line $(0, \mathrm{PL})$ and $(\mathrm{NB}, \mathrm{PL})$ are monotonic (e.g., increasing) function about $\hat{\lambda}$ and $\hat{\nu}$, respectively. Given $\hat{\lambda}>\hat{\nu}$, the price of the PL product in the product line $(0, \mathrm{PL})$ is greater than that in $(\mathrm{NB}, \mathrm{PL})$. Q.E.D.

Proof of Proposition 2.2: If $c_{P L, H}<c_{P L, H}^{b}$, the retailer sells only the PL product in sub-game equilibrium and obtains the first-best profit $\Pi_{P L}^{*}$ which is greater than that from selling only the NB product (see the proof of Proposition 2.1).

If $c_{P L, H}^{b} \leq c_{P L, H}<c_{P L, H}^{c}$ and $c_{N B}^{b} \leq c_{N B}<c_{N B}^{c}$, the retailer sells both products in every period, and obtains a bargaining profit $\gamma \hat{\hat{\Pi}}_{N B, P L}+(1-\gamma) \hat{\Pi}_{P L}$ with this diversified product line. If the product line $(\mathrm{NB}, \mathrm{PL})$ is profitable relative to $(0, \mathrm{PL})$, we have

$$
\gamma\left(\hat{\hat{\Pi}}_{N B, P L}^{1}+\frac{\delta}{1-\delta} \hat{\Pi}_{N B, P L}^{t}\right)+(1-\gamma)\left(\hat{\Pi}_{P L}^{1}+\frac{\delta}{1-\delta} \hat{\Pi}_{P L}^{t}\right) \geq \hat{\Pi}_{P L}^{1}+\frac{\delta}{1-\delta} \hat{\Pi}_{P L}^{t},
$$

which is

$$
\hat{\hat{\Pi}}_{N B, P L}^{1}-\hat{\Pi}_{P L}^{1}+\frac{\delta}{1-\delta}\left(\hat{\Pi}_{N B, P L}^{t}-\hat{\Pi}_{P L}^{t}\right) \geq 0 .
$$

The associate profits can be expressed as

$$
\hat{\hat{\Pi}}_{N B, P L}=\Pi_{N B, P L}^{*}-f(\hat{\nu}),
$$


and

$$
\hat{\Pi}_{P L}=\Pi_{P L}^{*}-f(\hat{\lambda})
$$

In addition, $f^{\prime}(\cdot) \dot{i} 0$. It can be found that $\hat{\nu} \leq \hat{\lambda}$ (see the proof of Lemma 2.5). Therefore, the condition (A.2.12) can be written as

$$
\left(\Pi_{N B, P L}^{*}-\Pi_{P L}^{*}\right)+[f(\hat{\lambda})-f(\hat{\nu})] \geq 0,
$$

which is satisfied. Moreover, if the product line (NB, PL) is more profitable than $(\mathrm{NB}, 0)$, then we have

$$
\gamma\left(\hat{\hat{\Pi}}_{N B, P L}-\Pi_{N B}^{*}\right)+(1-\gamma)\left(\hat{\Pi}_{P L}-\hat{\Pi}_{P L}^{-1}\right)-(1-\delta) K \geq 0 .
$$

The first term is non-negative for $c_{N B}^{b} \leq c_{N B}<c_{N B}^{c}$, and the second term is positive for sure. Therefore, the above inequality holds for a small $K$.

If $c_{P L, H}^{b} \leq c_{P L, H}<c_{P L, H}^{c}$ and $c_{N B}^{c} \leq c_{N B}<c_{N B}^{d}$, the retailer sells both products in period 1 only, and obtains a bargaining profit $\gamma \hat{\hat{\Pi}}_{N B, P L}+(1-\gamma) \hat{\Pi}_{P L}$. If the product line $(\mathrm{NB}, \mathrm{PL})$ is profitable relative to $(0, \mathrm{PL})$, we have

$$
\gamma\left(\hat{\hat{\Pi}}_{N B, P L}^{1}+\frac{\delta}{1-\delta} \hat{\Pi}_{P L}^{t}\right)+(1-\gamma)\left(\hat{\Pi}_{P L}^{1}+\frac{\delta}{1-\delta} \hat{\Pi}_{P L}^{t}\right) \geq \hat{\Pi}_{P L}^{1}+\frac{\delta}{1-\delta} \hat{\Pi}_{P L}^{t},
$$

which is

$$
\hat{\hat{\Pi}}_{N B, P L}^{1} \geq \hat{\Pi}_{P L}^{1}
$$

With the result in the previous case, condition (A.2.13) holds. If the product line $(\mathrm{NB}, \mathrm{PL})$ is more profitable than $(\mathrm{NB}, 0)$, then we have

$$
\left(\hat{\hat{\Pi}}_{N B, P L}-\Pi_{N B}^{*}\right)+(1-\gamma)\left(\hat{\Pi}_{P L}-\hat{\Pi}_{P L}^{-1}\right)-(1-\delta) K \geq 0 .
$$


The first term is non-negative for $c_{N B}^{c} \leq c_{N B}<c_{N B}^{d}$, and the second term is positive for sure. Therefore, the above inequality holds for a small $K$.

$$
\text { If } c_{P L, H}^{b} \leq c_{P L, H}<c_{P L, H}^{c} \text { and } c_{N B}^{d} \leq c_{N B}<c_{N B}^{a} \text {, offering the product line (NB, PL) }
$$
will not be an equilibrium. The retailer will then sell either only NB product or only $\mathrm{PL}$ product. If the product line $(0, \mathrm{PL})$ is more profitable than $(\mathrm{NB}, 0)$, then we have

$$
\left(\hat{\Pi}_{P L}-\Pi_{N B}^{*}\right)+(1-\gamma)\left(\hat{\Pi}_{P L}-\hat{\Pi}_{P L}^{-1}+\delta K\right)-K \geq 0
$$

The first term is non-negative for $c_{P L, H}^{b} \leq c_{P L, H}<c_{P L, H}^{c}$, and the second term is positive for sure. Therefore, the above inequality holds for a small $K$.

If $c_{P L, H}^{c} \leq c_{P L, H}<c_{P L, H}^{a}$, the cost of the PL product is so high, and offering the product line that contains the PL product will not be an equilibrium. As a result, the retailer will sell only the NB product in equilibrium. Q.E.D. 


\section{Appendix C}

\section{Appendix for Chapter 3}

Derivation of the pass-through term: $\frac{\partial p_{j}}{\partial w_{i}}$.

For a given brand $l \in J$, totally differentiating the retailer's associate first-order condition (equation (3.10) in the text) with respect to wholesale price $w_{m}$ and $m \in J$, we can obtain

$$
\left[s_{l m}+s_{m l}+\sum_{j=1}^{J}\left(\left(p_{z j t}-w_{j z t}\right) \cdot \frac{\partial s_{j m}}{\partial p_{l}}\right)\right] d p_{l}-s_{l m} d w_{m}=0
$$

which implies

$$
g_{l m}=\frac{d p_{l}}{d w_{m}}=s_{l m}+s_{m l}+\sum_{j=1}^{J}\left(p_{j}-w_{j}\right) \frac{\partial s_{j m}}{\partial p_{l}},
$$

where $s_{l m}=\frac{\partial s_{l}}{\partial p_{m}}$. In addition, $g_{l m}$ represents the element in row $l$ and column $m$ of the pass-through matrix $G$. Further, since both $l$ and $m$ are row-column-specific whereas $j$ is not, the value of twice differentiation $\frac{\partial s_{j m}}{\partial p_{l}}$ relies on the value of $l, m$ and $j$. More specifically, the value of $\frac{\partial s_{j m}}{\partial p_{l}}$ has five possibilities as following:

1. if $j=m=l, \frac{\partial s_{j m}}{\partial p_{l}}=\alpha^{2} s_{j}\left(1-s_{j}\right)\left(1-2 s_{j}\right)$

2. if $j \neq m \neq j, \frac{\partial s_{j m}}{\partial p_{l}}=2 \alpha^{2} s_{j} s_{m} s_{l}$ 
3. if $j \neq m=l, \frac{\partial s_{j m}}{\partial p_{l}}=\alpha^{2} s_{m} s_{j}\left(2 s_{m}-1\right)$

4. if $j=m \neq l, \frac{\partial s_{j m}}{\partial p_{l}}=\alpha^{2} s_{j} s_{l}\left(2 s_{j}-1\right)$

5. if $j=l \neq m, \frac{\partial s_{j m}}{\partial p_{l}}=\alpha^{2} s_{j} s_{m}\left(2 s_{j}-1\right)$

In programming of manufacturer' profit-maximizing margins, we will take these five possibilities into account. 